\title{
Unravelling the Role Cortical Myelination Plays in Higher Brain Functions
}

\author{
Dissertation \\ for the award of the degree \\ “Doctor rerum naturalium” (Dr. rer. nat.) \\ of the Georg-August-Universität Göttingen
}

within the IMPRS Molecular Biology

of the Georg-August University School of Science (GAUSS)

submitted by

Swati Subramanian

from New Delhi, India

Göttingen, 2020 


\section{Examination Board}

\section{Thesis Committee}

Dr. Livia de Hoz (Supervisor)

Department of Neurogenetics, Max Planck Institute for Experimental Medicine

Prof. Dr. Klaus-Armin Nave ( $1^{\text {st }}$ Reviewer)

Department of Neurogenetics, Max Planck Institute for Experimental Medicine

Prof. Dr. Silvio Rizzoli (2 ${ }^{\text {nd }}$ Reviewer)

Department of Neuro- and Sensory Physiology, University Medical Center Göttingen

Prof. Dr. Nils Brose

Department of Molecular Neurobiology, Max Planck Institute for Experimental Medicine

\section{Extended Examination Board}

Prof. Dr. Tobias Moser

University Medical Center Göttingen

Institute for Auditory Neuroscience \& InnerEar Lab

Dr. Alexander Stein

Membrane Protein Biochemistry, Max Planck Institute for Biophysical Chemistry

Dr. Melina Schuh

Department of Meiosis, Max Planck Institute for Biophysical Chemistry

Date of oral examination: 09.02.2021 


\section{Declaration}

I hereby declare that the PhD thesis entitled, "Unravelling the Role Cortical Myelination Plays in Higher Brain Functions", was written independently and with no other sources and aids than quoted.

Göttingen, 14.12.2020 


\section{Acknowledgments}

I would like to begin with a big thank you to Dr. Livia de Hoz, my direct supervisor. You were a constant source of support, feedback and taught me how to hone my critical thinking. Thank you for believing in my capabilities and me, even when I had doubts myself. Thanks to Prof. Klaus-Armin Nave for bringing me in on this very interesting project, for giving me support when I needed it and for being the ultimate devil's advocate, challenging me to learn more and beyond the project itself.

Thank you to my thesis advisory committee members, Prof. Silvio Rizzoli and Prof. Nils Brose. Your genuine interest in the project and the feedback was of essence in making this thesis what it is. This project would not have been possible without the contributions of Dr. Wiebke Möbius, Torben Ruhwedel and Boguslawa Sadowski who provided me the mouse lines, their expertise and help with EM. A special thanks to Jonas Hemesath for being a crucial part of the EMX and FOXG1 projects and for his help in their characterisation.

I want to thank Dr. Sharlen Moore, Dr. Chi Chen and Philipp van Kronenberg for making the first two years of the $\mathrm{PhD}$ filled with lively discussions be it about science or food. You are all dearly missed in the lab.

Other members of the lab include, Maria Eichel, Constanze Depp, Ting Sun, Stefan Berghoff, Andrew Octavian Sasmita, Tobias Buscham, Lena Spieth, Lisa Linhoff, Silya Garagareta, Sophie Siems and Ramona Jung. Thank you all for being someone I could always count on for coffee, cakes and conversation.

Maria, Constanze, Ting, Stefan and Andrew in particular. Our friendship goes beyond the lab and I am truly thankful that I got know each and every one of you. Big thanks to the animal facility members, Cornelia Casper and Tanja Hoffmeister for taking such good care of my mice.

A big thank you to Michaela Schmalstieg for helping me manoeuvre the German administrative process and the fun chats with coffee.

To my friends outside the lab, thank you Valentina Manzini, Rashi Goel, Laura Arranz, Salma Sohrabi-Jahromi and Linda Olsthoorn for being there for the best and the worst times.

Thank you to Dr. Steffen Burkhardt and Kerstin Grüniger. Your help in making sure that the transition, of an international student like me, into the daily university life 
was seamless and Steffen, I appreciated all the support, advice and discussions we've had over the years.

Danke Niklas Michel, Karin Michel, Gregor Michel, Markus Reis und dem Rest der Familie, dass sie für so viele Jahre meine zweite Familie waren. Ich schätze jeden Moment, den ich in eurer Gesellschaft verbracht habe.

I want to thank my friends back home, Sonali Chowdhry, Madhobi Sen, Vaishnavi Varadarajan, Rohini Subrahmanyam, Ruchi Hotchandani and Sumedha Anand. You women are, and have always been my rock. Thank you for being there through thick and thin.

Last but by far not the least I want to thank my mother, Santhi Subramanian and my late father, K. Subramanian for making me the person I am today, for teaching me the value of good education and the importance of being a good human. Dad, I miss you every day and I wish you were here to see this day. 
"Be less curious about people and more about ideas."

Marie Curie 


\section{Table of Contents}

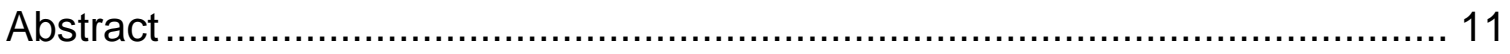

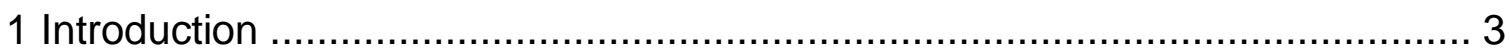

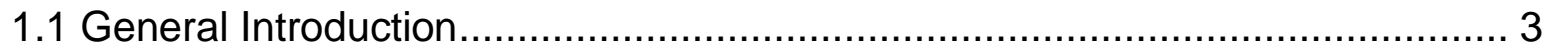

1.1.1 The Brain During Development ………............................................ 3

1.1.2 Oligodendrocytes: The Myelin Engineers in the CNS .............................. 4

1.1.3 The Myelin Basic Protein (MBP) is a Key Component of Compact Myelin . 6

1.1.4 Overlap Between Myelination and Critical Periods .................................. 8

1.2 Influence of Myelination on the Establishment of Behavioural Laterality ........... 9

1.2.1 The Lateralized Brain ...................................................................... 9

1.2.2 Is There a Critical Period for Paw Lateralization? ................................... 11

1.3 Adaptive Myelination and Higher-Brain Functions...................................... 12

1.3.1 Neuronal Activity Triggers Adaptive Myelination .................................... 13

1.3.2 A Peek into the Conundrum that is the Cortical Myelin Heterogeneity ..... 14

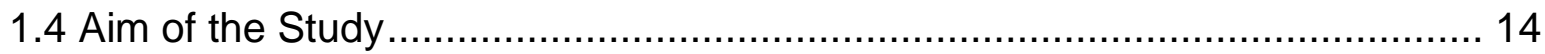

2 Materials and Methods ......................................................................... 17

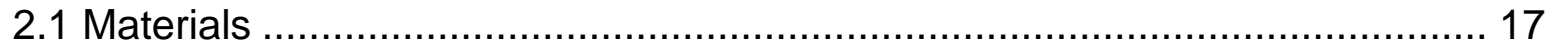

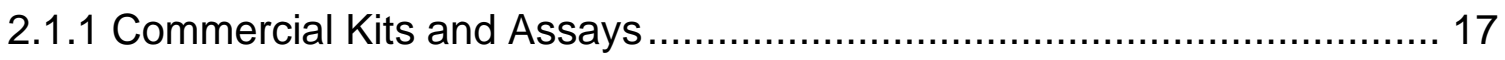

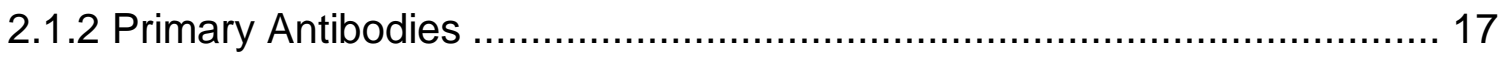

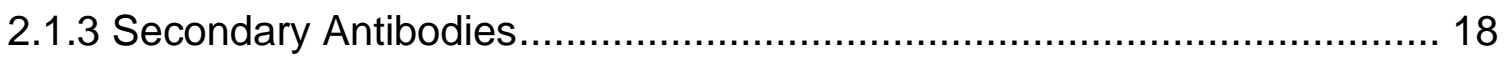

2.1.4 Tissue Collection, SDS PAGE and Immunoblot ................................... 18

2.1.5 Immunohistochemistry and Staining Solutions .................................... 21

2.1.6 Electron Microscopy Buffers and Solutions ........................................ 21

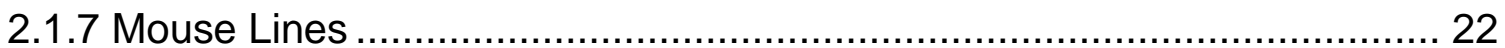

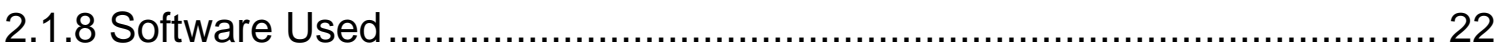

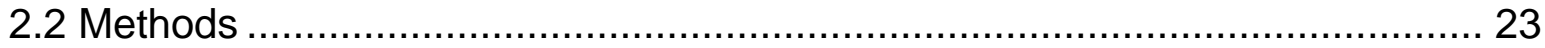

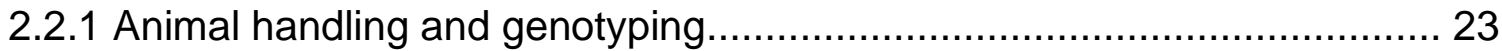

2.2.2 Biochemical Protein Quantification .................................................... 24 
2.2.2.1 Tissue isolation and sample preparation

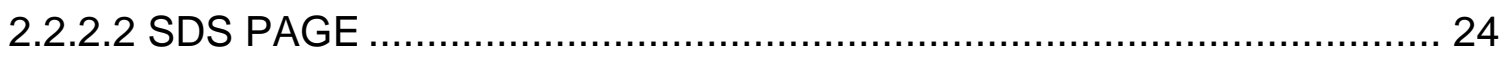

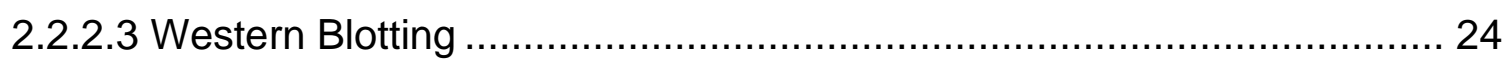

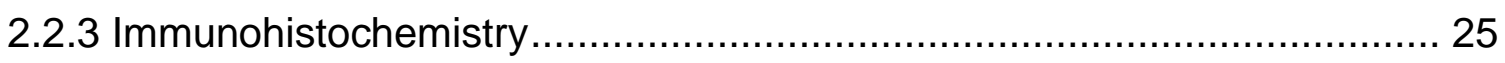

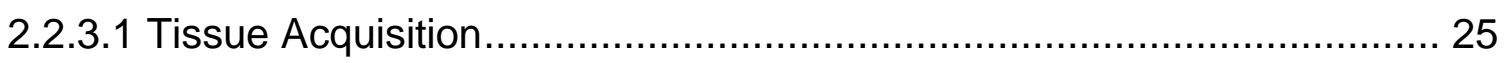

2.2.3.2 Paraffin Embedding of Brains for Immunohistochemistry ..................... 26

2.2.3.3 Immunohistochemistry of Paraffin-Embedded Sections ....................... 26

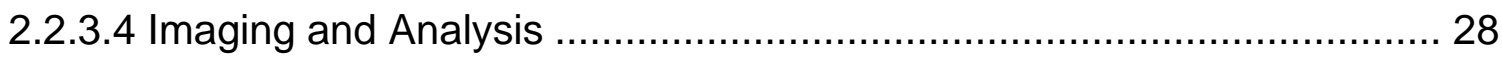

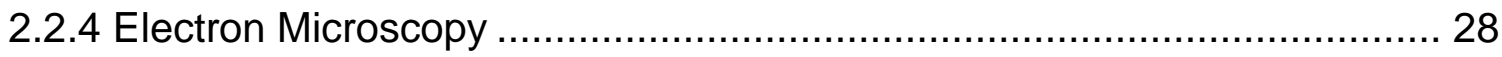

2.2.4.1 Tissue Collection and Epon Embedding ......................................... 28

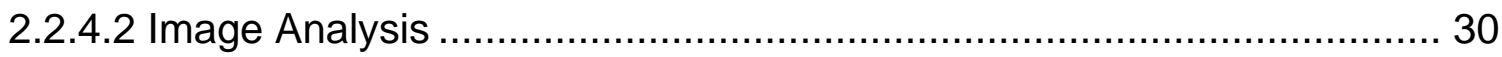

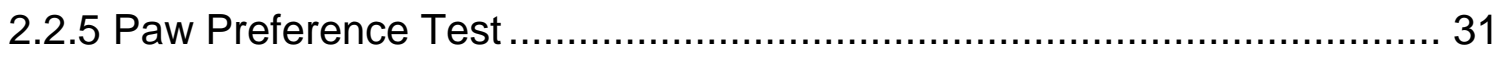

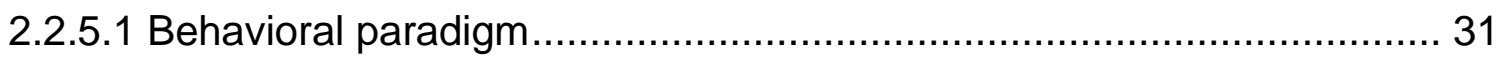

2.2.5.2 Analysing Lateralization in Mice ..................................................... 32

2.2.6 Behavioural Assessment of Higher-Brain Functions............................... 34

2.2.6.1 Mouse Maintenance and Behavioural Tests....................................... 34

2.2.6.2 Cage Behaviour and Health Evaluation ................................................ 36

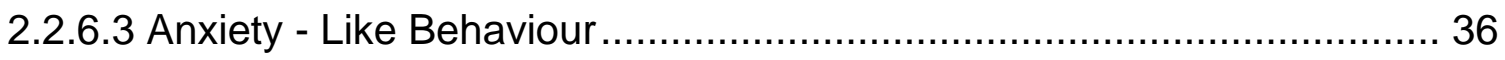

2.2.6.4 Motor Performance and Exploration ................................................... 37

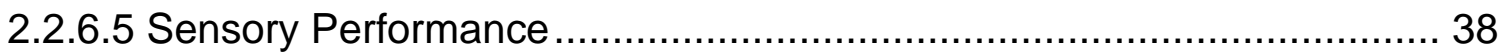

2.2.6.6 Cortical Network Function................................................................. 39

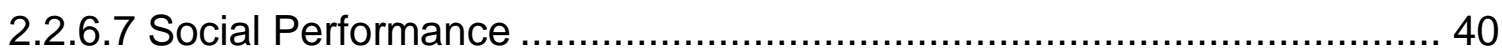

2.2.6.8 Catatonia and Executive Function .................................................... 40

2.2.6.9 Spatial Memory - Morris Water Maze Test............................................ 41

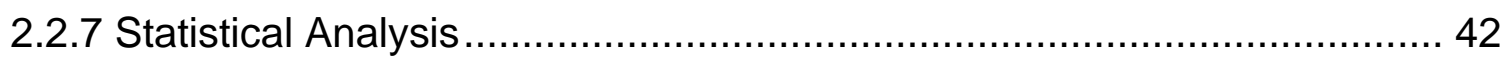

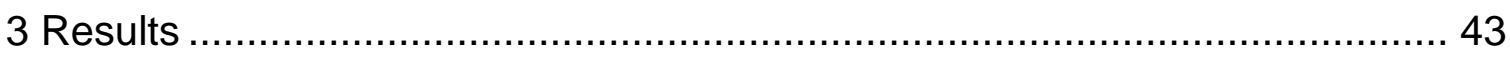

3.1 Young wild-type mice appear to be lateralized by 8 weeks of age ................. 43 
3.2 Young mice lacking compact myelin become lateralized with repetitive testing

3.3 Weekly tested young mice lacking compact myelin show instability with respect to directionality 49

3.4 Shiverer mice that are not tested repetitively during development are still lateralized by 8 weeks of age 51

3.8 Generation of the cortical dysmyelination mouse models 55

3.9 Characterization of the EMXMBP mice 56

3.9.1 EMXMBP mice show a reduction in MBP in the forebrain 56

3.9.2 EMXMBP mice show a mild gliosis phenotype in the corpus callosum .... 59 3.9.3 EMXMBP mice seem to have more OPCs and OLs in the corpus callosum 62

3.9.4 Larger calibre axons are preferentially myelinated compared to smaller calibre axons in the rostral corpus callosum of EMXMBP mice 64

3.10 EMXMBP mice show signs of catatonia at 12 months of age 67

3.11 Chracterization of the FOXMBP mice 69

3.11.1 FOXMBP mice have reduced levels of MBP in the forebrain 69

3.11.2 FOXMBP mice show a mild form of gliosis in the corpus callosum 71

3.11.3 There are more OPCs and OLs in the corpus callosum of FOXMBP mice 74

3.11.4 The rostral corpus callosum of FOXMBP mice is almost entirely dysmyelinated 76

3.11.5 Mice lacking compact myelin the in forebrain show no behavioural lateralization impairment..... 78

4 Discussion and Conclusions 80

4.1 Is there a critical period for the establishment of handedness and does myelin play a role in it? 80

4.2 Characterization of mice lacking compact myelin in the forebrain. 85

4.2.1 EMXMBP and FOXMBP show reduced MBP levels in the cortex and CC 85 
4.2.2 EMXMBP and FOXMBP exhibit mild gliosis and have more oligodendrocytes in regions lacking compact myelin

4.2.3 Ultrastructural analysis of EMXMBP and FOXMBP mice shows regiondependent disparity with regards to dysmyelination ....................................... 89

4.3 What exhibiting sign of catatonia entails for the EMXMBP mice ................... 91

4.4 Does cortical dysmyelination, which extends into the corpus callosum, affect

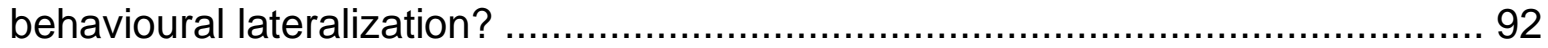

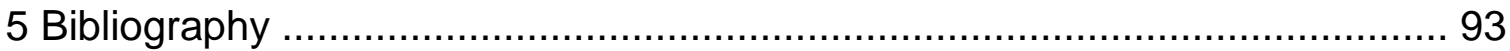

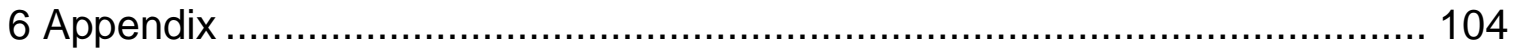

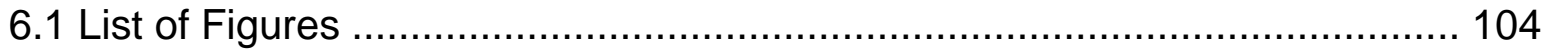

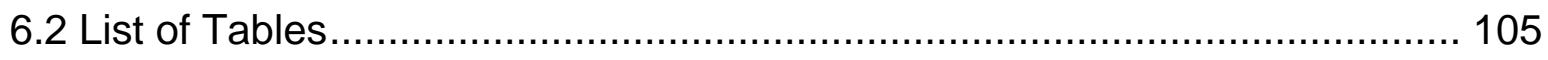

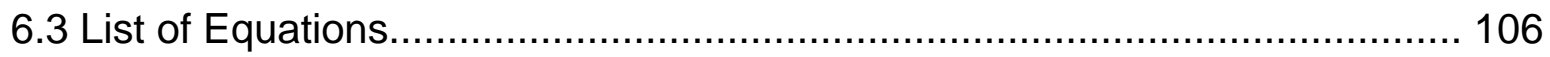

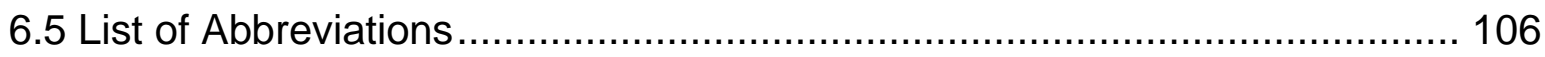

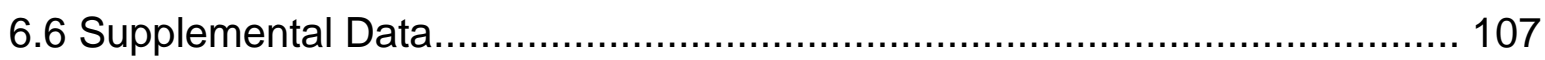

7 Curriculum Vitae .................................................. Error! Bookmark not defined. 


\section{Abstract}

\section{Abstract}

Myelination is a process within the nervous system, where axons are ensheathed by lipid-rich, multi-spiral wraps called myelin. Its main role is to expedite impulse propagation within circuitries and allow for faster saltatory conduction at excitatory regions called the nodes of Ranvier by helping their formation. It begins prior to birth and continues well into adulthood, in humans. At every stage from infancy to adulthood, this process plays a crucial role in both physically and functionally shaping neuronal circuits. Despite being discovered centuries ago, its involvement in forming, fine-tuning and maintaining circuits has only taken centre stage in the recent years. A key question is whether myelinated axons are inherently less plastic, less prone to make new connections with other neurons. To address this question we studied the role of myelin in critical periods. In mice, central nervous system (CNS) myelination peaks between 4 and 8 weeks of age, and overlaps with brief windows of developmental experience-dependent plasticity, called critical periods. The functional role of this overlap is not well understood: is myelin closing the plasticity associated with critical periods? Is it part of the process? Whether this role is permissive or inhibitory is still debatable. To understand the role of myelination in critical periods I chose handedness as a test model. Handedness is established during childhood, when myelination is still progressing, and is dependent on a functional corpus callosum. The corpus callosum is a fibre path that connect both hemispheres and is heavily myelinated in adults. Previous studies in the lab have shown that adult mice lacking myelin do not show paw lateralization. First, to characterize the development of paw lateralization, I tested young wild type mice between 4 and 8 weeks of age using the paw preference behavioural paradigm. I found that wild type mice typically become lateralized by 8 weeks of age. To further assess whether myelination is important for this establishment, I tested young mutant mice lacking compact myelin in the CNS. While these mice are less lateralized than wild type, initially, with practice they become as lateralized as wild type mice. Their handedness, however, in contrast to that of wild type mice, tends to be unstable from test to test during this period. However, this is not observed in adult mice. To address the specific role of the corpus callosum in the establishment of handedness, I generated and tested mice 
lacking compact myelin in the forebrain and, surprisingly, found no evidence of impairment in lateralization.

The forebrain-specific dysmyelinated mice were of particular interest because they can be used to study the role of cortical myelination in higher cognitive functions. In a collaborative effort, colleagues tested these mice for a plethora of behavioural domains such as anxiety, social behaviour, motor deficits, sensorimotor gating, vision, hearing, catatonia and executive function, and found no deficits, except for mild signs of catatonia at older time-points. That too was no longer present at the latest time-point they were tested. Moreover, mice with a complete dysmyelination of the CNS tend to develop seizures that prove fatal at a very young age. Unlike in these mice, forebrain-specific dysmyelination does not affect the lifespan of the mice and hence may provide an interesting insight into adaptations within circuitries and gross morphology of a brain-region surviving without compact myelin. I characterized the general morphology of the mutant mice and found that, interestingly, these mice do not suffer from any major neuropathology or any obvious physiological shortages.

Overall, the data suggests that lack of myelin in the CNS does not prevent apparent development of lateralization in childhood. That the observed lateralization is not stable suggests that this is not 'true' lateralization in the sense we understand it in humans, as a person's trait but rather has a more functional and temporally local characteristic. Myelin, therefore, is crucial in the development of handedness and concomitantly plays a role over the critical period, preventing its closure. Interestingly, unlike mice without any myelin and trained only in adulthood, mice lacking myelin only in the forebrain and part of the corpus callosum had normal lateralization. Moreover, I introduce two new mouse models that lack compact myelin specifically in the forebrain to investigate the role of cortical myelination and its heterogeneity in higher brain functions. 


\section{Introduction}

\subsection{General Introduction}

\subsubsection{The Brain During Development}

The central nervous system (CNS) is comprised of the brain and the spinal cord. The skull generates a microenvironment for the brain by isolating it from the rest of the body. During early embryonic development, neural stem cells (NSCs), located at the ventricular zones (VZ) transition into neuronal progenitor cells (NPCs). The NPCs then differentiate into neurons such as excitatory projection neurons and inhibitory interneurons via a process called neurogenesis. Once neurogenesis is complete, a small subset of the NSCs differentiate into radial glial progenitor cells (RGPs). These further differentiate to form the other cell-types that make up the rest of the brain, the glial cells. This process, which begins prenatally in most mammals, is termed gliogenesis (Beattie and Hippenmeyer, 2017; Rakic, 2009; Stiles and Jernigan, 2010) and continues well into adulthood (Figure 1). Glial cells, consisting of oligodendrocytes (OLs), astrocytes and microglia, make up as much as $50 \%$ of brain cells (von Bartheld et al., 2016). While neurons form connections, called synapses, with one another to transmit and integrate information in the form of action potentials, glial cells help create the microenvironment necessary for formation, survival and maintenance of these circuitries over the course of a being's lifetime.

Astrocytes, the star-shaped glial cells of the brain are the most abundant glial cells and have a plethora of functions ranging from synaptic recycling of neurotransmitters (Allen and Barres, 2009) to buffering the interstitial space in the brain (Walz, 2000). Microglia, unlike OLs and astrocytes are derived from myeloid progenitor cells early in development (Ginhoux and Prinz, 2015). Like macrophages in the peripheral system, microglia possess phagocytic and cytotoxic capabilities and consequently they are the immune cells of the brain. Oligodendrocytes are glial cells that have processes that wrap around axons. These condense into a multi-spiral lipid-rich membrane structure called myelin sheaths (Baumann and Pham-Dinh, 2001). 


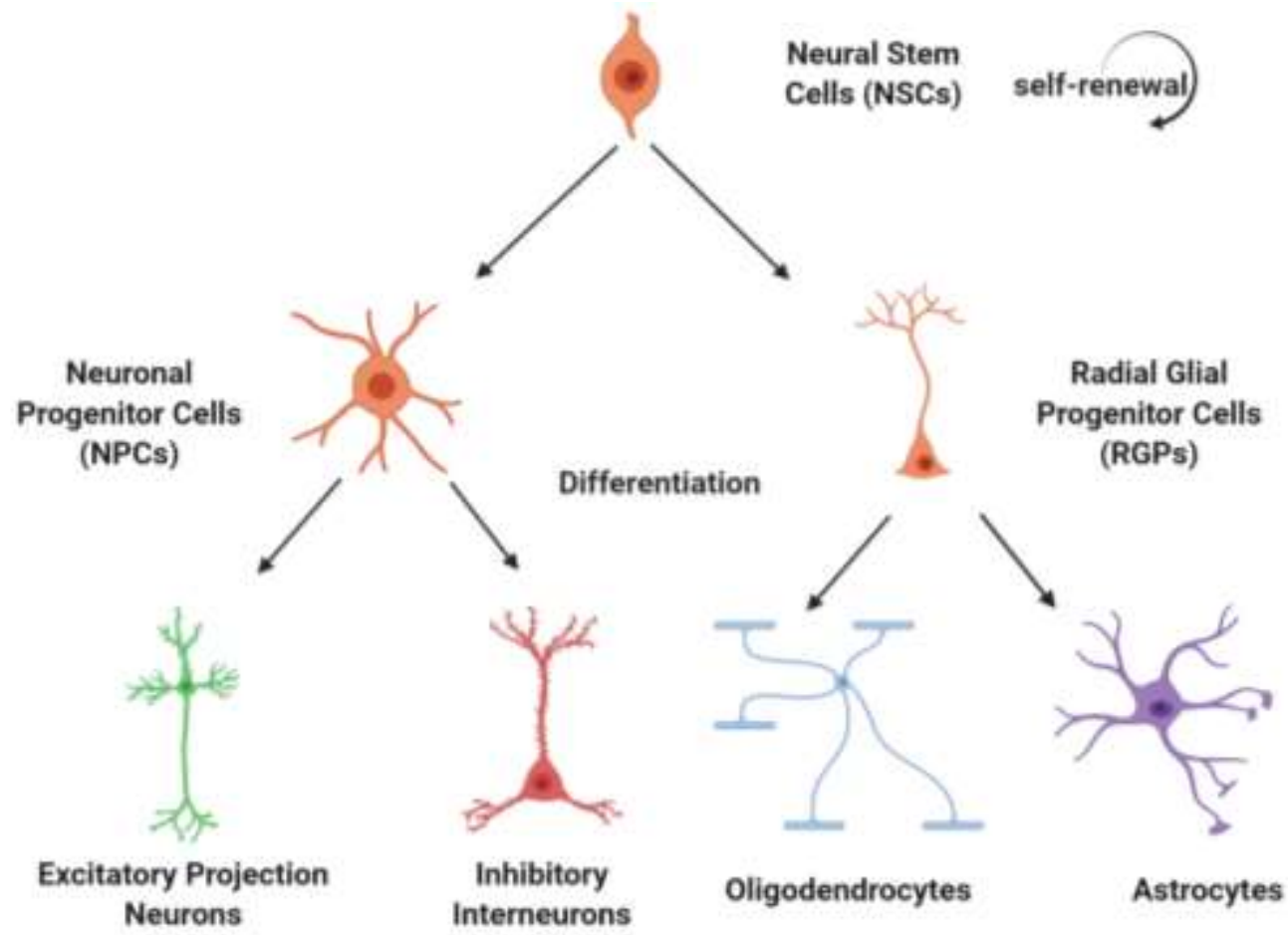

Figure 1: Schematic diagram showing the development of the brain from a single set of stem cells.

The neural stem cells (NSCs) give rise to not only the neuronal populations that comprise the neocortex but also the glial cells that support the formation and maintenance of synapses and circuitries. Created with BioRender.com

\subsubsection{Oligodendrocytes: The Myelin Engineers in the CNS}

In the 1920s, the Spanish neuroscientist Pío del Río Hortega implemented metallic impregnation techniques to describe a group of neuroglia, called oligodendrocytes. OLs are morphologically different from other cell types. These differences include smaller size, dense cytoplasm and nuclei, and lack of intermediate filaments and cytoplasmic glycogen deposits. Moreover, they have a large number of microtubules in their processes which most likely stabilize them during myelination (Baumann and Pham-Dinh, 2001).

The journey of a single oligodendrocyte begins with a precursor called the oligodendrocyte precursor cell (OPC). Since their discovery in 1983 (Raff et al., 1983), our understanding of the mechanisms by which OPCs differentiate to form myelin-forming OLs have come a long way. They arise in the ventricular zones during embryonic development. As the brain further develops, OPCs proliferate and migrate to different regions where they then differentiate into OLs. In particular, 
OPCs retain their ability to proliferate, albeit at a slower pace, in the adult brain, especially in response to aging or injury-induced demyelination. One OL can form up to 60 processes to wrap around multiple segments of different axons to myelinate them (Simons and Nave, 2015) (Figure 2).

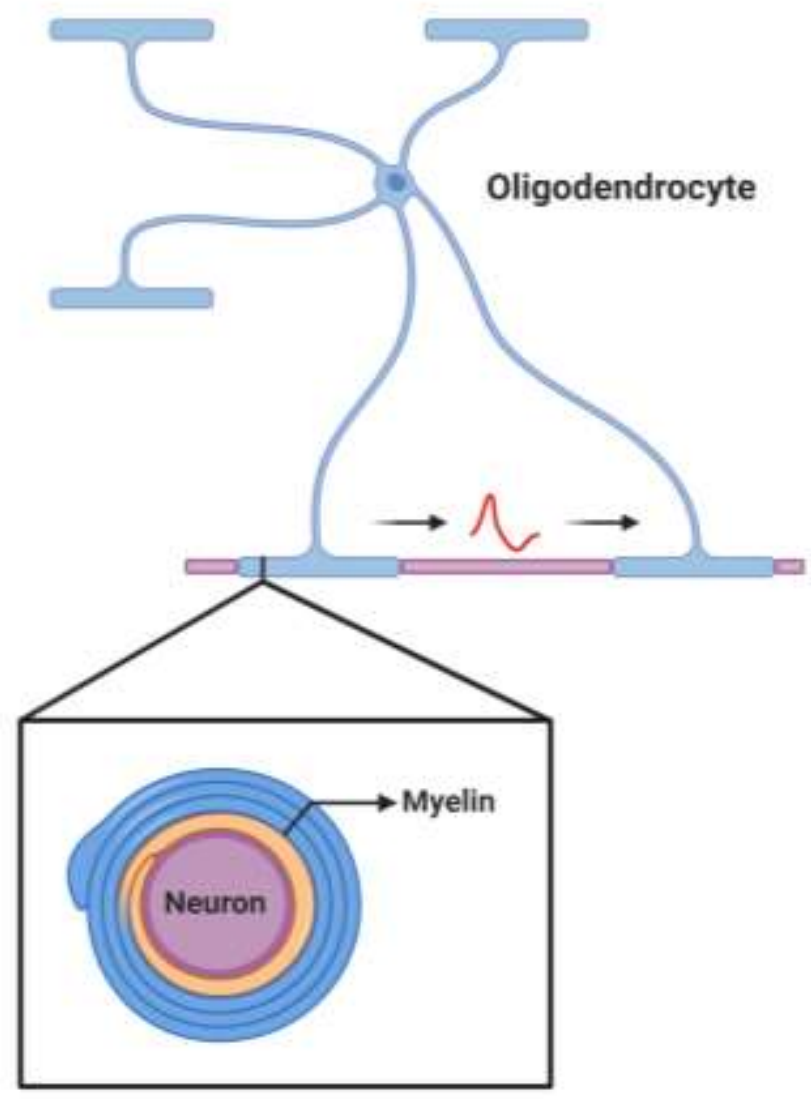

Figure 2: Schematic representation of an oligodendrocyte (OL) myelinating an axonal segment.

The membrane of an oligodendrocyte process wraps around the axon in a multi-spiral manner, extruding the cytoplasm to form the lipid-rich wrappings known as myelin sheath. This is essential especially for long-range axons to expedite the propagation of action potentials and thereby mediate rapid conduction of signals in a circuit. Created with BioRender.com

A subpopulation of OLs, more commonly known as satellite OLs, are present in the grey matter. These OLs, unlike the ones in the white matter (WM), do not myelinate axons. It is postulated that they exist in a quiescent stage, opposed to neurons, regulating the extracellular milieu. In case of a demyelinating event, these perineuronal OLs have the capacity to transition into myelin-forming OLs (Szuchet et al., 2011). 
Once OPCs differentiate into myelin-forming OLs, the transcription of key myelin genes such as Mog, Cnp, Mbp and Mag via the myelin regulatory factor (Myrf) is upregulated. Myrf is a transcription factor that is present in mature, pre-myelinating OLs and is a key regulator of myelin gene transcription. Studies have shown that when Myrf is knocked-out in mice, they are incapable of forming new myelin, reflected in a significant downregulation of important myelin genes (Emery et al., 2009). One such protein that is dependent on Myrf regulated transcription is the myelin basic protein (MBP).

\subsubsection{The Myelin Basic Protein (MBP) is a Key Component of Compact Myelin}

Myelin is a lipid-rich sheath surrounding axons that helps expedite action potential propagation without increasing the diameter. It does so by simultaneously reducing the capacitance and increasing the resistance of the axonal membrane. Myelin, as a compact glial sheath, evolved in vertebrates more than 500 million years ago driven by the evolutionary advantage of faster reflexes for both prey or predator (Nave and Trapp, 2008). MBP is the second most abundant myelin protein in the CNS, constituting nearly $30 \%$ of the myelin protein content by weight. As the OL membrane comes in close contact to itself, in an attempt to form wraps, the two bilayers experience repulsion due to negatively charged phospholipids such as phosphatidylinositol 4, 5 bisphosphate (PIP2).

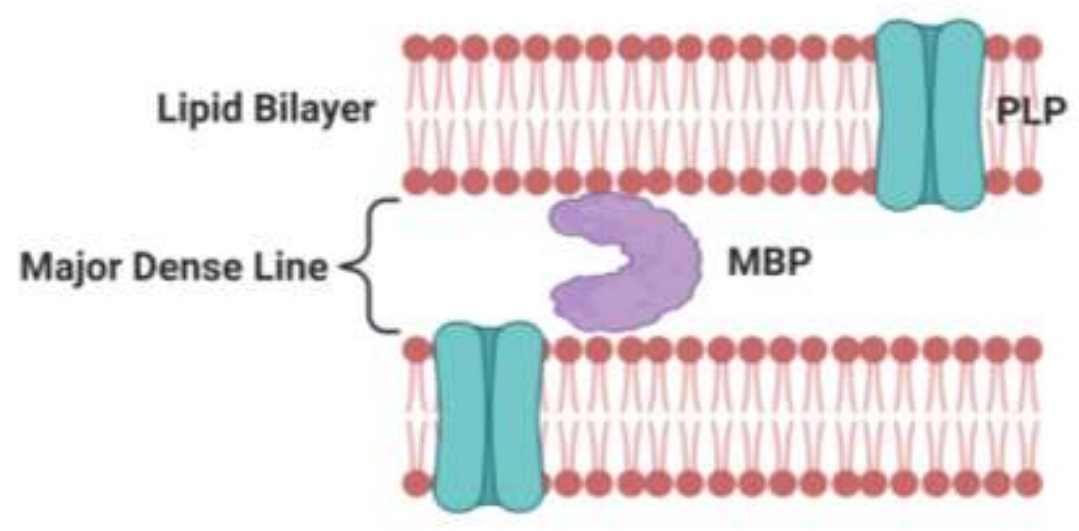

Figure 3: The role of MBP in forming compact myelin.

MBP, as the name indicates, is a highly basic protein that neutralises the negatively charged bilayer membrane of the OL and brings them together to form compact myelin. Created with BioRender.com 


\section{Introduction}

Basic proteins such as MBP help neutralize the charge and bring together the two bilayer membranes in a zippering fashion (Aggarwal et al., 2013; Nave and Werner, 2014). Thus, MBP is best known for its role in forming compact myelin (Figure 3). A member of the 'intrinsically disordered' protein family, it can adapt its structure depending on the binding partner. Due to this ability, it interacts with a myriad of proteins such as actin, Ca2+-calmodulin, clathrin, tubulin and even negatively charged lipids (Boggs, 2006).

MBP is highly conserved across all mammalian species and has at least four isoforms, a consequence of alternative splicing (de Ferra et al., 1985; Harauz and Boggs, 2013).

The shiverer mouse is one of the most researched dysmyelination mouse models in the myelin field. This mouse is a product of a mutation that occurs in the Mbp gene causing a deletion of exons 3 to 7 . The transcribed mRNA is smaller and believed to be more vulnerable to degradation (Molineaux et al., 1986; Roach et al., 1985). The mutation follows an autosomal recessive inheritance pattern. Due to the severe reduction of myelin in both the brain and spinal cord, these mice start showing tremors at two weeks of age and develop tonic seizures, which ultimately result in their early death at roughly three months of age (Chernoff, 1981; Kirschner and Ganser, 1980). During their short lifespan, these mice show a plethora of morphological and physiological abnormalities such as loosely packed myelin, hypomyelinated, and even unmyelinated axons in typically heavily myelinated regions of the CNS. This is in stark contrast to their peripheral nervous system (PNS) which appears to be normally myelinated (Rosenbluth, 1980), owing to the fact that P0, rather than MBP, is the key component of PNS compact myelin (Harauz and Boggs, 2013). In addition, ion channel clusters at the paranodes and nodes of Ranvier are more diffused due to the loosely packed myelin (Rasband and Trimmer, 2001; Sinha et al., 2006).

Since this mouse model is so central to the study of myelination and the neurodegenerative diseases associated with its degradation, it became a key model, which I used to address the questions raised in this study. 


\subsubsection{Overlap Between Myelination and Critical Periods}

Myelination begins prenatally in most vertebrates and continues well into adulthood. A period of extraordinary plasticity lasting days in mice (Katz, 1999; Lo et al., 2017; Nakamura et al., 2020) and weeks to years in humans (Sharma et al., 2002), precedes myelination. During this period, the neural circuits form at an unprecedented rate. Around this time, external stimuli play a crucial role in shaping, consolidating and even pruning circuitries. The brief windows of experiencedependent plasticity are called critical periods. However, not all circuits formed at this time are maintained throughout an individual's life. Unlike processes such as neurogenesis, which slows down significantly in adulthood, myelination is more easily stimulated by neuronal activity irrespective of age.

First described in 1970 by two neurophysiologists who performed elegant experiments on kittens to show ocular dominance plasticity (ODP), Torsten Wiesel and David Hubel postulated that this kind of visual plasticity was restricted to a brief window during development and that it is driven by external stimuli (Hubel and Wiesel, 1970). Since then, critical periods have been described for several skills such as language learning and sensory processes such as the auditory and vestibular systems.

However, this form of unbridled plasticity can be detrimental to the system under some circumstances. Circuits need consolidation to work reliably well into

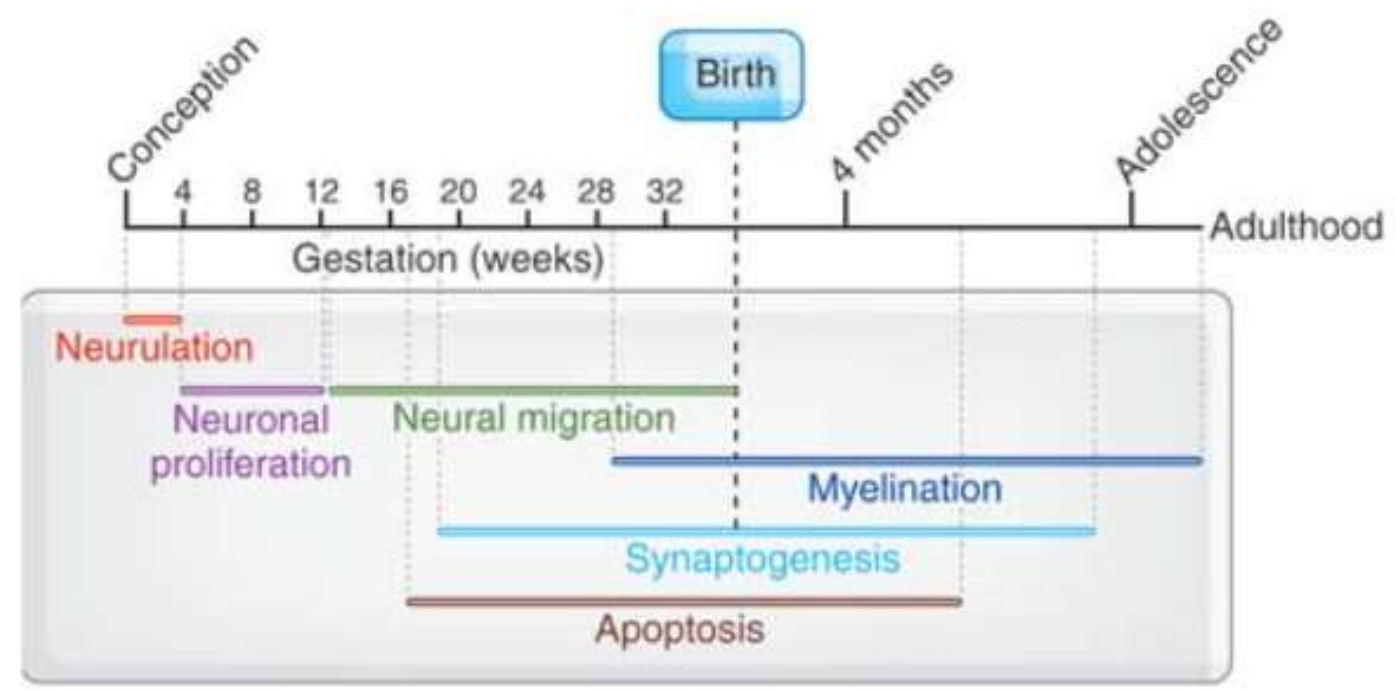

Figure 4: A timeline of different brain development events in a human.

Starting from its conception, this schematic follows the different stages of brain development in a human. 8 weeks after conception, the first neural progenitors are seen which then go on to proliferate and differentiate into the neurons and glial cells that make up the brain at birth and into adulthood. Image taken from Tau and Peterson, 2010. 
adulthood. As myelination follows a period of synaptic plasticity (Tau and Peterson, 2010), where several synapses are pruned while others are maintained, it is fair to assume that myelin may play a role in regulating critical periods (Figure 4). Some studies have shown that certain myelin proteins may play a role in critical period closure. They showed that mice lacking the Nogo-66 receptor $(\mathrm{NgR})$ show extended adult plasticity in their visual cortex in response to monocular deprivation. Thus, key myelin proteins that function as circuit consolidators during plasticity, may signal the closure of critical periods (Holmes et al., 2012; McGee et al., 2005; Stephany et al., 2016; Yiu and He, 2006).

\subsection{Influence of Myelination on the Establishment of Behavioural Laterality}

Lateralization of certain skills is a consequence of structural or functional asymmetries between the two hemispheres of the brain and are often attributed to lateralization in cortical regions (Güntürkün and Ocklenburg, 2017). The corpus callosum (CC), the most heavily myelinated tract in the brain (Gazzaniga, 2000), is believed to play in role establishing and maintaining these asymmetries (Cassells et al., 1990; van der Knaap and van der Ham, 2011). The study of brain asymmetry began in 1865 when Pierre Paul Broca, a French physician observed that patients with left hemisphere lesions in a particular region (later known as Broca's area) experienced speech impairment. This was not seen in patients with a lesion in their right hemisphere. He made the claim, "Nous parlon avec l'hemisphère gauche" ("We speak with the left hemisphere"; (Broca, 1865)).

\subsubsection{The Lateralized Brain}

How a vertebrate body develops into an asymmetric system has long been a mystery to those who study development. Only in recent years, some answers to how this is genetically mediated have emerged. Hemispheric asymmetries on the structural level are observed by expression of certain genes in a region-specific left-right manner (Karlebach and Francks, 2015). At the macrostructural level, certain brain regions have been reported to be asymmetrically bigger or smaller in size and shape (Amunts, 2010; Güntürkün and Ocklenburg, 2017). 
Functional asymmetries arise most predominantly in the language centre of the brain (Corballis, 2012). However, other processes are also affected such as auditory processing (Tervaniemi and Hugdahl, 2003), spatial-visual processing (Vogel et al., 2003), motoric functions (Goodale, 1988), attention (Brooks et al., 2014), preference of hand for writing (Papadatou-Pastou et al., 2008), foot preference to kick a ball with (Carey et al., 2001), emotional processing (Grimshaw and Carmel, 2014), and even memory (Cabeza, 2002). In fact, people also show a preference in the direction to which they tilt their heads while kissing (Güntürkün, 2003). Since these asymmetries exist in such a multifaceted manner, understanding and thereby correlating differences on the behavioural level to the functional and structural level is not easy.

The CC is phylogenetically speaking a relatively new projection seen explicitly in eutherians (placental mammals). Noneutherian animals such as monotremes and marsupials lack a CC (Figure 5). Their interhemispheric connections are maintained by the anterior commissure. Recent studies using diffusion tensor magnetic resonance imaging (MRI) have shown that despite having an additional tract that connects the two hemispheres, eutherians share characteristics of interhemispheric communications with noneutherians. This suggests that the evolution of the CC in mammals was independent of the connectome between hemispheres and that this dates approximately 80 million years before the evolution of the CC (Suárez et al., 2018).

One of the most studied and interesting forms of lateralization is that of handedness. In humans, $90 \%$ of the population shows a right-hand preference. This is been seemingly consistent for nearly 5000 years (Coren and Porac, 1977). Foetuses show a preference in thumb-sucking, in gestation week 15 , which is consistent with the dominant hand for fine motoric tasks after birth (Hepper et al., 1991, 2005). However, other species of animals have also shown preference of one limb over the other with respect to certain tasks (Scharoun and Bryden, 2014). When does this limb laterality develop? How early is it visible in other animals? 


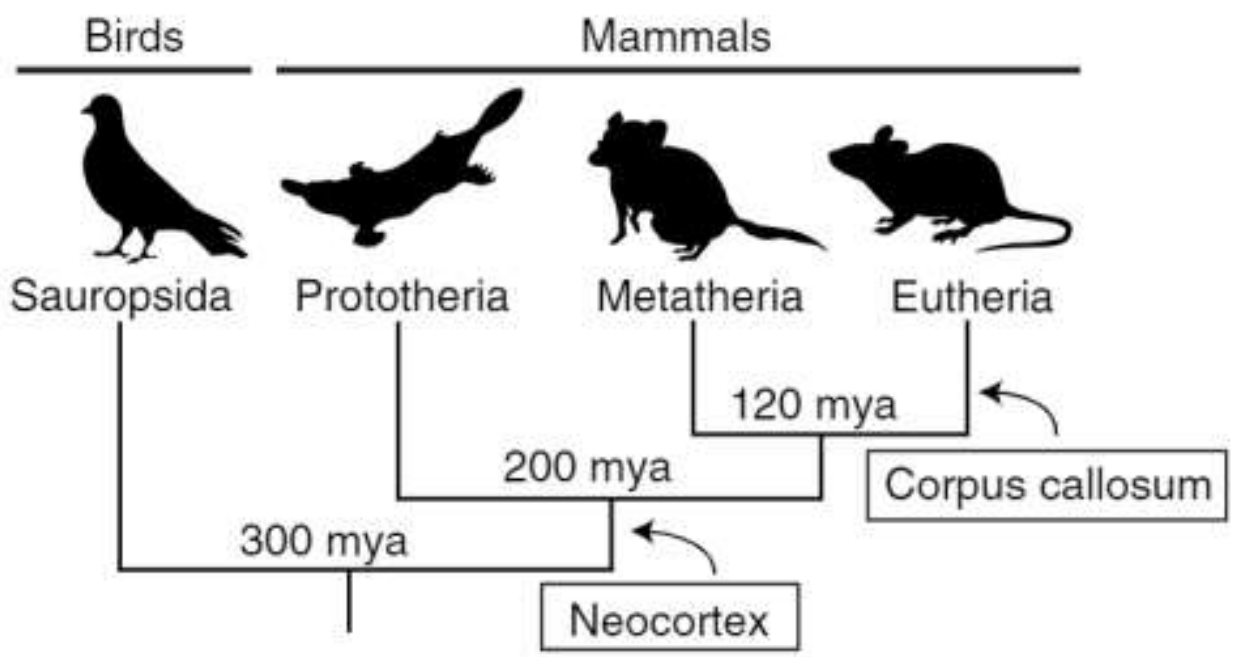

Figure 5: Evolution of interhemispheric connections.

Schematic showing the phylogenetic relationship between noneutherians (monotremes and marsupials) and eutherians (placental mammals) with respect to the evolution of the neo cortex and the corpus callosum. Image taken from Suárez et al., 2018.

\subsubsection{Is There a Critical Period for Paw Lateralization?}

In the 1970s, Robert L. Collins developed an elegant technique to observe paw lateralization in inbred strains of laboratory mice (Collins, 1968, 1969a, 1969b, 1975). He put the mice in small boxes with a small tube on one of the walls. Through this tube, he provided mice with food that they had to retrieve by extending their paws. Only one paw could be inserted at a time and, hence, he could assess which paw the mice preferred to retrieve their reward. After testing hundreds of mice from many inbred strains commonly used in laboratories across the world, he discovered that depending on the strain, mice tended to show a certain population distribution with regards to the directionality as well as the full extent to which they were lateralized.

Later, when a strain of mice with a congenital absence of the $\mathrm{CC}$ were shown to have a reduced paw lateralization compared to control mice from other strains, it indicated that interhemispheric transfer of information mediated by the CC plays a substantial role in paw lateralization (Gruber et al., 1991).

If the CC does indeed play a role in dictating paw lateralization and since it is heavily myelinated, what role does myelin play in the establishment of laterality? In addition, is this role dependent on a time-specific window of development? Thus, from here on I will address paw lateralization as behavioural laterality, expanded further in the 


\section{Introduction}

3 Results section.

\subsection{Adaptive Myelination and Higher-Brain Functions}

While developmental myelination plays an essential role in pruning and consolidating newly formed circuitries, adaptive myelination is more of consequence in the adult brain. Many studies in the recent years have shown that skill acquisition is reinforced by formation of new myelin. In humans, MRI studies show structural changes in WM of people learning new skills such as learning to play an instrument or juggling (Bengtsson et al., 2005; Bergles and Richardson, 2015; Scholz et al., 2009). In mice, it was shown that ablating the ability to form new myelin in adulthood resulted in impairment of a complex motor learning task (McKenzie et al., 2014). Since approximately $40 \%$ of newly formed OLs survive long term in the adult WM (Young et al., 2013), it is safe to assume that they may play a role in adaptive myelination. However, it is yet to be understood whether these OLs participate in de novo myelination or act by changing the length and number of internodes. Recent findings suggest that differentiating OLs generate internodes in a rather small time window without further altering these internodes (Czopka et al., 2013; Young et al., 2013).

Higher brain functions consist of two components. First, cognition which encompasses speech and language, memory, perception and attention, judgement, planning and decision-making. The second aspect is behaviour, which is essentially the manifestation of the cognitive functions in combination with personality. Higher brain functions mediated by the forebrain are complex and 


\section{Introduction}

involve the interaction between the different regions of the cortex both within and between the two hemispheres. Whether and how these are governed by myelination are two pivotal questions that still need to be answered.

\subsubsection{Neuronal Activity Triggers Adaptive Myelination}

Since it is known that myelination regulates neuronal activity, it is likely that the converse also holds true. In the 1960s, Gyllensten and Malmfors demonstrated that the optic nerves of mice reared in the dark were less myelinated. They were the first to suggest the role of neuronal activity in driving myelination (Choi et al., 2019; Gyllensten and Malmfors, 1963). Later studies also showed that increasing neuronal firing rate led to an increase in myelin thickness whereas blocking neuronal firing reduced it (Demerens et al., 1996). More recently, it was shown that optogenetic stimulation of neurons results in remodelling of myelin microstructures and OL dynamics (Gibson et al., 2014). Furthermore, environmental components such as social isolation, enriched housing and motor learning also play a role in promoting adaptive myelination (Hughes et al., 2018; Makinodan et al., 2012; McKenzie et al., 2014; Xiao et al., 2016; Zhao et al., 2012). While these studies provide ample evidence, the mechanisms with which neuronal activity promotes myelination are yet to be elucidated.

The need for activity-dependent myelination arises from many different hypotheses. The possibility of continued neuroplasticity, in adulthood, mediated by myelination in an activity-dependent manner is not just exciting but relevant in case of demyelinating diseases such as MS (Almeida and Lyons, 2017; Purger et al., 2016). In addition, OLs have been shown to provide metabolic support to axons. OLs undergo glycolysis to produce lactate which is then shuttled into axons to be locally utilized for ATP production (Fünfschilling et al., 2012). This reduces the distance metabolites need to travel from the neuronal cell body to the axons. Neuronal activity was also shown to stimulate uptake of glucose by OLs to further expedite ATP production in axons (Saab et al., 2016).

Considering these findings, one may conclude that adaptive myelination is a dynamic yet tightly regulated and elegant process to rewire some circuits and consolidate others. How do higher-brain functions change in the absence of 
compact myelin formation and is myelination during development important in driving or maintaining these behaviours during adulthood?

\subsubsection{A Peek into the Conundrum that is the Cortical Myelin Heterogeneity}

Thickness of myelin is not the only thing that determines the conduction velocity, but also the length of the axon it wraps. The number of internodes, which are the myelin-wrapped segments of the axon, are determined within a very narrow time window during development and rarely change drastically over time (Czopka et al., 2013). However, a reduction in internode length with an increase in the number of internodes is seen in the aging brain (Lasiene et al., 2009; Peters and Kemper, 2012). The neocortex has been reported to show a staggering amount of heterogeneity with respect to myelination. Both excitatory pyramidal neurons and inhibitory interneurons have internodes followed by long stretches of unmyelinated, long-range axons (Micheva et al., 2016; Tomassy et al., 2014). How this heterogeneity develops and whether it persists over time are open questions. A single pyramidal neuron spanning the different layers of the cortex exhibits this heterogeneity depending on the cortical layer. For example, the superficial layers (Layer II/III) tend to have more unmyelinated segments compared to the deeper layers (Tomassy et al., 2014).

This is particularly interesting when one considers the complex behaviours and sensory and motor functions one exhibits on a regular basis. These behaviours are not just controlled by of one specific region. They involve complex circuitries between different regions of the brain, both cortical and subcortical. The circuits need to be well tuned for reliable and consistent output. What are the mechanism underpinning this? What role does myelination play? Using genetic manipulation techniques, I generated a mouse line that lacks compact myelin in a forebrainspecific manner to help answer this question.

\subsection{Aim of the Study}

Once the foundation of the brain is formed during embryonic development, a range of dynamic processes commence after birth to form the complex networks we call neuronal circuits. However, these circuits are not formed perfectly. They are amenable to changes such as synaptic pruning, consolidation, and rewiring. 
Exogenous environmental stimuli such as light, sound, odours and even social interactions also contribute to the above-mentioned dynamic processes. They are of paramount consequence during brief windows of plasticity called critical periods. Since, in mice, many of these critical periods coincide with the peak of myelination I set out to investigate the role it may play in shaping the duration of critical periods. For this study, I chose to focus initially on behavioural lateralization of paw usage in mice. Using a modified version of the behavioural paradigm introduced in the late 1960s (Collins, 1968, 1969b, 1975) to assess paw preference in young mice. I found that there is a period during which behavioural lateralization is progressively established in wild-type mice. To address the role myelin may play in this establishment, I turned to the shiverer mouse model. One caveat of this model is that these mice show a dysmyelination phenotype in the entire CNS, which results in a short lifespan (Chernoff, 1981). Moreover, it is difficult to say reliably whether the effects we see are due to the lack of compact myelin in the CC and the cortex. Using the cre-loxP system of genetic manipulation (Sauer, 1987; Sauer and Henderson, 1988), I was able to generate two mouse lines that lack compact myelin specifically in the forebrain. This means these mice do not experience tremors and seizures that allow them to age as long as wild-type mice do. This also helped to ensure that the behavioural changes we hypothesized to see would be expressly due to cortical myelination deficits and not due to structures anywhere else in the CNS. Since shiverer mice tend to have such a short lifespan, it is not known what the structural and morphological consequences, of such a prolonged form of dysmyelination, are. Using techniques such as immunohistochemistry and biochemical protein estimation, I confirmed just how much of the protein MBP was knocked out in the forebrain. I investigated the ultrastructural changes in the $\mathrm{CC}$ of these mice with electron microscopy. Moreover, in collaboration with the behavioural team of Prof. Dr. Dr. Hannelore Ehrenreich, I analysed different facets of behavioural deficits such as anxiety, catatonia and executive dysfunction, sensorimotor impairment, etc. By implementing these techniques, I was able to get a general picture of the health and disease status of the mouse lines and characterize them. 
Introduction 


\section{Materials and Methods}

\subsection{Materials}

\subsubsection{Commercial Kits and Assays}

Table 1: List of commercial assays and kits used

\begin{tabular}{|c|c|c|}
\hline Kit & Usage & Manufacturer \\
\hline $\begin{array}{c}\text { DC Protein Assay } \\
\text { (Lowry) }\end{array}$ & $\begin{array}{c}\text { Protein quantification for } \\
\text { Western Blots }\end{array}$ & Bio-Rad \\
\hline nexttec $^{T M}$ & DNA isolation & 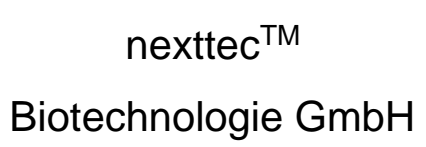 \\
\hline Universal LSAB2 Kit & DAB IHC & Dako \\
\hline $\begin{array}{c}\text { VECTASTAIN ELITE® } \\
\text { ABC Kits }\end{array}$ & DAB IHC & Vector Laboratories \\
\hline DAB Zytomed Kit & DAB IHC & $\begin{array}{c}\text { Zytomed Systems } \\
\text { GMBH }\end{array}$ \\
\hline
\end{tabular}

\subsubsection{Primary Antibodies}

Table 2: List of primary antibodies used for immunohistochemistry and western blots.

\begin{tabular}{cccc} 
Antibody & Species & Use & Manufacturer \\
\hline App & $\begin{array}{c}\text { Monoclonal } \\
\text { mouse }\end{array}$ & $1: 1000$ & Millipore \\
CD3 & Monoclonal rat & $1: 250$ & Abcam \\
GFAP & $\begin{array}{c}\text { Monoclonal } \\
\text { mouse }\end{array}$ & $1: 200$ & Novocastra \\
Iba1 & Polyclonal rabbit & $1: 750$ & Wako \\
Mac3 & Monoclonal rat & $1: 400$ & BD Pharmingen \\
& Polyclonal rabbit & $1: 8000(\mathrm{IHC})$ & Custom made \\
MBP & Polyclonal rabbit & $1: 100$ & (Kathrin Kusch) \\
Olig2 & John Alberta
\end{tabular}




\section{Results}

$\begin{array}{cccc}\text { CAll } & \text { Monoclonal rat } & 1: 1000 & \text { R\&D Biosystems } \\ \text { CNPase } & \begin{array}{c}\text { Monoclonal } \\ \text { mouse }\end{array} & 1: 5000(\mathrm{WB}) & \text { Sigma }\end{array}$

\subsubsection{Secondary Antibodies}

Table 3: List of secondary antibodies used for immunohistochemistry and western blots.

\begin{tabular}{|c|c|c|c|}
\hline Antibody & Species & Use & Manufacturer \\
\hline $\begin{array}{c}\text { a-rabbit Alexa } \\
555\end{array}$ & Donkey & $1: 1000$ & Dianova \\
\hline $\begin{array}{c}\text { a-mouse Alexa } \\
633\end{array}$ & Donkey & $1: 1000$ & Dianova \\
\hline $\begin{array}{c}\text { Biotinylated Anti- } \\
\text { Rat IgG }(\mathrm{H}+\mathrm{L})\end{array}$ & Goat & $1: 100$ & $\begin{array}{c}\text { Vector } \\
\text { Laboratories }\end{array}$ \\
\hline $\begin{array}{l}\text { a-mouse IgG H\&L } \\
\text { (IRDye® 680RD) }\end{array}$ & Goat & $1: 1000$ & abcam \\
\hline $\begin{array}{l}\alpha \text {-mouse IgG H\&L } \\
(\text { IRDye® } 800 \mathrm{CW})\end{array}$ & Goat & $1: 1000$ & abcam \\
\hline
\end{tabular}

\subsubsection{Tissue Collection, SDS PAGE and Immunoblot}

Table 4: Buffers and solutions used for tissue collection.

\begin{tabular}{ccc} 
Name & Content & Concentration \\
\hline \multirow{2}{*}{ 10X Phosphate-buffered } & $\mathrm{NaCl}$ & $1.7 \mathrm{M}$ \\
saline (PBS) & $\mathrm{KCl}$ & $34 \mathrm{mM}$ \\
& $\mathrm{Na}_{2} \mathrm{HPO}_{4} \times 2 \mathrm{H}_{2} \mathrm{O}$ & $40 \mathrm{mM}$ \\
& $\mathrm{K}_{2} \mathrm{HPO} 4$ & $18 \mathrm{mM}$ \\
& & $\mathrm{pH} 7.2$
\end{tabular}


Table 5: Buffers and solutions used for SDS PAGE

\begin{tabular}{|c|c|c|}
\hline Name & Content & Concentration \\
\hline \multirow{6}{*}{ Modified RIPA buffer } & Tris/HCl, $\mathrm{pH} 7.5$ & $50 \mathrm{mM}$ \\
\hline & $\mathrm{NaCl}$ & $0.15 \mathrm{M}$ \\
\hline & EDTA & $1 \mathrm{mM}$ \\
\hline & Sodium deoxycholate & $0.5 \%(w / v)$ \\
\hline & Triton X-100 & $1 \%(v / v)$ \\
\hline & Protease inhibitor & 1 tablet $/ 10 \mathrm{~mL}$ \\
\hline \multirow{5}{*}{$\begin{array}{l}\text { SDS separating gel } \\
\qquad(15 \%)\end{array}$} & Acrylamide/Bisacrylamide & $15 \%(v / v)$ \\
\hline & $29: 1$ & $0.4 \mathrm{M}$ \\
\hline & Tris/HCl pH 8.8 & $0.1 \%(w / v)$ \\
\hline & SDS & $0.03 \%(w / v)$ \\
\hline & $\begin{array}{l}\text { APS } \\
\text { TEMED }\end{array}$ & $0.08 \%(v / v)$ \\
\hline \multirow{6}{*}{ SDS stacking gel (5\%) } & Acrylamide/Bisacrylamide & $5 \%(v / v)$ \\
\hline & $29: 1$ & $125 \mathrm{mM}$ \\
\hline & Tris/HCl pH 6.8 & $0.1 \%(w / v)$ \\
\hline & SDS & $0.05 \%(w / v)$ \\
\hline & APS & $0.1 \%$ \\
\hline & TEMED & \\
\hline \multirow{4}{*}{ 4x SDS sample buffer } & Glycerol & $40 \%(v / v)$ \\
\hline & Tris/HCl pH 6.8 & $240 \mathrm{mM}$ \\
\hline & SDS & $8 \%(w / v)$ \\
\hline & Bromophenol blue & $0.04 \%(w / v)$ \\
\hline
\end{tabular}




\section{Results}

10x SDS running buffer

(Laemmli buffer)
Tris base

Glycine

SDS
$250 \mathrm{mM}$

$1.9 \mathrm{M}$

$1 \%(w / v)$

Table 6: List of buffers and solutions used for Immunoblot

\begin{tabular}{|c|c|c|}
\hline Name & Content & Concentration \\
\hline \multirow{3}{*}{$1 x$ transfer buffer } & Tris base & $25 \mathrm{mM}$ \\
\hline & Glycine & $190 \mathrm{mM}$ \\
\hline & Methanol & $20 \%(v / v)$ \\
\hline \multirow{3}{*}{$\begin{array}{c}\text { 1x TBST (TBS-Tween } \\
20)\end{array}$} & Tris pH 7.5 & $20 \mathrm{mM}$ \\
\hline & $\mathrm{NaCl}$ & $150 \mathrm{mM}$ \\
\hline & Tween 20 & $0.1 \%(\mathrm{v} / \mathrm{v})$ \\
\hline \multirow{3}{*}{$\begin{array}{c}\text { Fast Green washing } \\
\text { solution }\end{array}$} & Methanol & $30 \%(v / v)$ \\
\hline & Acetic Acid & $6.7 \%(\mathrm{v} / \mathrm{v})$ \\
\hline & $\mathrm{ddH} 20$ & Made up to $1 \mathrm{~L}$ \\
\hline \multicolumn{3}{|c|}{ Fast Green stock } \\
\hline $0.1 \%$ Fast Green & solution & $0.1 \%(\mathrm{v} / \mathrm{v})$ \\
\hline (working solution) & $\begin{array}{c}\text { Fast Green washing } \\
\text { solution }\end{array}$ & Made up to $50 \mathrm{~mL}$ \\
\hline \multirow{2}{*}{$\begin{array}{l}\text { Immunoblot blocking } \\
\text { solution }\end{array}$} & \multirow{2}{*}{ Non-fat dry milk powder } & $5 \%(w / v)$ \\
\hline & & In TBST \\
\hline Product & & Manufacturer \\
\hline
\end{tabular}

Complete Mini protease inhibitor

PageRulerTM Plus Prestained Protein Ladder
Roche Diagnostics

GE Healthcare Life Science 
PVDF Membrane Imobilon ${ }^{\circledR}$-FL $\mathrm{P} 0.45 \mu \mathrm{m}$

Merck Millipore Ltd.

\subsubsection{Immunohistochemistry and Staining Solutions}

\section{Paraformaldehyde}

$4 \%$ (w/v) Paraformaldehyde (PFA)

$0.1 \mathrm{M}$ Phosphate buffer

Citrate buffer $(0.01 \mathrm{M} \mathrm{pH} \mathrm{6.0;} \mathrm{for} \mathrm{antigen} \mathrm{retrieval)}$

$1.8 \mathrm{mM}$ Citric acid

8.2 mM Sodium citrate

Always prepared fresh

Blocking solution (paraffin sections)

$10 \%(v / v)$ goat serum

$1 \times$ PBS

\section{Mounting Media}

Eukitt Kindler (Kindler, Freiburg, Germany) for DAB labelled paraffin sections Aqua-Poly/Mount Polysciences (Polyscienes, Eppelheim, Germany) for fluorescent-labelled sections

\subsubsection{Electron Microscopy Buffers and Solutions} Karlsson-Schultz Fixative

$4 \%(w / v)$ PFA

$2.5 \%(v / v)$ Glutaraldehyde

Epoxy Resin (Epon) sample embedding

Glycidether $100-171.3 \mathrm{~g}$ 
Dodecenyl succinic anhydride (DDSA) - $115 \mathrm{~g}$

Methyl nadic anhydride (MNA) - $89 \mathrm{~g}$

Mix using magnetic stirrer for 10 minutes

DMP-30 $6.5 \mathrm{~mL}$

Mixed using magnetic stirrer for 20 minutes

\section{Richardson's Methylene Blue/ Azure II Blue}

Richardson's methylene blue/Azure II blue stains for lipid-rich regions in electron microscopy semi-thin sections. Stock solutions consist of:

$1 \%(w / v)$ Azure II (Merck, Darmnstadt, Germany) in dd $\mathrm{H}_{2} \mathrm{O}$ (stock solution I).

$1 \%(w / v)$ Methylene blue (Merck, Darmnstadt, Germany) in 1\% sodium borate (stock solution II).

Working solution is prepared using a 1:1 mixture with a syringe and filtered directly before use. It is usable for up to 24 hours.

\subsubsection{Mouse Lines}

The following mutant mouse models were used for this study:

Wild-type C57BL/6J mice - all mouse lines were bred on this background.

Shiverer mice - mice lacking both alleles of MBP.

$E m \times 1 \mathrm{Cre} / \mathrm{Mbp}^{f / f l}$ - conditional knockout of MBP in a forebrain-specific manner by crossing Emx1-IRES-Cre (Gorski et al., 2002) and Mbp flox (Meschkat et al., 2020) mice.

FoxG1Cre/Mbp ${ }^{f / f l}$ - conditional knockout of MBP in a forebrain-specific manner by crossing FoxG1-IRES-Cre (Kawaguchi et al., 2016) and Mbp flox mice.

\subsubsection{Software Used}

\begin{tabular}{cc} 
Software & Manufacturer \\
\hline Adobe Illustrator CS5 & Adobe \\
GraphPad Prism 8 & www.graphpad.com \\
ImageJ & $\mathrm{NIH}$
\end{tabular}


ZEN 3.2 Lite

Excel 2016

Matlab 2017

ZEN 2012

bioRENDER

R studio v1.2.5019
Zeiss

Microsoft

Mathworks

Zeiss

bioRENDER

RStudio Inc.

\subsection{Methods}

\subsubsection{Animal handling and genotyping}

All experimental animals were bred and housed in the animal facility of the Max Planck Institute for Experimental Medicine. They were housed with 2-5 animals per cage, on a 12 h / 12 h light / dark cycle with food and water ad libitum unless expressly stated otherwise. All experiments conducted have been approved by the German animal welfare law and the regulations of the State of Lower Saxony for animal experiments (LAVES).

The forebrain-specific conditional knockout lines lacking MBP were genotyped for three genes, the Cre driver, the floxed gene, and an ELLA2- Cre to make sure no other Cre was present except for the expected Cre driver. Genotyping was done from genomic DNA extracted from ear-punch biopsies, used to number experimental animals and running polymerase chain reaction (PCR) along with appropriate annealing primers. The MBPfl/fl. Generated by Dr. Wiebke Möbius in the Max Planck institute for Experimental Medicine was genotyped as described previously (Meschkat et al., 2020). The two forebrain-specific Cre drivers used have also been described previously. The Emx1Cre (Gorski et al., 2002) is a line of mice in which the Cre recombinase enzyme is expressed under the Emx1 promoter. The FoxG1Cre driver line is the same except the Cre recombinase is expressed under the FoxG1 promoter (Kawaguchi et al., 2016). 


\subsubsection{Biochemical Protein Quantification}

\subsubsection{Tissue isolation and sample preparation}

I sacrificed male mice of the appropriate genotype via cervical dislocation. I then excised out the brains cut and approximately $1 \mathrm{~mm}$ thick slices using a sagittal brain matrix. I dissected out the white matter, cortex and cerebellum from each slice and collected them on a glass plate placed on ice. I transferred the material into Eppendorf tubes, placed on dry ice. I stored these tubes at $-80^{\circ} \mathrm{C}$. For sample preparation, I transferred the tissue into Precellys tubes containing $500 \mu \mathrm{L}$ of modified RIPA buffer and homogenized it using the Precellys 24 (Peglab, Erlangen, Germany) with a program of $6000 \mathrm{rpm}, 2 \times 15 \mathrm{sec}$. I then centrifuged the samples at $13,000 \mathrm{rpm}$ for 10 minutes at $4^{\circ} \mathrm{C}$. The supernatant was transferred to fresh tubes and once again stored at $-80^{\circ} \mathrm{C}$.

To measure the protein concentration of the samples, I diluted (1:10) them and used the Bio-Rad DC protein assay kit as per manufacturer's instructions to measure the optical density at $650 \mathrm{~nm}$ using the Eon ${ }^{\mathrm{TM}}$ High Performance Microplate Spectrophotometer (BioTek, Vermont, USA). I prepared $200 \mu \mathrm{L}$ of loading samples containing $1 \times$ SDS sample buffer, $200 \mu \mathrm{g}$ of protein and $5 \% \beta$ mercaptoethanol $(\mathrm{v} / \mathrm{v})$. To denature the proteins, I incubated the samples at $95^{\circ} \mathrm{C}$, shaking at $300 \mathrm{rpm}$ for 10 minutes.

\subsubsection{SDS PAGE}

I prepared $15 \%$ polyacrylamide gels with a $5 \%$ stacking gel to separate proteins according to their electrophoretic mobility and size. I loaded $20 \mu \mathrm{g}$ of protein samples along with $3 \mu \mathrm{L}$ of pre-stained protein ladder (PageRuler ${ }^{\mathrm{TM}}$, Thermo Fischer Scientific). I ran the gels at a constant $120 \mathrm{~V}$ for 2 hours to ensure proper separation of my protein of interest, until the dye-front ran out of the gel.

\subsubsection{Western Blotting}

To perform Western blotting, I stacked the gel onto a PVDF membrane (PVDF Membrane Imobilon ${ }^{\circledR}$-FL P0.45 $\mu \mathrm{m}$, Merck Millipore) which I sandwiched between filter papers. I placed the sandwich in a BioRad WetBlot chamber 
containing chilled $1 \mathrm{x}$ transfer buffer (Table 6) and ran the transfer at $100 \mathrm{~V}$ for $1 \mathrm{~h} 15 \mathrm{~m}$. I then proceeded to wash the membrane in $1 \mathrm{x}$ TBST, two times for 5 minutes to get rid of any excess SDS. To detect the total amount of protein in each lane, I incubated the membrane in $0.1 \%$ Fast Green solution for 5 minutes, shaking at $300 \mathrm{rpm}$. I washed off the excess dye with Fast Green washing solution (Table 6), two times for 30 seconds each and imaged the membrane using the ECL Chemostar (Intas Science Imaging, Göttingen, Germany). This also acted as a loading control and so I could later normalize my protein of interest with it. I washed the membrane with TBST, two times for 5 minutes, ensuring all acetic acid was washed off. I then blocked the blots with $5 \%$ non-fat dry milk powder prepared in TBST, for 30 minutes rotating at room temperature (RT). I transferred the membranes in to falcons containing the requisite dilution of primary antibodies (Table 2) in $5 \%$ non-fat dry milk powder in TBST rotating at $4^{\circ} \mathrm{C}$ overnight. The next day, I washed the membrane three times for 5 minutes each in TBST to wash off excess primary antibody. I incubated the washed membrane in secondary antibodies also prepared in appropriate dilutions in $5 \%$ non-fat dry milk powder in TBST for 1 hour rotating at RT. I washed the excess secondary antibody with two 5 minute long washed in TBST and imaged the membranes using the LI-COR Odyssey CLx imaging system (LI-COR Biosciences $\mathrm{GmbH}$ ). I analyzed the Tagged Image File Format (TIFF) images using ImageJ (NIH) to find the relative abundance of my protein of interest in the white matter, cortex and cerebellum.

\subsubsection{Immunohistochemistry}

\subsubsection{Tissue Acquisition}

Male and female mice of appropriate ages and genotype were anaesthetized with i.p. injection of Avertin ( $0.2 \mathrm{~mL} / 10 \mathrm{~g}$ of body weight). They were then transcardially perfused with $5 \mathrm{~mL}$ of Hanks balanced salt solution (HBSS, PAA laboratories) and 4 \% PFA in $0.1 \mathrm{M}$ Phosphate buffer using a Heidolph PD5201 peristaltic pump. The brains were carefully excised out in post-fixed in $4 \%$ PFA for a further 2-hour period. All perfusions were carried out by Torben Ruhwedel and Andrew Octavian Sasmita of the Max Planck Institute for Experimental Medicine. 


\subsubsection{Paraffin Embedding of Brains for Immunohistochemistry}

The post-fixed brains were embedded in paraffin in an automated embedding machine (HMP 110, MICROM). The following protocol was used:

$50 \%(\mathrm{v} / \mathrm{v})$ Ethanol $1 \times 1 \mathrm{~h}$

$70 \%(\mathrm{v} / \mathrm{v})$ Ethanol $2 \times 2 \mathrm{~h}$

$96 \%(v / v)$ Ethanol $2 \times 2 h$

$100 \%(v / v)$ Ethanol 2x 2h

$100 \%(\mathrm{v} / \mathrm{v})$ Isopropanol $1 \times 1 \mathrm{~h}$

$100 \%(v / v)$ Xylol 2x 2h

$100 \%$ (v/v Paraffin $2 \times 2 h$

The infused samples were placed in metal forms and embedded into paraffin blocks which were cooled. The blocks were extracted from the metal forms and stored at RT until sectioning. Using a manual microtome (HM 400, MICROM), the brain blocks were cut into $5 \mu \mathrm{m}$ thick sections and collected on glass slides. 2-3 sections per slide were collected as replicates for analysis. The slides were dried overnight at $37^{\circ} \mathrm{C}$ and then stored at RT. All embedding and sectioning was done by Annette Fahrenholz.

\subsubsection{Immunohistochemistry of Paraffin-Embedded Sections}

Two kinds of immunohistochemical protocols were conducted on the paraffinembedded sections: Fluorescent and DAB labelling of sections.

Before the immunohistochemistry could be performed, the slices had to be deparaffinized to expose the epitopes to which the primary antibodies would bind. The protocol to do so was as follows:

Surrounding paraffin was melted at $60^{\circ} \mathrm{C} 10 \mathrm{~min}$

Xylol $100 \%$ 2x 10 min

Xylol: Isopropanol (1:1) $10 \mathrm{~min}$

Ethanol $100 \%(\mathrm{v} / \mathrm{v}) 5 \mathrm{~min}$

Ethanol $90 \%(\mathrm{v} / \mathrm{v}) 5 \mathrm{~min}$

Ethanol $70 \%(\mathrm{v} / \mathrm{v}) 5 \mathrm{~min}$

Ethanol $50 \%(\mathrm{v} / \mathrm{v}) 5 \mathrm{~min}$

$\mathrm{ddH}_{2} \mathrm{O} 5 \mathrm{~min}$ 


\section{Results}

To further improve the antibody binding capabilities, slides were transferred into glass cuvettes in a bath containing $0.1 \mathrm{M}, \mathrm{pH} 6.0$ citrate buffer boiled for $10 \mathrm{~min}$ (600W, microwave). The samples were allowed to cool for $20 \mathrm{~min}$.

For DAB labelling, slides were washed with $2 \%$ non-fat dry milk (w/v) powder in Tris buffer for $5 \mathrm{~min}$ and transferred to Shandon coverplates (Thermo Scientific). $100 \mu \mathrm{L}$ of $3 \%(\mathrm{v} / \mathrm{v}) \mathrm{H} 2 \mathrm{O} 2$ solution was added to each slide for $5 \mathrm{~min}$ to prevent endogenous peroxidases from converting $D A B$ substrate into nonspecific background signal. The sections were rinsed thrice with $2 \%$ non-fat dry milk (w/v) powder in Tris buffer and blocked with $20 \%(\mathrm{v} / \mathrm{v})$ goat serum in PBS/BSA for 10 to $30 \mathrm{~min}$ at RT. Primary antibodies (Table 1) were accordingly diluted in PBS/BSA and $100 \mu \mathrm{L}$ were applied to the coverplates and incubated at $4^{\circ} \mathrm{C}$ overnight. The next day, the slides were rinsed with $2 \%$ non-fat dry milk (w/v) powder in Tris buffer.

$100 \mu \mathrm{L}$ of biotinylated bridging antibody from the LSAB2 kit (Dako) was applied to the slides for $10 \mathrm{~min}$ at RT and rinsed with $2 \%$ non-fat dry milk (w/v) powder in Tris buffer. An HRP conjugated secondary antibody was then applied for 10 min at RT and washed off with $2 \%$ non-fat dry milk (w/v) powder in Tris buffer. 3,3'Diaminobenzidine (DAB) an HRP substrate was subsequently added using the DAB Zytomed kit (Zytomed Systems $\mathrm{GmbH}$ ) for $10 \mathrm{~min}$. DAB precipitated as brown residues on sites where antigen of interest was present. The slides were washed twice with $\mathrm{dd}_{2} \mathrm{O}$ for $5 \mathrm{~min}$ each and incubated for $5 \mathrm{~min}$ in $0.1 \%$ Haematoxylin for nuclear staining. After rinsing the slides with $\mathrm{ddH}_{2} \mathrm{O}$. twice, they were incubated shortly in HCL-Alcohol $(0.09 \% \mathrm{HCL}-70 \%$ Ethanol(v/v)). After another round of rinsing, they were incubated for $5 \mathrm{~min}$ in Scott's solution $(0.2 \%[\mathrm{w} / \mathrm{v}]$ Potassiumhydrogencarbonate $2 \%[\mathrm{w} / \mathrm{v}]$ Magnesium sulphate) and once again rinsed. The slides were incubated in $0.1 \%(\mathrm{w} / \mathrm{v})$ Eosin $(0.1 \%(\mathrm{v} / \mathrm{v})$ Eosin in $\mathrm{ddH} 20)$ for 3-5 min as counterstaining and rinsed in $\mathrm{ddH}_{2} \mathrm{O}$. The slides were serially dehydrated in increasing ethanol solutions for $5 \mathrm{~min}$ each, followed by Xylol:Isopropanol (1:1), 2x Xylol and mounted with Eukitt (EUKITT, o-kindler). In case of fluorescent labelling, the slides were incubated in $0.1 \%$ Triton in PBS for $15 \mathrm{~min}$ and washed once with PBS. They were once again fixed on to Shandon coverplates (Thermo Scientific) and $100 \mu \mathrm{L}$ of blocking solution containing $10 \%$ goat serum in PBS was added to the slides and incubated for 20 min at RT. 
Primary antibodies diluted appropriately in $10 \%$ goat serum and PBS was added on to the slides and incubated at $4^{\circ} \mathrm{C}$ overnight (Table 2). The next day, the slides were washed with PBS, thrice for 5 min each. Secondary antibodies (Table 2) with appropriate dilutions were prepared in $10 \%$ goat serum in PBS along with DAPI $(1: 10,000)$ for nuclear staining and applied to the slides. They were then incubated at RT for 2 hours in a cool and dark spot, to avoid bleaching of the conjugated fluorophores. Excess antibodies were washed off with PBS, thrice for 5 min each and mounted with Aqua-Poly/Mount (Polysciences) and stored at $4^{\circ} \mathrm{C}$. DAB immunohistochemistry was performed by Annette Fahrenholz.

\subsubsection{Imaging and Analysis}

DAB labeled slides were imaged using a Zeiss Axiolmager Z1 containing a Zeiss AxioCam MRc camera at 20x magnification using ZEN software by Jonas Hemesath, my master's student.

I imaged the fluorescently labeled slides using an Axio-Observer epifluorescence microscope (Zeiss) equipped with a Zeiss AxioCam MR R3 using ZEN 2012 software, at 20x magnification.

The following filter cubes were used for the respective fluorophores:

Alexa 555 dsRed

Alexa 633 Cy5

The GFP filter cube was used to record auto-fluorescence.

All images were processed and quantified using ImageJ and Zen 3.2 LITE. The data was visualized using GraphPad Prism 8.

\subsubsection{Electron Microscopy}

\subsubsection{Tissue Collection and Epon Embedding}

Male mice of appropriate age and genotype were anaesthetized and transcardially perfused with HBSS and Karlsson and Schultz fixative solution. $200 \mu \mathrm{m}$ thick saggital section were cute from the excised brains using a vibratome (VT1200S, Leica). Thr rostral region of the corpus callosum was isolated using a biopsy punch (harries Unicore $1.5 \mathrm{~mm}$, GE Healthcare). The punches were wrapped in tea filter paper and placed in plastic baskets (Science Services) and transferred into a Lynx 
Tissue Processor II (Science Services). Using the following protocol, these punches were embedded for electron microscopy:

\begin{tabular}{ccc} 
Reagent & Time & Temperature \\
\hline $0.1 \mathrm{M} \mathrm{PB}$ & $3 \times 10 \mathrm{~min}$ & $4^{\circ} \mathrm{C}$ \\
$2 \%$ OsO4 & $4 \mathrm{~h}$ & $4^{\circ} \mathrm{C}$ \\
$0.1 \mathrm{M} \mathrm{PB}$ & $3 \times 10 \mathrm{~min}$ & $4^{\circ} \mathrm{C}$ \\
$30 \%$ Acetone & $20 \mathrm{~min}$ & $\mathrm{RT}$ \\
$50 \%$ Acetone & $20 \mathrm{~min}$ & $\mathrm{RT}$ \\
$70 \%$ Acetone & $20 \mathrm{~min}$ & $\mathrm{RT}$ \\
$90 \%$ Acetone & $20 \mathrm{~min}$ & $\mathrm{RT}$ \\
$100 \%$ Acetone & $10 \mathrm{~min}$ & $\mathrm{RT}$ \\
$100 \%$ Acetone & $10 \mathrm{~min}$ & $\mathrm{RT}$ \\
$100 \%$ Acetone & $10 \mathrm{~min}$ & $\mathrm{RT}$ \\
$100 \%$ Acetone & $10 \mathrm{~min}$ & $\mathrm{RT}$ \\
Acetone /Epon $2: 1$ & $2 \mathrm{~h}$ & $\mathrm{RT}$ \\
Acetone /Epon $1: 1$ & $2 \mathrm{~h}$ & $\mathrm{RT}$ \\
Acetone /Epon $1: 2$ & $4 \mathrm{~h}$ & $\mathrm{RT}$ \\
Epon 100\% & $4 \mathrm{~h}$ & $\mathrm{RT}$
\end{tabular}

The dehydrated punches were embedded in gelatine capsules filled with Epon and allowed to polymerize at $60^{\circ} \mathrm{C}$ for $24 \mathrm{~h}$.

Epon-embedded samples were trimmed and cut into $500 \mathrm{~nm}$ semi thin sections using a RMC ATUMtome-Ultramicrotome (Science Services) or a Leica UC7 ultramicrotome (Leica) containing a DiATOME diamond knife, ultra $35^{\circ} 3.0 \mathrm{~mm}$ (Diatome). The sections were stained with methylene blue/azure II blue for 30-45 seconds to visualize lipid-rich regions on the tissue to confirm the region of interest (rostral corpus callosum). 50-60 nm ultra thin sections were then transferred to copper mesh grids (Science Services) and air-dried. The sections were contrasted with Uranyless for $30 \mathrm{~min}$ (Electron Microscopy Sciences, Hatfield, U.S.A) and washed 5 times with $\mathrm{ddH}_{2} \mathrm{O}$. Analysis of the sections was done with a LEO EM912 Omega (Zeiss, Oberkochen, Germany), and digital electron micrographs were captured with a wide-angle dual speed 2K-CCD-camera (TRS, Moorenweis, 


\section{Results}

Germany) using the microscope software iTem (Olympus) and ImageSP (SysProg).

\subsubsection{Image Analysis}

All image analysis was done in ImageJ $(\mathrm{NIH})$. Transmission electron microscopy images of the rostral corpus callosum ( $\mathrm{rCC}$ ) were quantified for the total number of normally and abnormally (shiverer-type) myelinated axons i.e. axons with noncompacted myelin surrounding them. Three randomly selected images per animal were analyzed to calculate the mean count of axons exhibiting the shiverer phenotype.

For axons that were normally myelinated, g-ratios were calculated. Random selection of axons was mediated via projecting a grid on the images and axons at the intersecting gridlines were selected. Cross sections that shows any sign of sectioning artefacts were not considered for analysis. Thus, the outer border of the myelin sheath $\left(A_{0}\right)$ and the axonal plasma membrane $\left(A_{i}\right)$ are was measured and the outer fimber diameter $\left(d_{o}\right)$ and the axon diameter $\left(d_{i}\right)$ were calculated using the following equation:

\section{Equation 1: Calculation of axon diameter}

$$
d_{x}=2 X \sqrt{\frac{A_{x}}{\pi}}
$$

The g-ratio is given by:

\section{Equation 2: Calculation of g-ratio}

$$
\mathrm{g}-\text { ratio }=\frac{d_{i}}{d_{o}}
$$

For shiverer-like axons, only the axon calibre was assessed.

All image acquisition and analysis was performed on ImageJ (NIH) and using RStudio (RStudio Inc.) by Jonas Hemesath and included in his master's thesis. 


\subsubsection{Paw Preference Test}

\subsubsection{Behavioral paradigm}

I adapted the behavioural paradigm developed by Richard L. Collins (Collins, 1968) to assess the inheritance of handedness in different strains of laboratory mice. To elucidate the critical period of paw lateralization in different myelin mutants, I modified the test (Figure 6 A). I used both male and female mice since they didn't show any difference in behaviour with respect to the paradigm.

The entire paradigm begins with weighing the mice three days prior to the training session. To prevent neophobia, which is the fear of new smells, often observed in mice I weighed them every day and provided 10 pellets (TSE precision pellets, 14 $\mathrm{mg}$ ) per mouse in a cage. The evening before training day, I restricted the amount of food to the mice ( $0.08 \mathrm{~g} / \mathrm{g}$ of weight) (Figure $6 \mathrm{~B}$ ). Water was provided ad libitum I used a custom-made Plexiglas box with the following dimensions: $10 \mathrm{~cm}$ long $\mathrm{x}$ $10 \mathrm{~cm}$ wide $\times 12 \mathrm{~cm}$ deep. A feeding tube, $8 \mathrm{~mm}$ in diameter, was placed $2.6 \mathrm{~cm}$ from the base.

On training day, I allowed for mice to habituate in the behaviour room for at least 30 minutes. I weighed the mouse and placed in the box and allowed to habituate it for 10 minutes (Table 7: Habituation and retrieval times for the paw preference paradigm). A few minutes into the habituation, I pushed a pellet through the food tube, so the mice would know where the food comes from. I then placed a second pellet at the very edge of the tube to motivate it to retrieve the pellet on its own. After the 10 minutes of habituation had lapsed, I started recording the retrieval session with a video camera (Sony Handycam DCR-SR57 Carl Zeiss Vario Tessar, $25 \mathrm{ppm}$ ). The retrieval session on either day lasted for up to 20 minutes or until 25 pellets were successfully retrieved. I placed the pellet approximately $0.5 \mathrm{~cm}$ away from the tube edge to ensure the camera or I recorded the paw the mouse used to retrieve the pellet. After the retrieval I weight the mice once more and provided the restricted amount of food ( $0.1 \mathrm{~g}$ per $\mathrm{g}$ of weight), plus 10 pellets per mouse, per cage. 
A

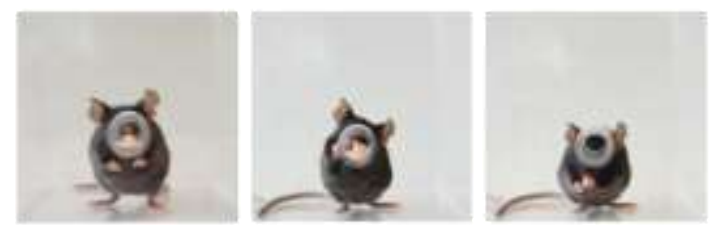

B

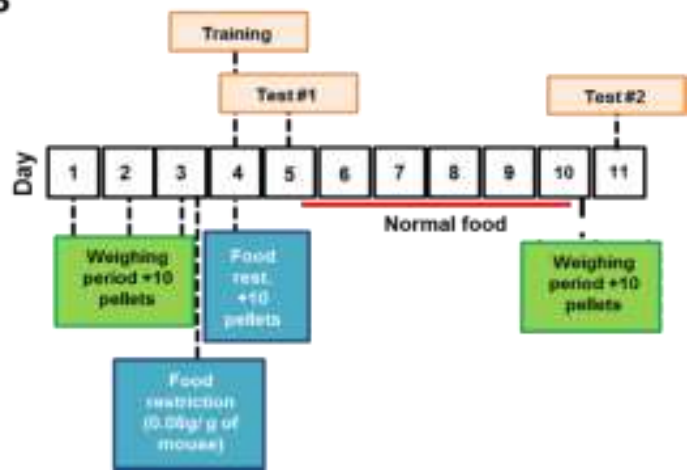

Figure 6: The modified paw preference paradigm.

A) A mouse performing pellet retrieval during a paw preference test. Beginning with the decision to retrieve, the mouse reaches for the pellet, followed by successfully retrieving the pellet (left to right).

B) The modified test protocol. Mice are weighed three days prior to the first training session, to avoid effects of neophobia and the evening before training, they undergo food restriction $(0.08 \mathrm{~g}$ of food/ $\mathrm{g}$ of weight). Mice are continued on the restricted diet on test \#1 day and are provided food ad libitum after the retrieval session. Water is always provided ad libitum. Pictures provided by Dr. Sharlen Moore.

The day of Test\#1, I habituated the mice between 3 - 5 minutes. The rest of the behavioural test was the same as training day. At the end of this retrieval, I gave the mice normal amount of food. I considered the test sessions for statistical analysis.

For repetitively tested mice, I weighed them every week before the test and restricted their food $(0.08 \mathrm{~g}$ food per $\mathrm{g}$ of mouse).

Table 7: Habituation and retrieval times for the paw preference paradigm

\begin{tabular}{ccc} 
Test & Habituation time & Retrieval time \\
\hline Training & 10 minutes & 20 minutes \\
Test\#1 & $3-5$ minutes & 20 minutes
\end{tabular}

\subsubsection{Analysing Lateralization in Mice}

Within the test session, I manually counted the number of right paw entries (\#RPE) and left paw entries (\#LPE). Attempts with both paws were considered as one 


\section{Results}

ambilateral attempt and taken into account in the total paw entries (\#TPE). For a mouse to qualify for analysis, it had to make $\geq 10$ total attempts. This was important to gauge reliably whether a mouse was truly left-pawed or right-pawed. The following equation was used to calculate \#TPE:

\section{Equation 3: Total Paw Entries}

$$
\# \mathrm{TPE}=\# \mathrm{RPE}+\# \mathrm{LPE}
$$

To determine whether a mouse was left-pawed or right-pawed in a given test session, I calculated the \% RPE using the following equation:

\section{Equation 4: Direction of Handedness}

$$
\text { Direction of Handedness }(\% \text { RPE })=\frac{\# R P E}{\# T P E} \times 100
$$

A \% RPE value of 0 corresponded with a left-pawed mouse whereas 100 corresponded with strongly right-pawed mice. A value of 50 meant that the mouse was ambilateral with respect to the behavioural paradigm. To assess the extent of lateralization of a mouse, I calculated the strength of lateralization using the following equation:

\section{Equation 5: Strength of Lateralization}

$$
\text { Strength of Lateralization }(|\mathrm{PPE}|)=\mathrm{ABS}\left(\frac{\# \mathrm{RPE}-\# \mathrm{LPE}}{\# \mathrm{TPE}} \mathrm{X} 100\right)
$$

Here, a value of 0 corresponded with an ambilateral mouse and a mouse having a value of 100 was considered to be fully lateralized, i.e. having a single paw preference. I set an arbitrary threshold at 75 , meaning mice showing a strength of lateralization $\geq 75$ were considered to be lateralized.

Mice that showed a drastic change in their handedness had to be assessed for their instability. This was done with the following equation:

\section{Equation 6: Instability Index}

$$
\text { Instability Index }=\mathrm{ABS}\left(\frac{\% \mathrm{RPE}_{4}-\% \mathrm{RPE}_{8}}{100}\right)
$$


The instability index was calculated between the performance of the mice at 4 and 8 weeks to investigate the effects of repetitive training. An instability index of 0 meant the mouse was very stable in its handedness and consistent across all test sessions. Once again, I applied an arbitrary threshold of 0.2, i.e. a mouse showing an instability index $>0.2$ was considered unstable.

A relative distribution of the population of mice that were right-pawed or left-pawed was generated by binning their direction of handedness in 10 bins between $0-100$. A chi-square test was conducted on this distribution to see the divergence of the mutant mice from control littermates. The confidence level was $95 \%$.

\subsubsection{Behavioural Assessment of Higher-Brain Functions}

To assess whether there was a cognitive advantage or deficit in the forebrainspecific knockout of MBP, a large cohort of both male and female, mutant and control mice were examined by a myriad of behavioural tests. These experiments were conducted by Sahab Arinrad and Anja Ronnenberg of the Max Planck Institute for Experimental Medicine. I conducted the analysis.

\subsubsection{Mouse Maintenance and Behavioural Tests}

All mice were housed as mentioned above in section 2.2.1. The only difference here was that mice were separated into group housing based on their gender and genotype, upon weaning. Once they were 6 months old, male mice were single housed for distinct experimental reasons. Female and male conditional knockout (cKO) mice ( $\mathrm{N}=11$ and 19 , respectively) were compared to control (CTL) mice ( $N$ $=17$ and 23, respectively) for the following behavioural experiments. A set of behavioural tests were conducted between the ages of 2-23 months. A summary of the results of the different experiments, number of mice, age of the mice, mean, SEM and $p$-values are given in Table 9 and Table 10 . outliers were tested for using the online GraphPad Grubbs outlier tool. Mice of both gender ad genotypes that didn't perform were excluded from statistical analyses. Habituation to the conditions was done for 30 minutes prior to testing in the experimental room. Female and male cohorts were tested separately. A summary of the behavioural experiments conducted on the mice at specific age time-points is given in Table 8. 


\section{Results}

Table 8: Summary of different behavioural domains and the corresponding tests conducted to evaluate Emx1Cre/Mbp flox mice

\begin{tabular}{|c|c|c|c|}
\hline Behavioral domai & $\begin{array}{c}\text { Test } \\
\text { Paradigm }\end{array}$ & $\begin{array}{l}\text { Males } \\
\text { (age in } \\
\text { months) }\end{array}$ & $\begin{array}{l}\text { Females } \\
\text { (age in } \\
\text { months) }\end{array}$ \\
\hline Health status & Body weight & throughout & throughout \\
\hline \multicolumn{4}{|l|}{ Anxiety } \\
\hline Anxiety-like behavior & Elevated plus maze & 2 & 3 \\
\hline Anxiety-like behavior & Open field & 2 & 3 \\
\hline Anxiety-like behavior & Light dark box & 3 & 3 \\
\hline \multicolumn{4}{|l|}{$\begin{array}{l}\text { Motor performance } \\
\text { and exploration }\end{array}$} \\
\hline Explorative behavior & Hole-board & $3-4$ & $3-4$ \\
\hline Motor learning & Rotarod & $3-5$ & $3-5$ \\
\hline Muscle strength & Grip strength & $3-5$ & $3-5$ \\
\hline Home-cage behavior & LABORAS & 10 & 9 \\
\hline \multicolumn{4}{|l|}{$\begin{array}{c}\text { Sensory } \\
\text { performance }\end{array}$} \\
\hline Hearing & $\begin{array}{c}\text { Acoustic startle } \\
\text { response }\end{array}$ & $3-19$ & $4-19$ \\
\hline Vision & Visible days MWM & 3 & $5-6$ \\
\hline \multicolumn{4}{|l|}{$\begin{array}{c}\text { Cortical network } \\
\text { function }\end{array}$} \\
\hline Sensorimotor gating & Pre-pulse inhibition & $3-19$ & $4-19$ \\
\hline \multicolumn{4}{|l|}{ Social performance } \\
\hline Social preference & Tripartite chamber & $3-4$ & $6-7$ \\
\hline Social memory & Tripartite chamber & $3-4$ & $6-7$ \\
\hline \multicolumn{4}{|l|}{$\begin{array}{l}\text { Catatonia and } \\
\text { executive function }\end{array}$} \\
\hline Catatonic signs & Bar test & $2-17$ & $3-17$ \\
\hline Executive function & Hurdle test & $3-17$ & $3-17$ \\
\hline Executive function & Puzzle box & $4-18$ & $5-18$ \\
\hline
\end{tabular}


Cognition

Spatial memory
Morris water maze
3

$5-6$

\subsubsection{Cage Behaviour and Health Evaluation}

The health of the mice was monitored throughout. Home-cage behaviour, interaction with cage mates, activity of the mice were a few parameters that were closely observed. In addition, the body weight of all mice was monitored throughout the paradigms.

\subsubsection{Anxiety - Like Behaviour}

\subsection{Elevated Plus Maze}

The elevated plus maze $(5 \mathrm{~cm} \times 5 \mathrm{~cm}$ central platform, made of grey Perspex, with two walled and two open arms each $30 \mathrm{~cm} \times 5 \mathrm{~cm} \times 15 \mathrm{~cm}$ in dimensions. The mouse was placed on the central platform facing an open arm and its behaviour was recorded for a 5 minute period, using an automated tracking software (Viewer2, Biobserve) and an overhead camera to calculate how much time the mouse spent in open arms vs the walled arms as an output for anxiety-like behaviour.

\subsection{Open Field Test}

The field in question consisted of a circular chamber $(120 \mathrm{~cm}$ in diameter and wall of $25 \mathrm{~cm}$ in height). The mouse was placed into the centre of the field for 7 minutes and its explorative behaviour was on recorded using a tracking software (Viewer2, Biobserve). Anxiety behaviour was assessed based on the total distance travelled by the animals and the amount of time it spent in the central, intermediate and peripheral zones.

\subsection{Light/Dark Box}

The apparatus which was $36 \mathrm{~cm}$ X $20.5 \mathrm{~cm}$ X $19 \mathrm{~cm}$ ) in dimensions, had two equal acrylic compartments. One that was dark, roofed and a white one with a light intensity of $300 \mathrm{~lx}$. A divider with a small opening $(5.7 \mathrm{~cm} \times 5 \mathrm{~cm})$ separated both compartments. The test began with the mouse being placed in the dark 
compartment, facing the white compartment and allowing the mouse to explore the environment for 5 minutes. The movement of the mouse from one side to the other and the total amount of time spent in the lit compartment was recorded. This method allowed one to assess the natural tendency of mice to stay in dark, covered places versus the natural drive to explore new environments. The more anxious a mouse, the less explorative it would be or vice versa.

\subsubsection{Motor Performance and Exploration}

\subsection{Hole-board Test - Explorative Behaviour}

The hole-board was a transparent box $(50 \mathrm{~cm} \times 50 \mathrm{~cm} \mathrm{X} 36 \mathrm{~cm})$ with an opaque board with 16 evenly spaced holes $(2.2 \mathrm{~cm}$ in diameter), $3 \mathrm{~cm}$ above the base of the box. The mouse was placed in the centre of the box and allowed to explore for 5 minutes. The number of nose-pokes were recorded by two layers of infrared beams measures by the AKS software (TSE Systems).

\subsection{Rotarod - Motor Coordination and Learning}

The rotarod (ENV-577M, Med Associates Inc. Georgia) was performed as previously described (Dere et al., 2014). Two trials over two consecutive days were conducted for the male and female cohorts. The trial lasted for up to 5 minutes during which mice had to run continuously with the speed of the rod accelerating from $4-40 \mathrm{rpm}$. The latency pf falls were evaluated during both trials and analysed.

\subsection{Grip Strength - Forelimb Muscle Strength}

A total of three consecutive trials were assessed for each mouse. The mouse was gently lifted by its tail and allowed to grasp a wire connected to a grip strength meter (TSE Systems). Once the mouse had gripped the wire, it was brought into a horizontal position and gently pulled back to assess its grip strength. The average of the three trails per mouse was considered for analysis. 


\subsection{LABORAS - Home-cage Behaviour}

The LABORAS system consisting of a triangular sensor platform (Carbon Fibre Plate $1000 \mathrm{~mm} \times 700 \mathrm{~mm} \times 700 \mathrm{~mm} \times 30 \mathrm{~mm}$, Metris B.V.), positioned on two orthogonally placed force transducers (Single Point Load Cells) and a third fixed point attached to a heavy bottom plate called a Corian Plate $(980 \mathrm{~mm} \times 695 \mathrm{~mm} \times 695 \mathrm{~mm} \times 48 \mathrm{~mm}$ stands on three adjustable spikes and can absorb external vibrations. Mice were housed in clear polycarbonate cages (Makrolon type II cage, $22 \mathrm{~cm} \times 16 \mathrm{~cm} \times 14 \mathrm{~cm}$ ) with a wood-chip bedding covered floor. The cage was placed directly on to the sensing platform, with the upper part of the cage (including the top, food hopper, and drinking bottle) suspended in a height-adjustable frame separate from the sensing platform. The mechanical vibrations caused by the movement of the animal result in electrical signals that were transformed by each force transducer, amplified to a fixed signal range, filtered to eliminate noise, digitized, and stored on a computer. The computer then processed the stored data using several signal analysis techniques to classify the signals into the behavioural categories of eating, drinking, scratching, circling, climbing, immobility, locomotion, and grooming. The dominating behaviour was scored. This Spontaneous behaviour was assessed from 17:00 until 09:00, with a $1 \mathrm{~h}$ habituation to the cages recording was initiated (Dere et al., 2014).

\subsubsection{Sensory Performance}

\subsection{Vision - Cued Platform Training via the Morris Water Maze}

The experimental protocol for the Morris water maze has been escribed before (Dere et al., 2014). To evaluate general vision in mice, the water maze consisted of a escape platform $1 \mathrm{~cm}$ below the surface of opaque water. A blue flag placed in the centre of this platform was used as a cue for locating the platform. To analyse whether mice could locate this platform from the cue, the data from two consecutive days of acquisition training were looked at. Each day, consisted of 4 trials with 5 minutes inter-trial-interval (ITI). A video-tracking system (Viewer3, Biobserve) helped record the escape latency, velocity of swimming and the path length. Average escape latency on each day was analysed as the output. 


\subsection{Hearing - Acoustic Startle Response}

The pre-pulse inhibition (PPI) setup allowed assessment of general hearing of mice in addition to cortical network function. A startle response evoked by an acoustic stimulus $(120 \mathrm{~dB})$ on a force-sensitive platform in comparison to a 2 minute habituation period of a $65 \mathrm{~dB}$ background noise (white noise) and six pulsealone trials with a startle stimulus of $120 \mathrm{~dB}$ for a $40 \mathrm{~ms}$ duration. A lowered startle response corresponded with a hearing disability in the mice.

\subsubsection{Cortical Network Function}

\subsection{Pre-pulse Inhibition - Sensorimotor Gating}

A small metal cage ( $82 \mathrm{~mm} \times 40 \mathrm{~mm} \times 40 \mathrm{~mm}$ ) was used to limit major movements and exploratory behaviour. A moveable platform on the base of this cage was attached to a sensor that records vertical movement of the floor. The cage was placed in an attenuated cabinet (TSE Systems) and startle reflexes were recoded over a period of $260 \mathrm{~ms}$, beginning with the onset of the pulse. With a $2 \mathrm{~min}$ habituation consisting of a $65 \mathrm{~dB}$ white noise as background played throughout, a $1 \mathrm{~min}$ baseline recording followed. Then six pulse-only startle stimuli of $120 \mathrm{~dB}$ were applied for $40 \mathrm{~ms}$. To test for PPI, a startle pulse was applied alone or after a $20 \mathrm{~ms}$ long stimulus of 70,75 or $80 \mathrm{~dB}$. A delay of $100 \mathrm{~ms}$ with background noise was interspersed between the pre-pulse and pulse stimulus. The trials presented were in a pseudorandom order with a variable interval ranging from 8 to $22 \mathrm{~s}$. The amplitude of the startle response (expressed in arbitrary units) was defined as the difference between the maximum force detected during the recording window and the force measured immediately before the stimulus onset (Dere et al., 2014). the amplitudes were averaged separately for the two types of trials (i.e., stimulus alone or stimulus preceded by a pre-pulse). PPI was calculated as the percentage of the startle response using the following formula:

\section{Equation 7: Percentage PPI}

$$
\% \text { PPI }=100-\left[\frac{\text { startle amplitude after pre }- \text { pulse }}{\text { startle amplitude after pulse only }} \mathrm{X} 100\right]
$$




\subsubsection{Social Performance}

\subsection{Social Preference and Memory - Tripartite Chamber}

The tripartite chamber has been elucidated in detail previously (Dere et al., 2014). As the name goes, the experimental setup consists of three chambers. The test mouse was placed in the middle chamber and allowed to habituate for $5 \mathrm{~min}$. In an alternating manner, a stranger mouse was introduced to the two outer chambers in wire cages. The dividers between the chambers were opened and the test mouse was allowed to explore for $10 \mathrm{~min}$. Movement was tracked via video (Viewer2, Biobserver). In the third trial a new stranger mouse and the first stranger mouse were placed in the outer chambers and the dividers were removed. Intact social memory was indicative then the test mouse chose to explore the new stranger mouse. Sociability or social memory index was calculated as follows:

\section{Equation 8: Sociability Index}

Sociability Index $=\left(\frac{\text { time investigating stranger }}{\text { time investigating stranger }+ \text { time investigating empty cage }}\right) \times 100$

\section{Equation 9: Social Memory Index}

Social Memory Index $=\left(\frac{\text { time investigating unfamiliar mouse }}{\text { time investigating unfamiliar }+ \text { familair mouse }}\right) \times 100$

\subsubsection{Catatonia and Executive Function}

\subsection{Catatonic Signs - Bar Test}

Symptoms for catatonia were assessed using the bar test (Janova et al., 2018). Mice were placed close to a metal horizontal bar until the mouse grabbed it by its paws. The mouse was allowed to stay in the position, standing upright, until it let go of the bar. Two consecutive trials were conducted at different time-points and they were recorded using a high-resolution camera (Sony HDR-CX405, Sony). The time spent immobile on the bar was used for analysis. 


\subsection{Executive Function - Modified Hurdle Test}

Executive function tests for the ability to make decisions. The hurdle box set up consisted of a circular open field with a polyvinylchloride comb inset built with crisscrossing connected combs $(5 \mathrm{~cm} \times 5 \mathrm{~cm})$ and $140 \mathrm{~lx}$ light intensity in the centre. The mice were placed in the centre and the time taken by them to reach the periphery or until $5 \mathrm{~min}$ had lapsed, was measured and recorded using a tracking software (Viewer3, Biobserver). Two trials were conducted, with a 5 min ITI. Executive function was calculated as the ratio of latency to the periphery (s) and the number of hurdles crossed (\#). For those mice that never crossed any hurdles, the ratio was modified as [(s) / (\#+1)].

\subsection{Executive Function - Puzzle Box}

The setup of the puzzle box consisted of rectangular arena $(75 \mathrm{~cm} \times 28 \mathrm{~cm} \times 25$ $\mathrm{cm})$ split into two, one closed off and one illuminated (140 Ix), with a small doorway (4 cm width). Over 5 consecutive days of testing, mice were required to overcome 5 different challenges of increasing difficulty, to reach the shelter via the doorway. The following challenges were applied:

Open doorway

Gateway within doorway

Gateway filled with bedding

Plug made of paper tissue

Plug made of nesting material

Three trials per day were conducted for each mouse in which they were exposed to two challenges daily. The duration of trials were increased for the final two challenges to ensure the mice had sufficient time to escape. Their performance was recorded via a tracking software (Viewer3, Biobserver) and the average escape latency was measured for each challenge and analysed.

\subsubsection{Spatial Memory - Morris Water Maze Test}

The Morris water maze test was conducted as described previously (Dere et al., 2014). To assess spatial memory, the blue flag used to evaluate general vision (2.2.6.5.1) was removed and the location of the hidden platform was relocated and the mice were tested for 8 days during hidden platform training. During this period 


\section{Results}

the mice were required to memorize the spatial information about the escape platform. During the training phases, mice underwent 4 trials with an ITI of 5 min. a probe test followed and the time spent in the target quadrant and the latency to target were used for analysis. To investigate cognitive flexibility, a reversal learning task was performed. The procedure was identical to the one used for the hidden platform training except that the escape platform was moved from the original position to a different quadrant. Reversal learning was tested for 4 days followed once again by a probe trial.

\subsubsection{Statistical Analysis}

Analysis and visualization of acquired data was done with Excel 2016 and GraphPad Prism 8. All acquired data was first tested for normal distribution using the Shapiro-Wilk test. For data that was normally distributed, an unpaired, twotailed t-test was performed for one-on-one comparisons and an analysis of variance (ANOVA) for multiple comparisons. For non-normally distributed data, a Mann-Whitney test was used to compare one-on-one data and a Kruskal Wallis test for multiple comparison. For relative distributions, a chi-square test was done. Significant values were expressed as n.s. not significant, ${ }^{*} p<0.05,{ }^{* *} p<.0 .01,{ }^{* * *}$ $\mathrm{p}<0.001$. 


\section{Results}

\subsection{Young wild-type mice appear to be lateralized by 8 weeks of age}

Critical periods for different cortical systems such as visual (Gordon and Stryker, 1996; Levelt and Hübener, 2012) and auditory systems (de Villers-Sidani et al., 2007; Popescu and Polley, 2010; Zhang et al., 2001) have been previously outlined in rodents. Since, myelination coincides with these brief windows of experiencedependent plasticity (Tau and Peterson, 2010) and myelination has been shown to regulate critical periods (McGee et al., 2005; Stephany et al., 2016), I wondered if there is a critical period for the establishment of paw preference in mice. I tested young, female, wild-type C57BL/6J mice repetitively between the ages of 4 and 8 weeks. I implemented a modified version of the paw preference behavioural paradigm (as elaborated in section 2.2.5 Paw Preference Test) to assess two aspects of handedness, the strength (extent of single paw usage; Equation 5) and directionality (whether they prefer the right or the left paw; Equation 4). Briefly, I placed a previously food-restricted mouse in a transparent box with a feeding-tube on the frontal wall and calculated the paw entries it made to retrieve a food pellet. The pellet was placed, in the tube, at a distance so that only one paw at a time could be inserted for retrieval. I tested the mice for two consecutive days at 4 weeks of age. The first day was a 'training' day to ensure they were familiar with the environment and the paradigm. The second day was the 'test' day and the scores from this day were used for the analysis. After this, I tested them once every week until 8 weeks of age (Figure 7 A). To avoid bias, I excluded all mice that performed less than 10 total paw entries per session, from analysis. I only further tested the mice that performed more than 10 trials on 'test' day, in the next weeks. I set an arbitrary threshold at the laterality score of $75 \%$, above which I considered the mice to be lateralized. Wild-type mice show a gradual increase in the strength of lateralization and appear to be completely lateralized (100\%) by 8 weeks of age $(p=0.0103)$ (Figure 7 B). This was furthermore apparent when I compared their laterality scores at 4 and 8 weeks of age $(p=0.0008)$ (Figure $7 \mathrm{C})$. 


\section{Results}

Mice from the C57BL/6J strain show a U-shaped distribution with respect to handedness, i.e. they tend to be either left or right-handed (Collins, 1975). I traced the establishment of either right or left-handedness in my mice and found this to be true at the 8-week time-point (Figure $8 \mathbf{A}$ ). When I calculated the relative frequency distribution of handedness at the 4- and 8-week time-points, I noticed that the same mice that were ambilateral, meaning they showed no strong preference for either paw, appeared to have a clear preference by the 8-week timepoint (Figure $8 \mathbf{B}$ ).

With these data, I concluded that the establishment of paw laterality in wild-type C57BL/6J mice occurs between 4 and 8 weeks of age. By then, they exhibit a 5050 distribution of handedness with $50 \%$ being right- and $50 \%$ being left-handed. Since interhemispheric transfer of information is involved in the establishment of handedness (Preuss et al., 2002), does the CC play a role in the establishment of handedness? Does the fact that it is heavily myelinated, further influence which paw is preferred? 
A

\begin{tabular}{|l|c|c|c|c|c|}
4 & 5 weeks & weeks & 6 weeks & weeks & weeks \\
\hline Training & Test &
\end{tabular}

B

Strength of Lateralization

C Strength of Lateralization
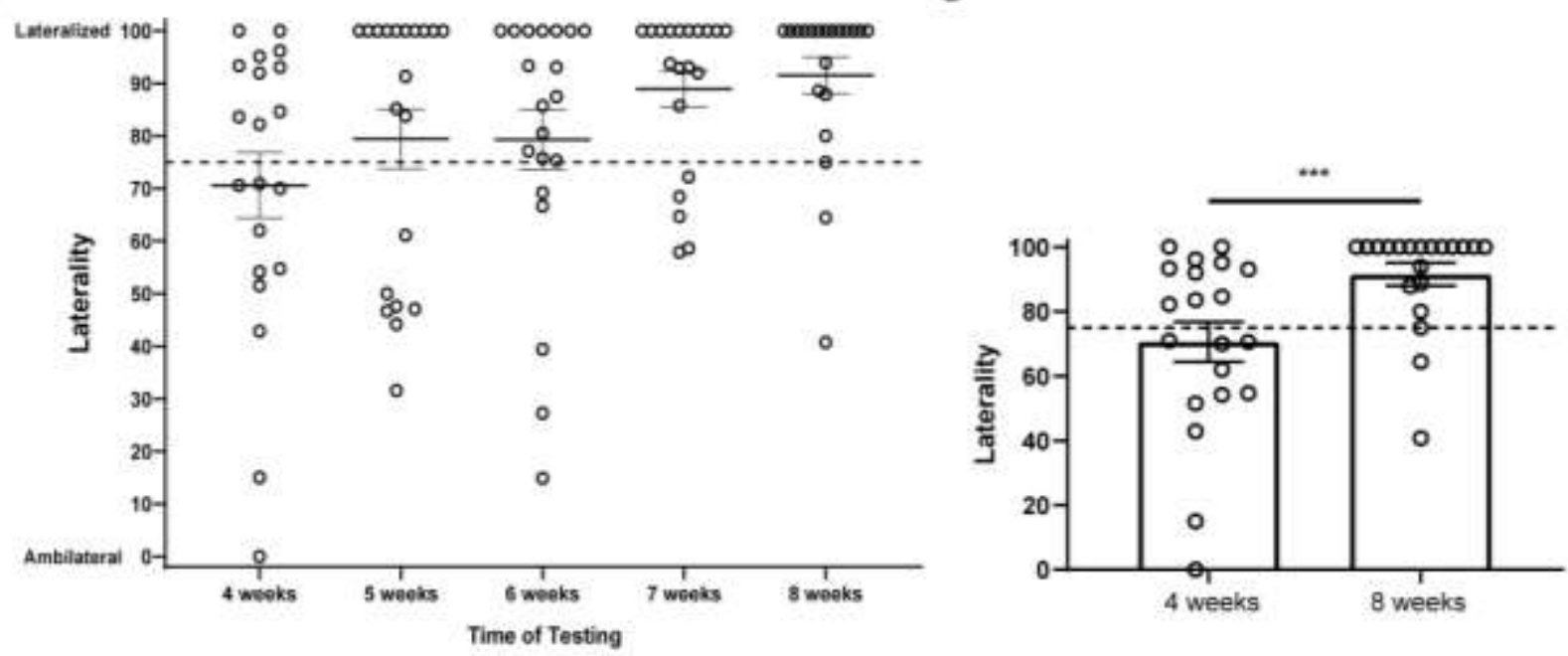

Figure 7: Establishment of behavioral laterality in young C57BL/6J mice.

A) Outline of the behavioral paradigm used to assess the establishment of behavioral laterality in young female C57BL/6J. They were tested repetitively on a weekly basis between 4 and 8 weeks of age. B) Strength of lateralization of the tested mice at all timepoints shows the progressive increase in it until 8 weeks $(n=20),{ }^{*}(p$-value $=0.0103)$, repeated measures one-way analysis of variance (ANOVA) with Tukey's multiple comparison test. C) Comparison between the 4- and 8-week time-points shows a significant increase in strength of lateralization, ${ }^{* * *}(p$-value $=0.0008)$, Mann-Whitney test. Laterality values, $0 \%=$ Ambilateral, $100 \%=$ Lateralized. Mean \pm S.E.M. (error bars). 
A

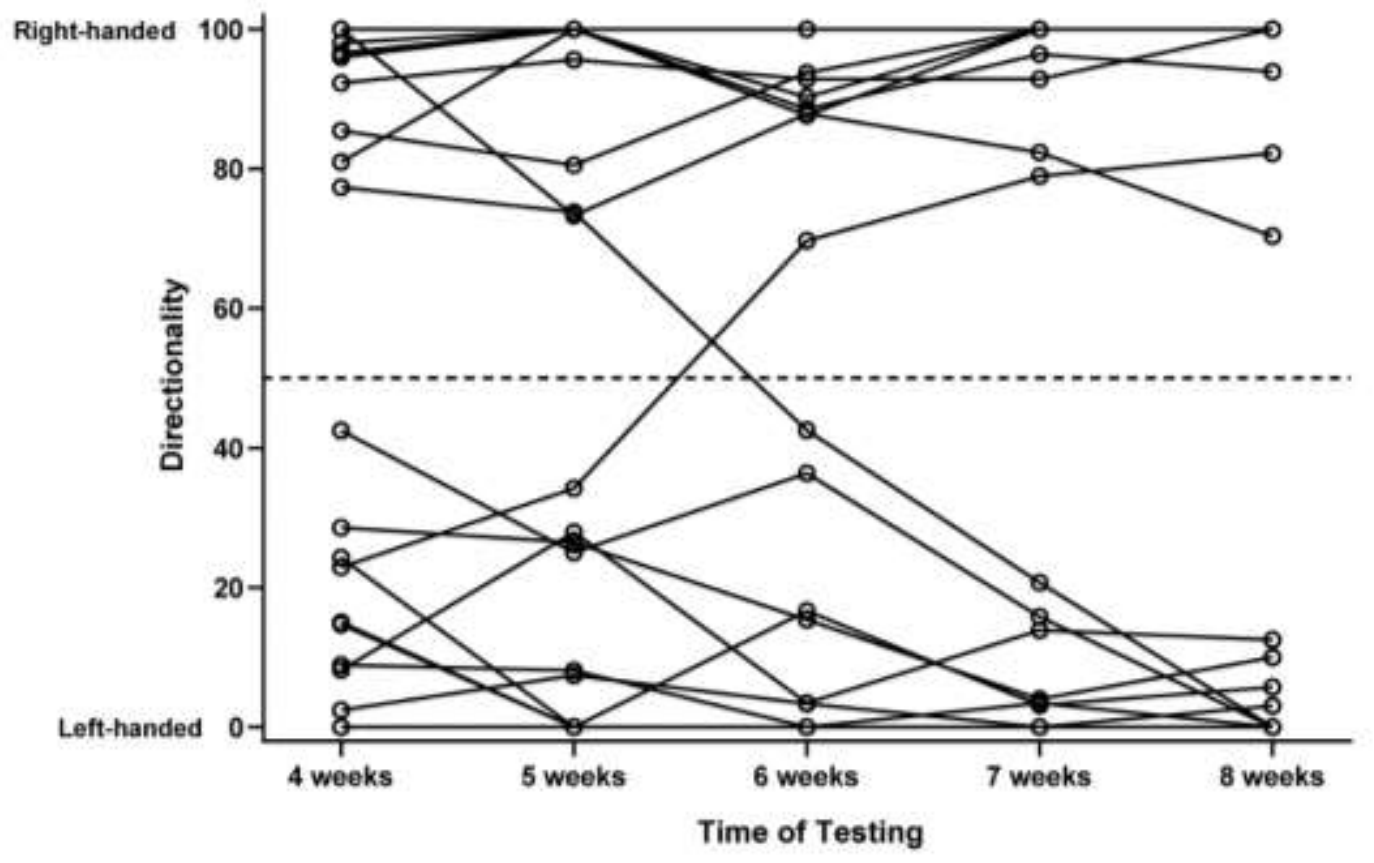

B

4 weeks

8 weeks
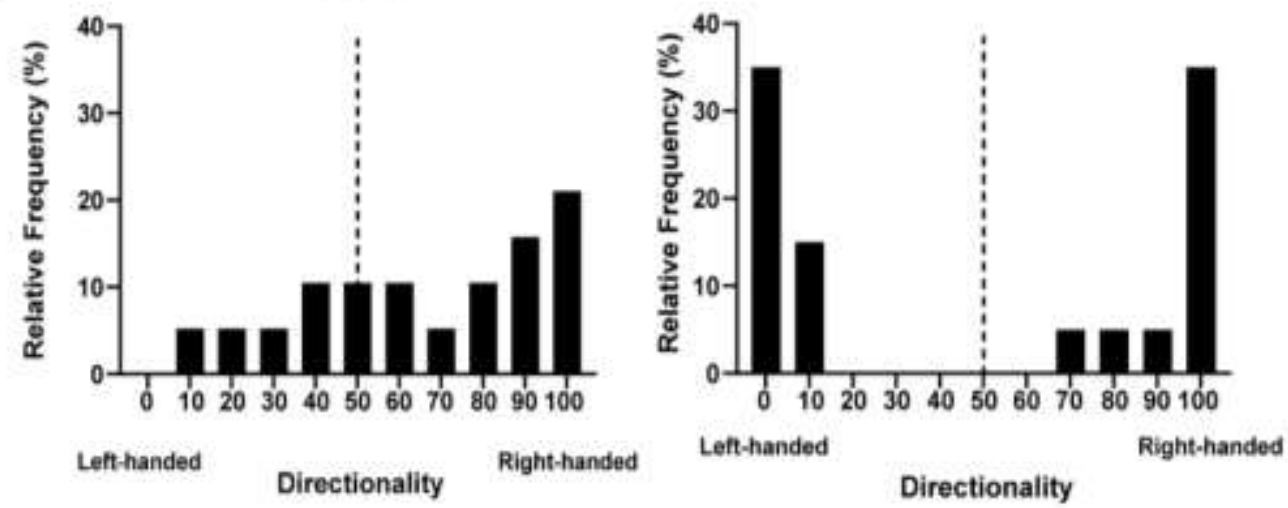

Figure 8: Lateralized wild-type mice show a U-shaped population distribution of handedness.

A) Directionality of individual mice tracked over the course of the behavioral paradigm reveals that each mouse that appears to be more ambilateral at the four-week timepoint was either right-handed or left-handed by eight weeks of age. B) Relative distribution of mice at four and eight-week time-points show that eight-week old wildtype mice show a stronger rightward or leftward bias culminating in a U-shaped distribution curve, which is characteristic of C57BL/6J mice (Collins, 1975). Directionality values, $0 \%=$ Left-handed, $50 \%=$ Ambilateral, $100 \%=$ right-handed. Bin size $=10$. 


\subsection{Young mice lacking compact myelin become lateralized with repetitive testing}

Myelination in mice begins after birth and peaks approximately at 3-4 weeks of age (Koch et al., 2008; Sauvageot and Stiles, 2002; Wright et al., 2010). Since it coincides with the proposed critical period for establishment of behavioural laterality, I wanted to investigate the role it might play in the process. For this, I turned to the shiverer mouse model (elaborated in section 1.1.3 The Myelin Basic Protein (MBP) is a Key Component of Compact Myelin). The mouse model is central to the research of demyelinating diseases such as multiple sclerosis (Joshi et al., 2015). I tested both female and male, young shiverer mice along with their wild-type littermates as control between the 4 and 9 weeks of age, on a weekly basis (Figure 9 A). For simplicity, I will refer to this cohort as "weekly". Shiverer mice appeared to become comparably lateralized by 9 weeks of age ( $p$-value $=$ 0.5978) (Figure $9 \mathrm{D}$ ), even though they showed significantly reduced laterality at the 4-5 week time-point ( $p$-value $=0.0306$ ) (Figure $9 \mathrm{C}$ ). I also noticed that the littermate control mice, which are essentially wild-type for Mbp were more lateralized compared to the age-matched pure wild-type cohort from section 3.1 Young wild-type mice appear to be lateralized by 8 weeks of age. As a result, the difference between shiverer and control mice is more pronounced. I conducted a mixed-effect two-way ANOVA with Sidak's multiple comparison test to assess if there was a difference between the laterality of shiverer and control mice at any time-point and found no such difference. This suggested that mice of both groups show a similar time-course of establishment (Figure 9 B).

Based on these data, I concluded that young shiverer mice become comparably lateralized to littermate controls with repetitive testing. This could be due to two reasons. 1) shiverer mice follow the same pattern of establishment like control mice and the lack of compact myelin has no effect in the establishment of behavioural laterality. 2) Repetitive testing on a weekly basis has a learning-like effect on shiverer mice, which makes them appear lateralized comparably to their myelinated controls. Previously data from the lab showed that naïve, adult shiverer mice tested only once, were less lateralized compared to controls (data not shown). Therefore, I wondered if being tested repetitively increased the chances 
of shiverer mice performing as successfully as control mice, leading to their increased laterality.

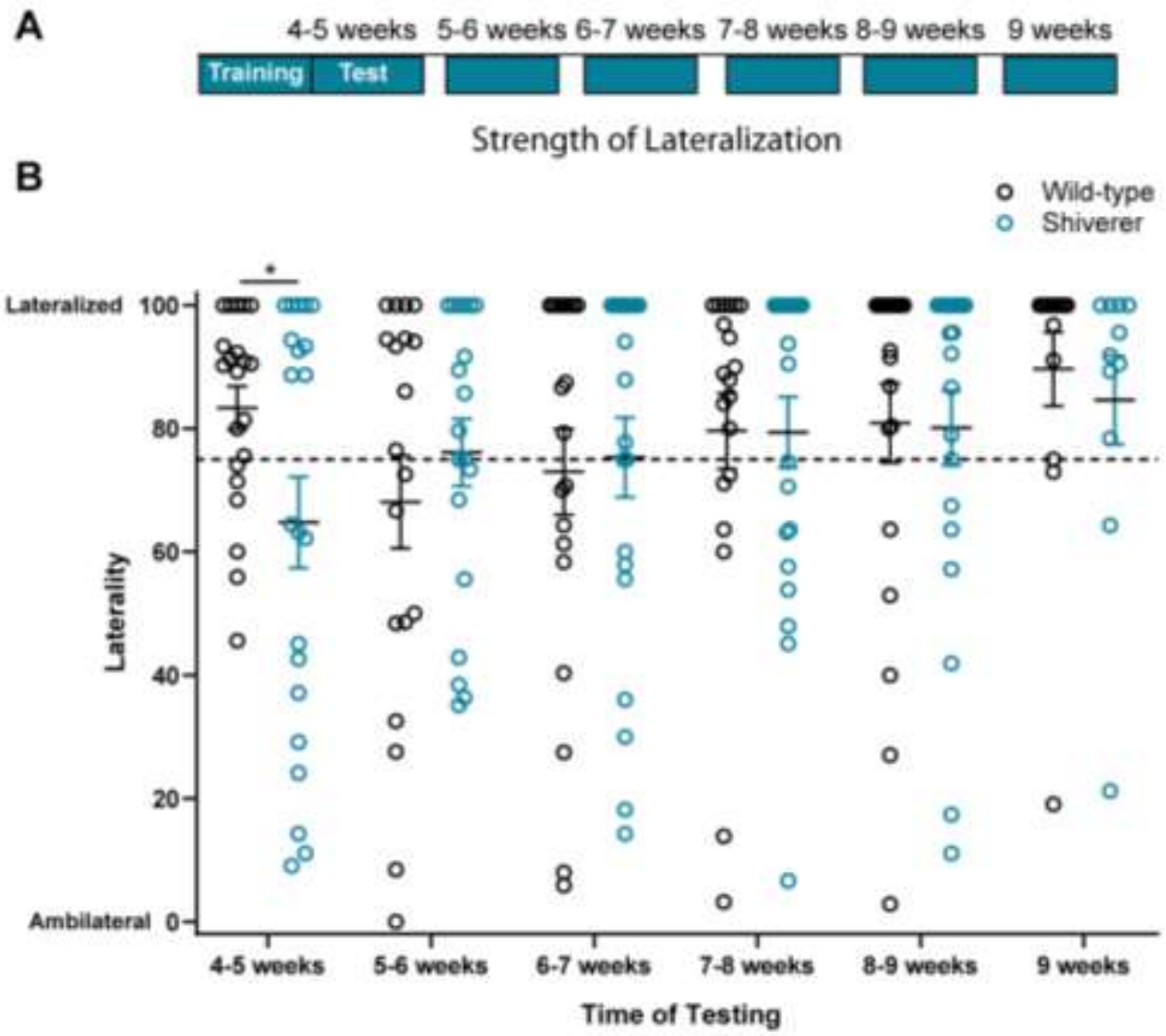

\section{Strength of Lateralization at 4-5 weeks}

D Strength of Lateralization at 9 weeks
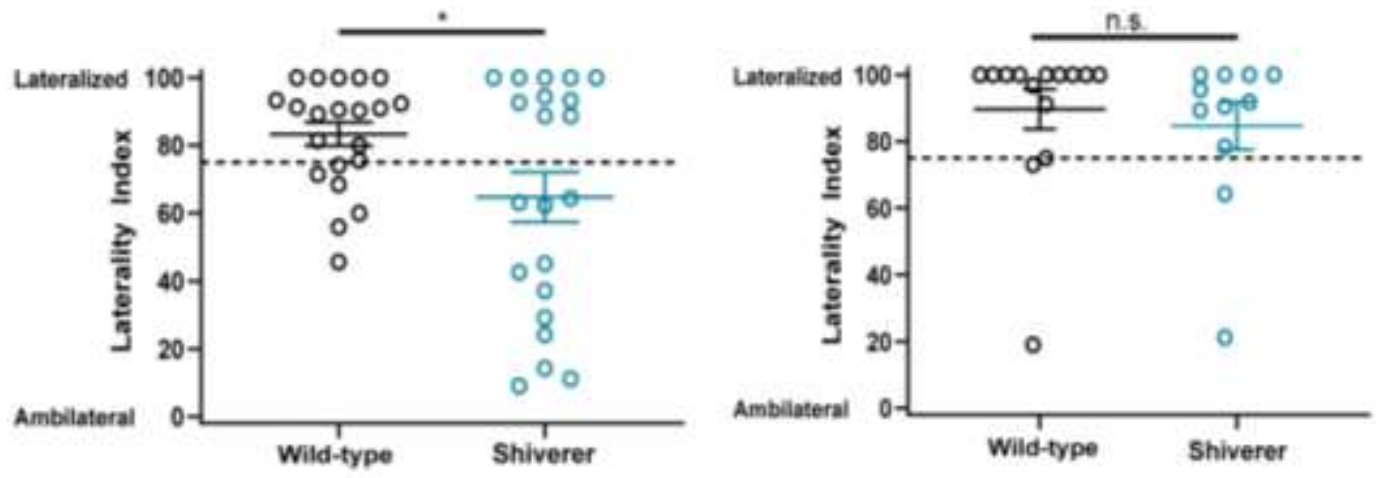
Figure 9: Young shiverer mice become lateralized by 9 weeks of age when tested repetitively.

A) Both female and male shiverer mice and their littermate controls were tested weekly between 4-5 and 9 weeks of age. B) While shiverer mice were less lateralized compared to controls at 4-5 weeks of age, they became lateralized by the 9 week time-point, $n=$ 21 wild-type (black) and $n=21$ shiverer (blue). A mixed-effect two-way ANOVA with Sidak's multiple comparison test to compare the groups at the various time-points, showed no differences between the genotypes at any time-points. C) and D) Individual comparisons between shiverer and control mice at the 4-5 week and 9 week time-points, respectively to show establishment of a comparable laterality in shiverer mice by 9 weeks of age, ${ }^{*}(p$-value $=0.0306)$ and n.s. ( $p$-value $\left.=0.5978\right)$, respectively, two-tailed student's t-test with Welch's correction. Laterality values, $0 \%=$ Ambilateral, $100 \%=$ Lateralized. Mean \pm S.E.M. (error bars).

\subsection{Weekly tested young mice lacking compact myelin show instability with respect to directionality}

When I compared the relative frequency distribution at 4-5 weeks and 9 weeks of age, it was apparent from the U-shaped curve the distribution showed, that the shiverer mice become more lateralized by 9 weeks of age (Figure 10 A). However, when I looked at the directionality of all mice at every test time-point, I noticed that some of the mice tended to switch the direction of their handedness between tests and that this tendency was higher in shiverer mice than control (Figure 10 B). This meant that at a given time-point, mice tended to show an inconsistent directionality despite being strongly lateralized at that time-point. To assess the extent of instability, I calculated an instability index at the 8-week time-point (Equation 6: Instability Index). To compare just how much the directionality at the 8-week timepoint had changed for a given mouse, I plotted the instability index with the given directionality at the 8-week time-point (Figure $10 \mathrm{C}$ ). Since most mice showed an instability index of at most 0.2 , I set an arbitrary threshold at this value. Any mouse showing an instability index above 0.2 was considered unstable. Nearly $50 \%$ of the shiverermice, tested, were unstable with respect to their handedness, whereas only $20 \%$ of control showed instability (Figure $10 \mathrm{D}$ ).

Hence, I concluded that even though shiverer mice appear to be strongly lateralized with repetitive training, they also show an inconsistency in their handedness between tests.

The fact that young shiverer mice, trained repetitively, exhibit a strong preference for paw usage albeit having an unstable handedness begs the question why. Is it 


\section{Results}

due to the training effect alone, observed earlier? Does the absence of compact myelin further increase the likelihood of observing such behaviour? Not to mention one cannot discount the fact that developmental plasticity is ongoing at a very high rate at the same time when I tested these mice. To what extent do each of these factors play a role in defining the establishment of behavioural laterality?

A

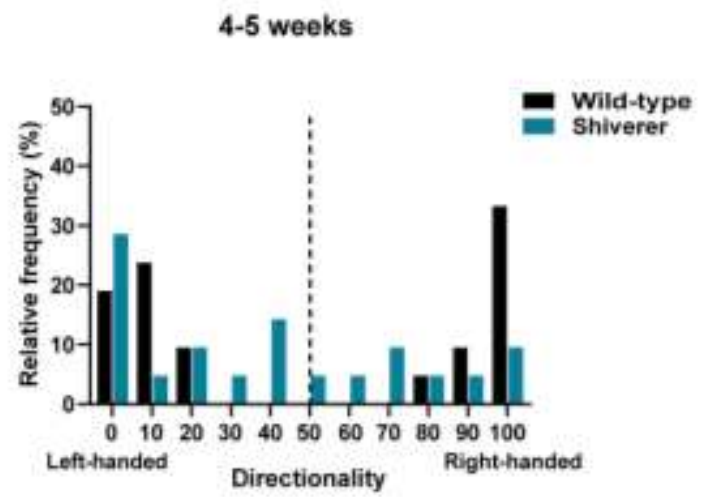

B

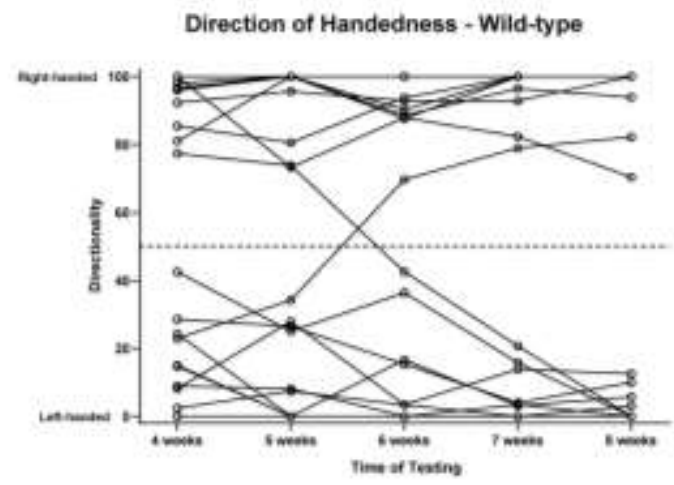

C Correlation between Instability and Handedness

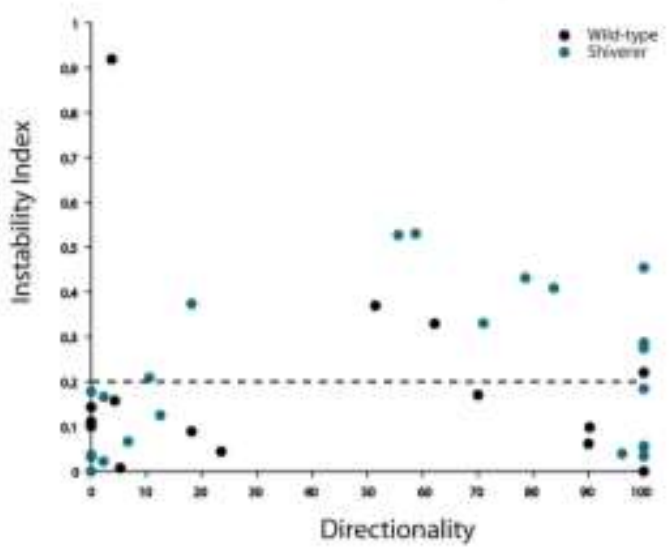

9 weeks

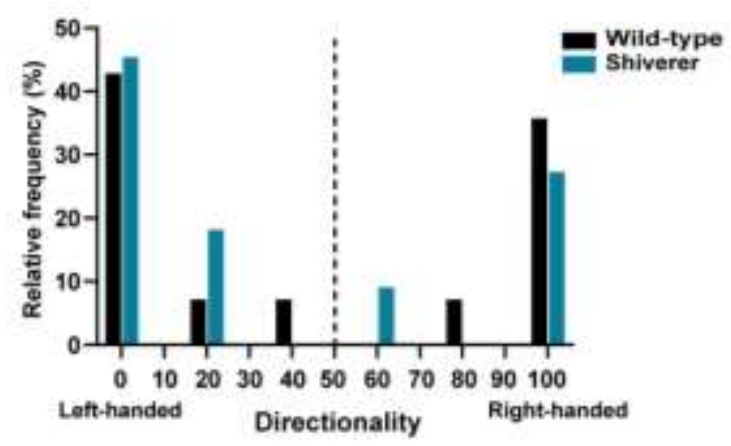

Direction of Handedness - Shiverer

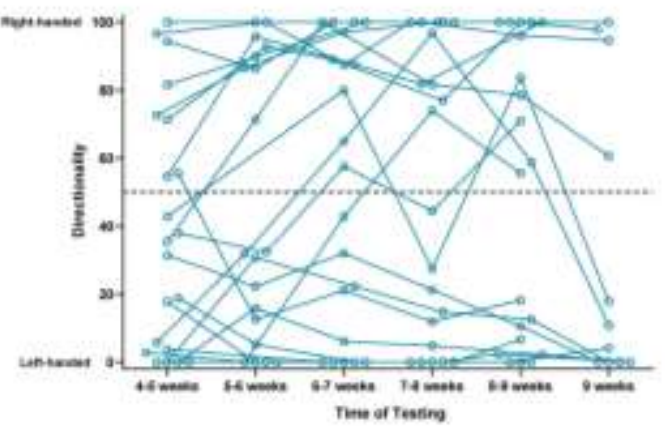

D Instability of Handedness

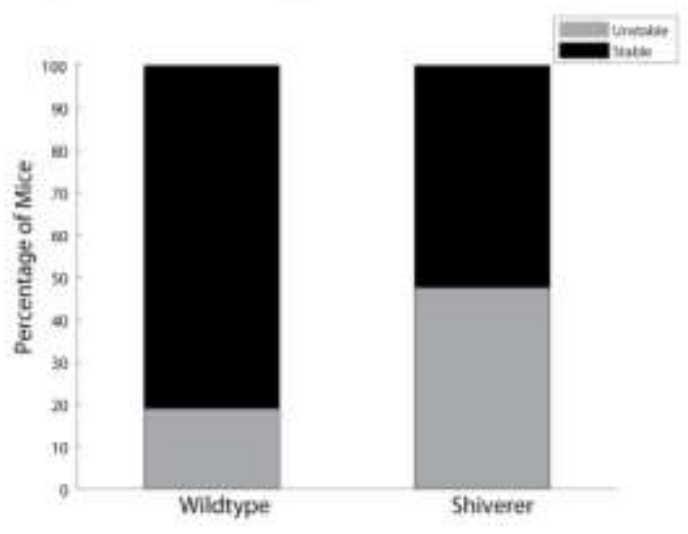


Figure 10: Repetitively tested shiverer mice tend to show more instability with respect to their directionality between tests.

A) Relative frequency distribution of handedness at the 4-5 and 9 week time-points shows that more shiverer mice are ambilateral at the $4-5$ week time-point $\left(x^{2}(9)=11.2381\right)$ and that at the 9-week time-point, they appear to show the U-shaped distribution similar to that exhibited by control mice $\left(x^{2}(9)=7.8439\right)$, with a slight tendency to be more lefthanded. B) Directionality exhibited by all control and shiverer mice at all test time-points, $\mathrm{n}=21$ wild type (black) and $\mathrm{n}=21$ shiverer (blue). C) Correlation between the instability index and the directionality at the 8-week time-point showed that mice unstable in their directionality at the 8-week time-point were also more ambilateral at this time-point. D) A higher proportion of shiverer mice tended to be unstable with respect to their directionality compared to controls. Instability index values, $0<$ instability index $\leq 0.2=$ stable, $0.2<$ instability index $\leq 1=$ unstable. Directionality values, $0 \%=$ Left-handed, $50 \%=$ Ambilateral, $100 \%=$ right-handed. Bin size $=10$.

\subsection{Shiverer mice that are not tested repetitively during development are still lateralized by 8 weeks of age}

To identify whether there is a concomitant effect of absent myelin and repetitive training resulting in an increased laterality or if developmental plasticity also plays a role in the learning of the paradigm, I modified the testing protocol slightly. I began with testing shiverer mice and littermate controls at 4 weeks of age and only tested them next at the 8-week time-point. To assess further the potential effects of repetitive training at a more adult time-point, I tested them once again at 9 and 10 weeks of age (Figure 11 A). I will refer to this cohort as "spaced". While 4week-old shiverer mice are less lateralized than control, they became comparably lateralized by 10 weeks of age (Figure 11 B). A mixed effect two-way ANOVA with Sidak's multiple comparison test revealed that while there is a significant difference between the genotypes at the 4-week time-point ( $p$-value $=0.0237$ ), there is no difference at other test time-points. This suggests that there is only a difference between behavioural lateralization of shiverer and control mice at 4 weeks of age. This held true when I compared the laterality of shiverer and control mice at 4 and 8 weeks of age, respectively. Shiverer mice are less lateralized compared to control at 4 weeks of age ( $p$-value $=0.0042$, Figure $11 \mathrm{C}$ ). However, I observed no significant difference between shiverer and control mice at 8 weeks of age ( $p$ value $=0.1211$, Figure $11 \mathrm{D}$ ) .

The relative frequency distribution at 4 and 8 weeks of age respectively showed that while both shiverer and control mice were ambilateral at 4 weeks of age, the 
control mice showed a typical U-shaped distribution at 8 weeks and the shiverer mice remained more ambilateral (Figure 12 A). When I looked at each individual mouse's performance through all time-points, I found a number of interesting observation. First, some wild type mice that appeared to be right-handed at 4 weeks of age, switched their handedness to the left at 8 weeks (Figure 12 B). Second, most of the unstable switches in handedness were between 4 and 8 weeks of age. There were fewer switches at the 9- or 10-week time-points. I also observed this when I plotted the instability index at 8 weeks of age with the directionality at the time-point. Some wild type mice that appeared to be righthanded were identified as left-handed at 8 weeks (Figure $12 \mathrm{C}$ ). But when I compared the overall percentage of unstable mice for both shiverer and wild-type mice, I found that both groups showed mild instability, especially compared to the weekly tested cohort (Figure 12 D).

With these data, I concluded that it is difficult to comment on the role of myelin in the establishment of laterality. Repetitive testing results in mice learning the paradigm in a reinforced manner, thereby clouding any conclusion that can be drawn regarding their paw lateralization. In addition, the instability in the exhibited handedness further complicates the inference of the results. Since the spaced cohort showed this instability between 4 and 8 weeks and not so much at later time-points, I concluded that developmental plasticity must play a role in the observed instability.

Since shiverer mice lack compact myelin in the entire CNS, it is difficult to pinpoint if the results that I observe are in part due to the dysmyelination of the CC. For this measure, I generated a mouse model lacking compact myelin in a forebrain specific manner (further elaborated in section 3.8 Generation of the cortical dysmyelination mouse models). The results of behavioural lateralization conducted on these mice is shown in 3.11.5 Mice lacking compact myelin the in forebrain show no behavioural lateralization impairment. 
A

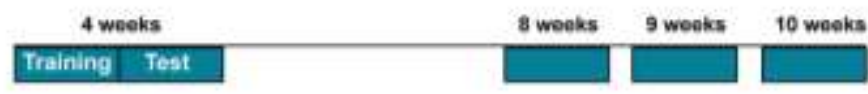

B

Strength of Lateralization

- Wild-type

- Shiverer

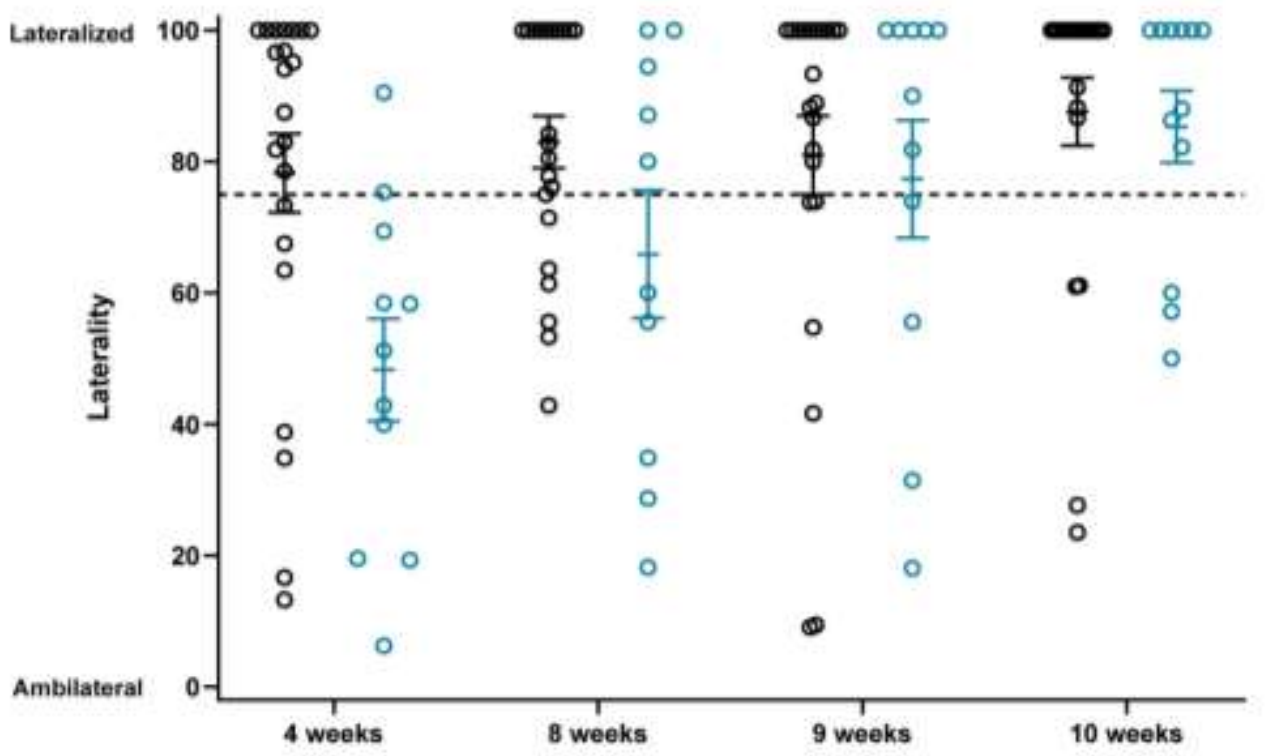

C Strength of Lateralization at 4 weeks

D Strength of Lateralization at 8 weeks
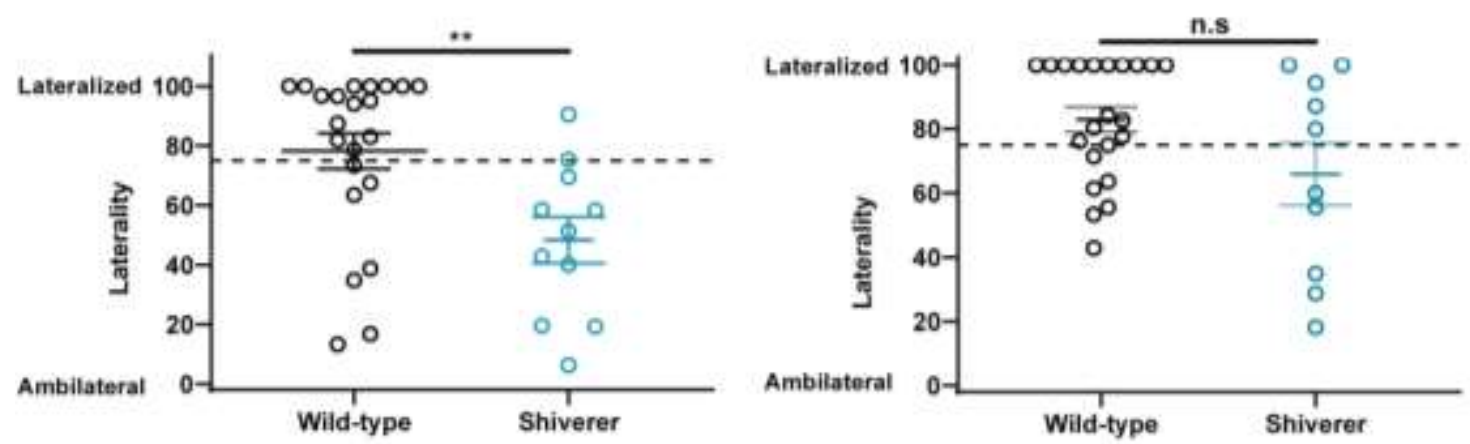

Figure 11: Shiverer mice not tested repetitively during development are still lateralized by 8 weeks of age.

A) Both female and male shiverer mice and their littermate controls were tested first at 4 weeks of age and then again repetitively starting at 8 weeks of age, until 10 weeks of age. B) While shiverer mice appear less lateralized compared to controls at 4 weeks of age, they are comparably lateralized by the 8 week time-point, $n=22$ wild type (black) and $n$ $=12$ shiverer (blue). A mixed effect two-way ANOVA with Sidak's multiple comparison test to compare the groups at the various time-points, showed a significant interaction between the genotype and time components, ${ }^{*}(p$-value $=0.0371) . C$ ) and $\left.\mathbf{D}\right)$ Individual comparisons between shiverer and control mice at the 4 week and 8 week time-points, respectively to show establishment of a comparable laterality in shiverer mice by the 8 week time-point, ${ }^{* *}(p$-value $=0.0042)$ and n.s. $(p$-value $=0.1211)$, respectively, MannWhitney test. Laterality values, $0 \%=$ Ambilateral, $100 \%=$ Lateralized. Mean \pm S.E.M. (error bars). 
A
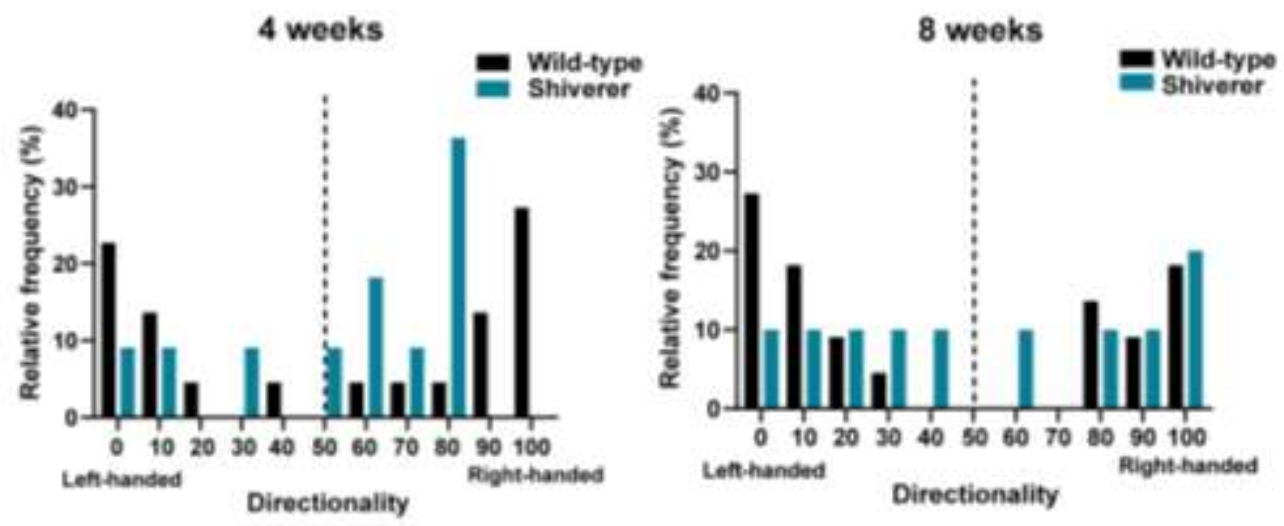

B Direction of Handedness - Wild-type

Direction of Handedness - Shiverer
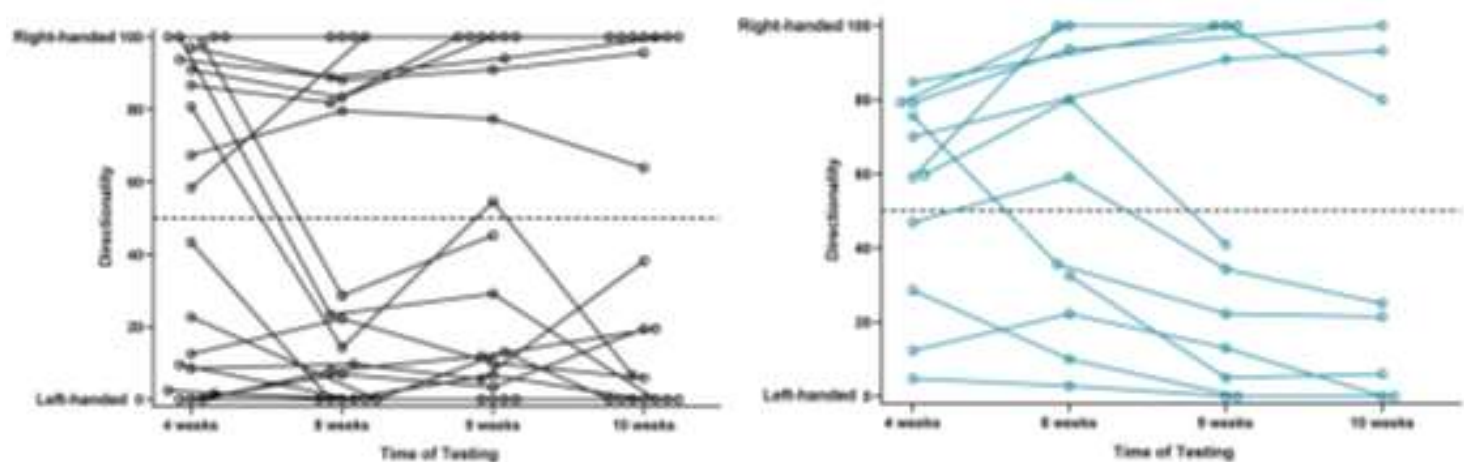

C Correlation between Instability and Handedness

D

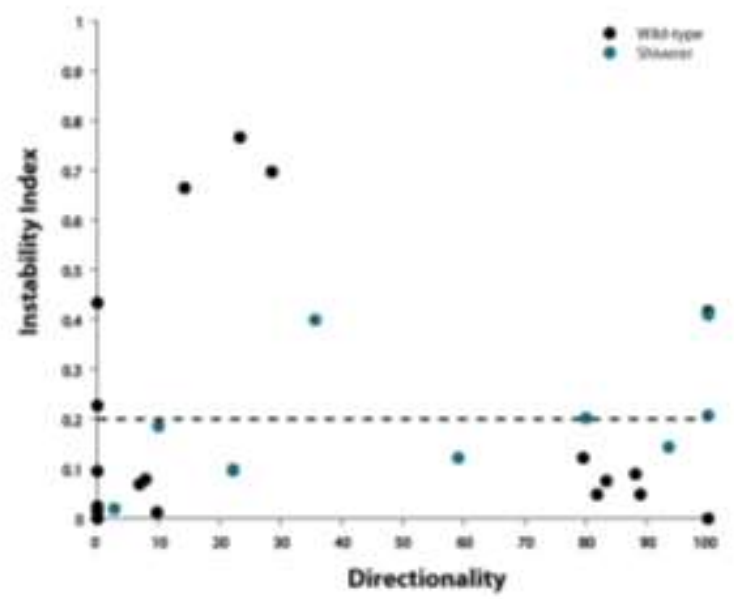

Instability of Handedness
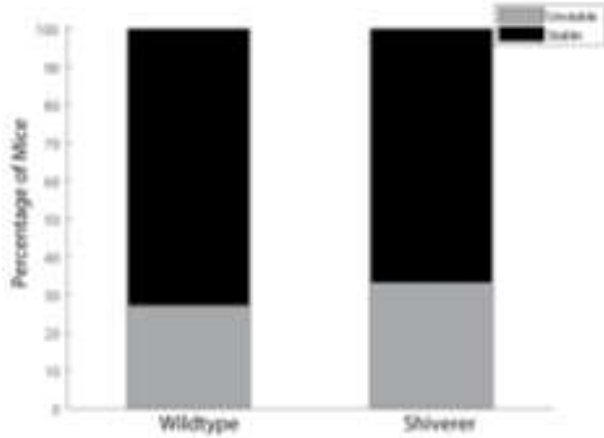
Figure 12: Young shiverer mice not tested repetitively are more stable in the handedness they exhibit.

A) Relative frequency distribution of shiverer and control mice at 4 and 8 weeks of age. The distribution curve of shiverer mice do not show the typical U-shaped distribution like the control mice, suggesting that shiverer mice are more ambilateral at 8 weeks of age. B) Direction of handedness of all mice tested at 4, 8, 9 and 10 weeks of age shows that both shiverer and control mice are more likely to show inconsistency in handedness before 8 weeks of age, $n=22$ wild type (black) and $n=12$ shiverer (blue). C) Correlation between instability index and handedness at 8 weeks of age suggests that the control mice were more unstable, in that, they became left-handed at the 8-week time-point despite being right-handed at the 4-week time-point. D) Overall, the same proportion of shiverer and control mice are unstable. Instability index values, $0<$ instability index $\leq 0.2=$ stable, $0.2<$ instability index $\leq 1=$ unstable. Directionality values, $0 \%=$ Left-handed, $50 \%=$ Ambilateral, $100 \%=$ right-handed. Bin size $=10$.

\subsection{Generation of the cortical dysmyelination mouse models}

Myelination is a process that begins right before birth and continues well into adulthood, in humans. Formation of new myelin in adulthood is called adaptive myelination and is believed to occur in response to neuronal activity (Choi et al., 2019; Gyllensten and Malmfors, 1963). Adaptive myelination is said to mediate learning of tasks such as juggling and learning a new musical instruments in adulthood (Bengtsson et al., 2005; Scholz et al., 2009). To investigate the role of cortical myelination in higher-brain functions, I used two mouse lines in which Mbp is knocked out, in a conditional manner, only in the forebrain. This renders the forebrain, comprising the cerebrum, hypothalamus and thalamus, devoid of compact myelin. To do so, I crossed the MBPflox mouse line with either Emx1IRES-Cre or Foxg1-IRES-Cre. The MBPflox line, generated by Dr. Wiebke Möbius of the Max Planck Institute for Experimental Medicine, contains loxP sites flanking Exon 1 of the classical Mbp locus (Meschkat et al., 2020).

The first Cre line I crossed with the MBP flox mice, was Emx1-IRES-Cre mouse line (Gorski et al., 2002). Emx1 codes for homeodomain protein and is expressed by excitatory neurons and glia in the cortex (Gorski et al., 2002). Emx1 is expressed, in the mouse embryo, at E12.5 (Simeone et al., 1992). Gorski and colleagues, inserted an internal ribosome entry site and a Cre recombinase coding region along the Emx1 gene (Gorski et al., 2002). When crossed with Mbp $p^{f / f l}$ mice, the resultant progeny either lacked Mbp expression in their forebrain (cKO) or appeared normal like wild type (control) mice. For simplicity, I shall refer to these mice as EMXMBP. The second Cre line was the Foxg1-IRES-Cre mouse line 
(Kawaguchi et al., 2016). Foxg1 or the forkhead box protein G1 is a telencephalonspecific gene crucial for determining the number of cells in the cortex. Although how it mediates this process is unknown, knocking out Foxg1 results in cerebral hypoplasia (Hanashima et al., 2004). It is expressed on mouse embryonic day E8.5 (Kawaguchi et al., 2016). I will refer to them as FOXMBP from now on.

A representation of the breeding scheme, which resulted in the generation of the two forebrain-specific Mbp knockout mouse models, is shown in Figure 13.

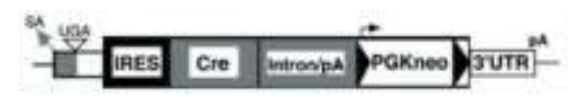

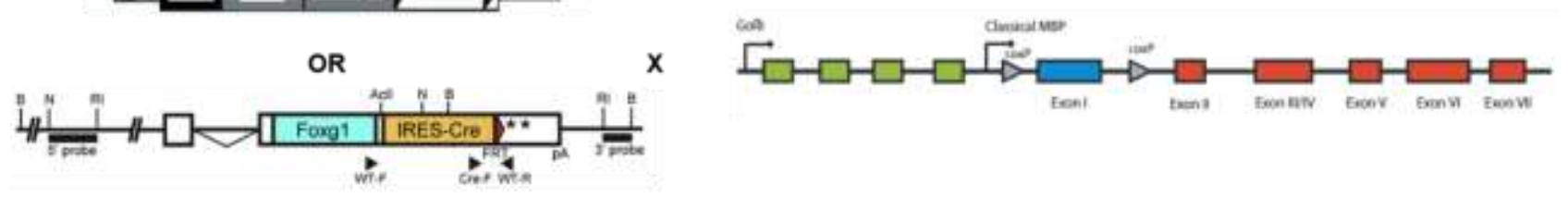

Figure 13: Schematic showing how the two mouse lines for cortical dysmyelination were generated.

Emx1-IRES-Cre or FOXG1-IRES-Cre mice were crossed with MBPFLX mice to generate mice lacking compact myelin in the forebrain. Schematic constructs of Emx1-IRES-Cre taken from Gorski et al., 2010; FOXG1-IRES-Cre taken from Kawaguchi et al., 2016; MBPFLX taken from Meschkat et al,. 2020.

\subsection{Characterization of the EMXMBP mice}

\subsubsection{EMXMBP mice show a reduction in MBP in the forebrain}

To validate the successful knockout of $\mathrm{Mbp}$ in the forebrain, I isolated tissue from the WM, cortex and cerebellum of 6-month-old male mice. I prepared lysates of the regions, mentioned above, and estimated MBP content in these regions. I normalised the MBP intensity to the total protein content visualised with Fast Green staining. I then plotted the relative MBP intensity normalised to the control MBP intensity. Two bands at $18 \mathrm{kDa}$ and another at $14 \mathrm{kDa}$ were observed when the blots were stained for MBP. For the WM lysate, MBP levels, in case of cKO mice, was comparable to control mice ( $p$-value $=0.2495$, Figure $14 \mathbf{A}$ ). In case of the 


\section{Results}

cortex, there was a significant reduction in MBP in the $\mathrm{cKO}$ compared to control mice $(p-$ value $=0.0452$, Figure 14 B). Since Emx1-IRES-Cre does not recombine in the cerebellum, the cerebellum of cKO mice had comparable levels of MBP to control mice ( $p$-value $=0.0760$, Figure $14 \mathrm{C}$ ). Since the inter-sample variability was so high, the reduction in MBP levels in the different regions was mild. This can be attributed to human error in sample collection and preparation prior to the western blot protocol.

To further validate the reduction in MBP in the EMXMBP mice, I performed immunohistochemistry on $5 \mu \mathrm{m}$ thick, sagittal, paraffin-embedded sections of 4month-old male mice. As predicted, the MBP levels were reduced in the forebrain of cKO mice compared to controls, whereas areas such as the cerebellum were unaffected and appeared normally myelinated like that of control mice (Figure 15 A). When I quantified the percentage of area positive for MBP in the $\mathrm{CC}, \mathrm{I}$ found a 4-fold reduction in MBP in the $\mathrm{cKO}$ mice compared to control mice ( $\mathrm{p}$-value = 0.0003 , Figure $15 \mathrm{~B}$ ). On quantifying the percentage of area positive for MBP in the cortex, I found a remarkable 14-fold reduction in MBP in the cKO mice compared to control mice ( $p<0.0001$, Figure $15 \mathrm{C}$ ).

According to these data, I confirmed that the recombination in the mice was successful and that a forebrain-specific dysmyelination phenotype could be observed, exemplified by the stark reduction in MBP in forebrain structures such as the CC and cortex. Meanwhile, structures outside the forebrain such as the cerebellum were normally myelinated.

Next, I decided to investigate the status of other cell-types in this mouse model. This was of particular interest to me because shiverer mice do not live past 3 months of age, and hence I was curious to know if there is any exacerbated gliosis when a shiverer-like system is allowed to persist in the manner we have achieved. 
A

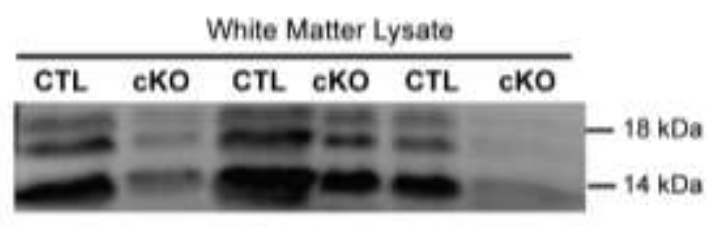

B

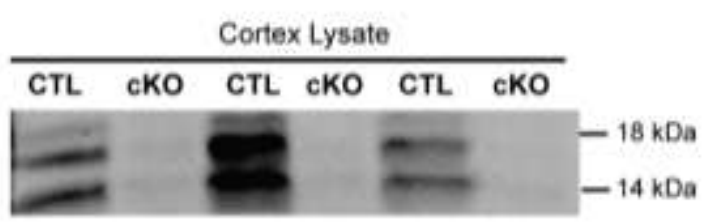

C

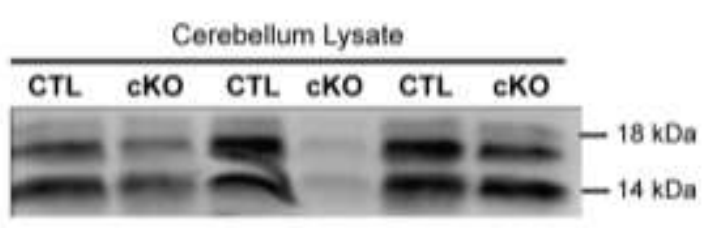

White Matter
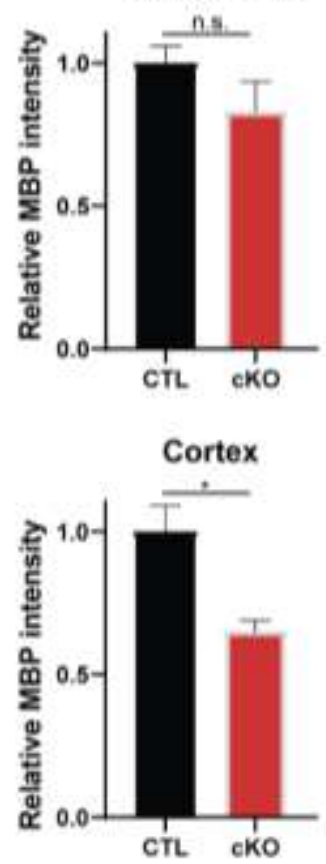

Cerebellum

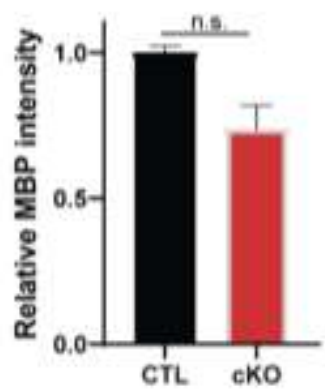

Figure 14: Protein estimation of MBP in EMXMBP shows a reduction in protein levels in the forebrain.

A) MBP levels in the white matter lysate of male cKO EMXMBP (red) mice are reduced but not significantly less compared to controls (black), n.s. ( $p$-value $=$ 0.2495). B) MBP levels in the cortex of cKO EMXMBP mice are significantly reduced compared to control, * $(p$-value $=0.0452)$. C) MBP levels in the cerebellum of cKO EMXMBP mice remain the same compared to control, n.s. (p-value = 0.0706). T-test with Welch's correction, $n=3$ for both groups. Mean \pm S.E.M. 


\section{Results}

A

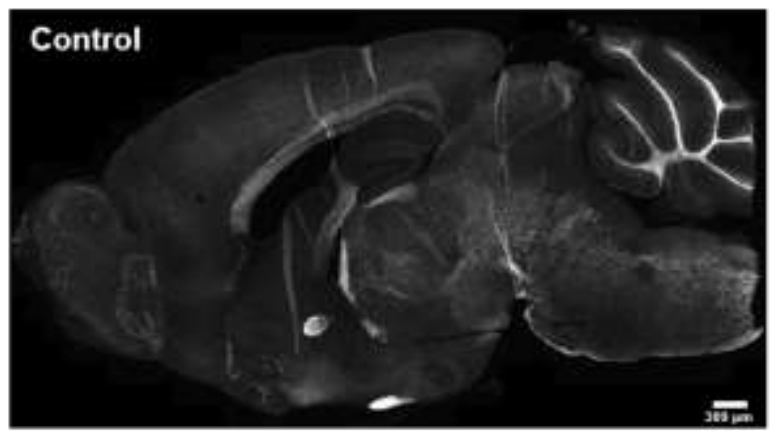

B

\section{Corpus Callosum}

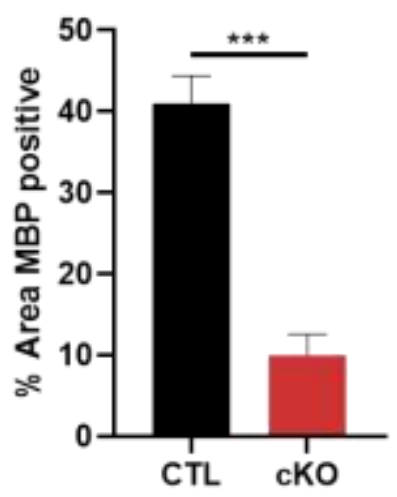

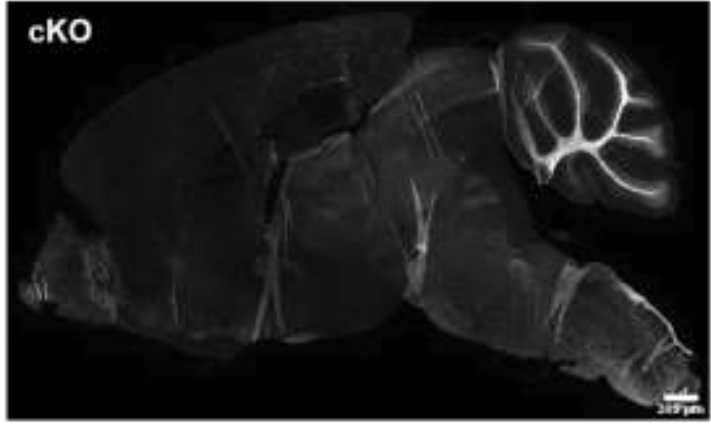

C

Cortex

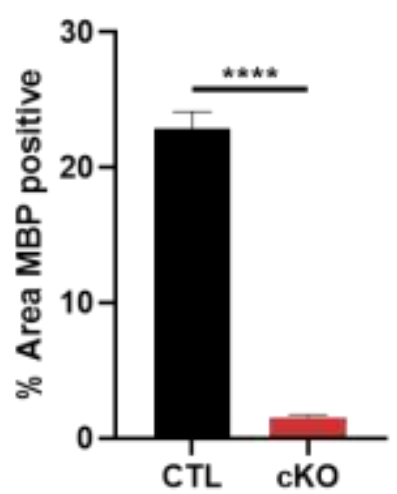

Figure 15: Immunohistochemical quantification of MBP shows a reduction in EMXMBP mice.

A) representative images of sigittal sections $(5 \mu \mathrm{m})$ of 4 -month-old male control and cKO mice stained for MBP shows a remarkable reduction in MBP only in the cKO forebrain. Other structures are well preserved with respect to MBP expression. B) Quantification of the percentage of area positive for MBP in the CC shows a significant reduction of MBP in the cKO mice (red) comapred to control mice (black), ${ }^{* * *}(p$-value $=0.0003)$. C) Quantification of percentage of area positive for MBP in the cortex shows a significant reduction in MBP in the cKO mice compared to control mice, ${ }^{* * * *}(p<0.0001)$. Unpaired two-tailed student's t-test, $n=4$ for both groups, Mean \pm S.E.M., scale bar $309 \mu \mathrm{m}$.

\subsubsection{EMXMBP mice show a mild gliosis phenotype in the corpus callosum}

Despite being discovered nearly 50 years ago, very little is known about the behaviour of other glial cell-types in the absence of compact myelin in the shiverer mouse model. There has been a reported increase in the number of lba1 positive microglia in the shiverer spinal cord, however, no inflammatory response is observed on the transcriptomic level. (Nomura et al., 2013). To this effect, it was necessary to investigate the microenvironment, especially of heavily myelinated regions such as the $\mathrm{CC}$, in the absence of MBP. Paraffin-embedded sections were 


\section{Results}

stained with classic markers for neuropathology. The $C C$ and cortex of 4-monthold cKO mice and control EMXMBP were quantified for the following: 1) glial fibrillary acidic protein (GFAP), 2) Iba1, 3) Mac3, 4) CD3, and amyloid precursor protein (APP) (Figure 16 F). A slight but significant increase in GFAP, a marker for astrogliosis, was observed in the EMXMBP cKO CC compared to controls ( $p$ value $=0.0147)$. However, they were unchanged in the $c K O$ cortex ( $p$-value $=$ 0.8163 ), suggesting no astrogliosis occurs in the cortex (Figure $16 \mathrm{~A}$ ). The $\mathrm{CC}$ of cKO mice also showed a marginal increase in lba1 positive staining compared to control ( $p$-value $=0.0361$ ), which was indicative of more microglia being present. However, in case of the cortex, it was comparable to control mice ( $p$-value $=$ 0.4368 , Figure 16 B). to check whether these excess microglia were activated and hence capable of inducing inflammation, Mac3 positive staining was quantified and was unchanged in both CC ( $p$-value $=0.4)$ and cortex ( $p$-value $=0.4665)$ of the cKO mice compared to controls (Figure 16 C). This means that even though there are excess microglia present in the CC of EMXMBP cKO mice, they do not necessarily cause inflammation. CD3 is a marker for T-lymphocytes and their presence is indicative of a compromised blood-brain barrier. Number of CD3 positive cells was slightly increased in the $C C$ ( $p$-value $=0.0323$ ) but not in the cortex ( $p$-value $=0.7716$ ) of EMXMBP cKO mice (Figure $16 \mathrm{D}$ ). APP swellings are often indicative of axonal degeneration. To assess the health of axons in the CC and the cortex, number of APP swellings were quantified (Figure $16 \mathrm{E}$ ). No significant increase in APP swellings were found in both the cKO CC ( $p$-value = 0.9702 ) and cortex ( $p$-value $=0.3606$ ). This means that despite lacking compact myelin, the axons in the CC and cortex of EMXMBP mice are healthy.

Moreover, I noticed that a large number of haematoxylin stained nuclei were present in the cKO CC and cortex. The mild increase in astrocytes and microglia alone could not account for this increase. Therefore, I postulated that these might be OPCs and/or OLs. 

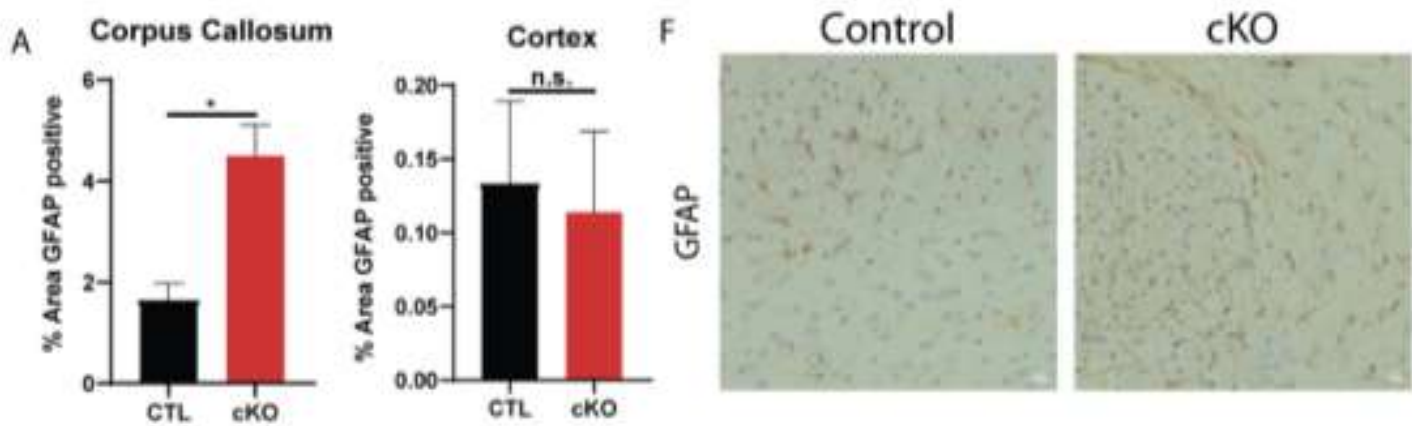

B
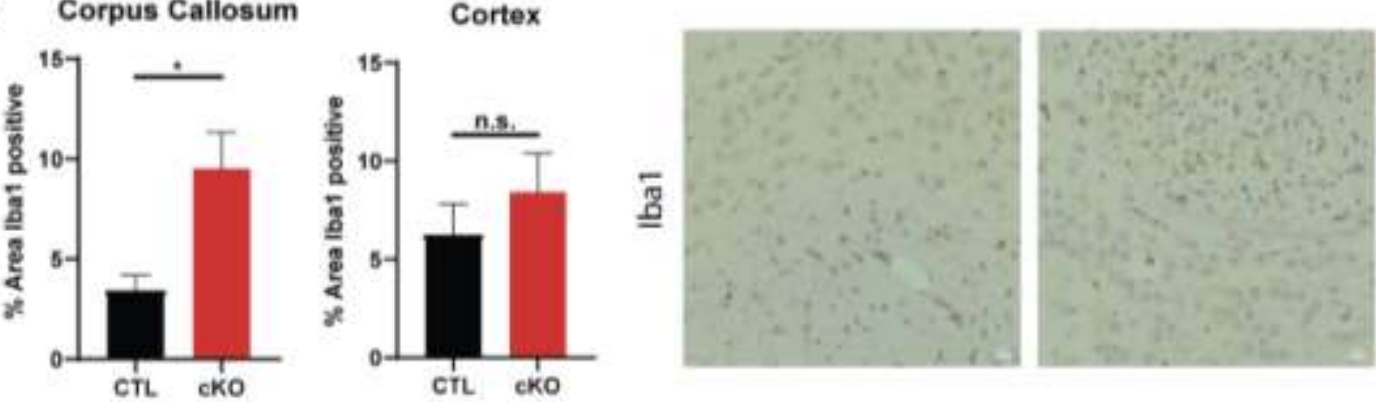

C Corpus Callosum
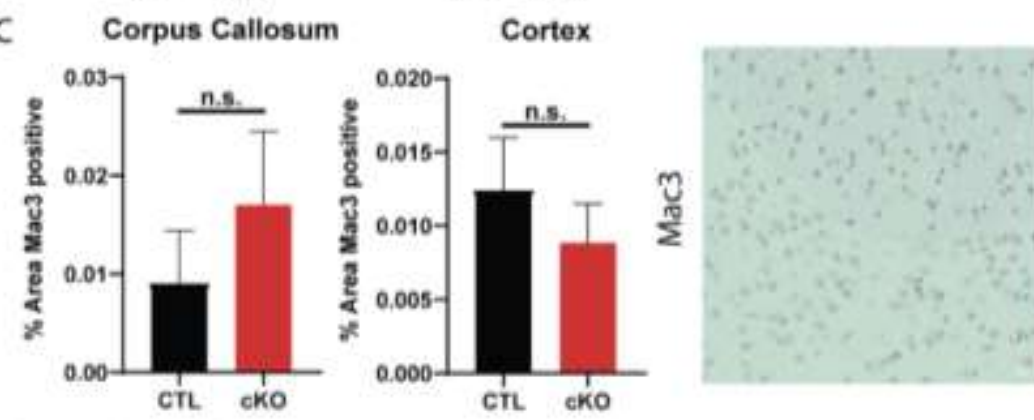

D Corpus Callosum

Cortex
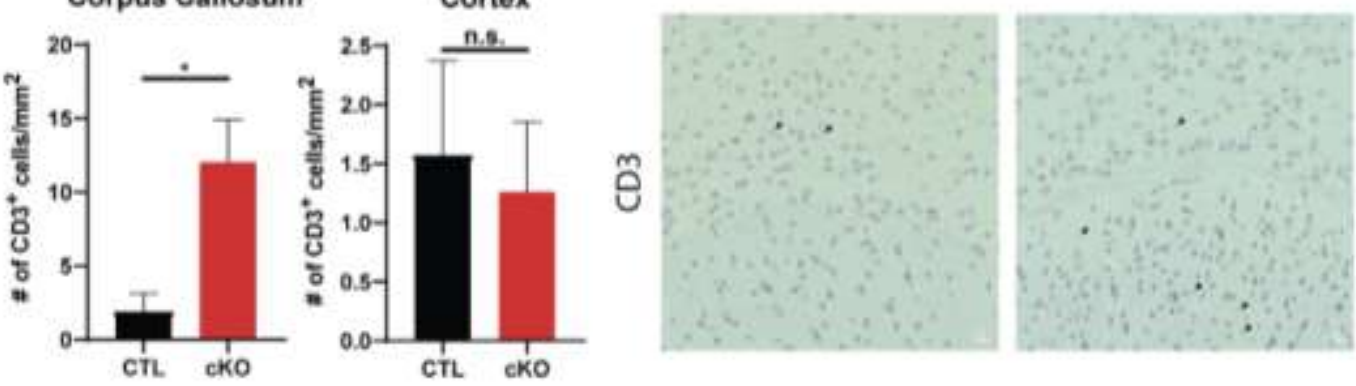

E
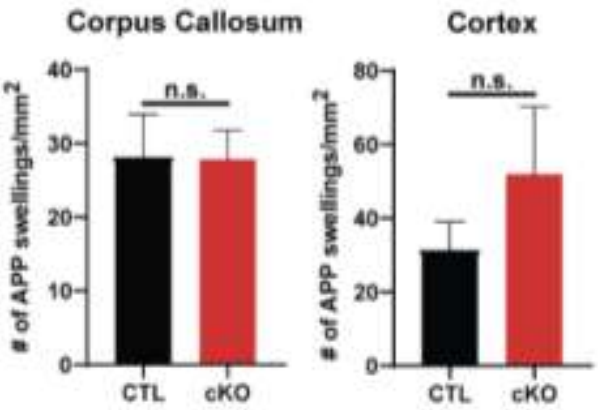

$\frac{\grave{2}}{<}$
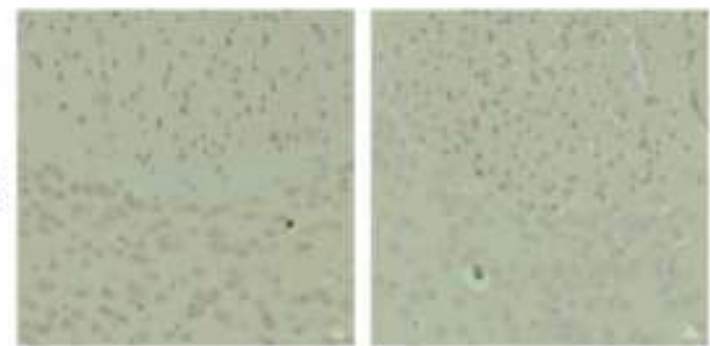
Figure 16: EMXMBP mice show a mild form of gliosis at $\mathbf{4}$ months of age.

A) Percentage of area positive GFAP, a marker for astrogliosisis, was slightly elevated in the cKO CC but comparable in the cortex, ${ }^{*}(p$-value $=0.0147, C C)$, n.s. ( $p$-value $=$ 0.8163, cortex). B) Percentage area positive for lba1, a microglial marker, was increased in cKO CC but not in the cortex, ${ }^{*}(p$-value $=0.0361, C C)$, n.s. $(p$-value $=$ 0.4368, cortex). C) Percentage area positive for Mac3, a microglial activation marker, was not changed in both $\mathrm{CC}$ and cortex of $\mathrm{cKO}$ mice, n.s. (p-value $=0.4, \mathrm{CC} ; 0.4665$, cortex). D) Number of CD3 positive cell density is an indication of T-lymphocytic infiltration. A slight increase in CD3 positive cells was observed in the cKO CC. However it was comparable in the cortex of the cKO mice, ${ }^{*}(p$-value $=0.0323, C C)$, n.s. $(p$-value $=0.7716$, cortex. E) Increased number of APP swellings is indicative of axonal degeneration. This was not observed in both CC and cortex of cKO mice, n.s. (p-value $=0.9702, \mathrm{CC} ; 0.3606$, cortex). F) Respresentative images of the CC and cortex of 4month-old cKO and control EMXMBP mice stained for GFAP, Iba1, Mac3, CD3 (arrowheads indicate CD3 positive cells) and APP (arrowheads indicate APP swellings), respectively. Unpaired two-tailed student's t-test or Mann-Whitney test, $\mathrm{n}=3$ for both groups Mean \pm S.E.M., scale bar $5 \mu \mathrm{m}$. Data acquired and analysed by Jonas Hemesath, Master's student.

\subsubsection{EMXMBP mice seem to have more OPCs and OLs in the corpus callosum}

So far, studies have focused on transplantation of healthy oligodendroglial lineage cells into shiverer brains both in vitro (Bin et al., 2012) and in vivo (Gumpel et al., 1983; Yandava et al., 1999). However, little is known of the health of the resident oligodendrocytes. What happens to them when they are incapable of forming compact myelin? Do they undergo apoptosis or remain there attempting to unsuccessfully myelinate fibres? When I saw more nuclei than the GFAP and Iba1 staining could account for, I decided to investigate if these excess cells were OPCs or OLs. Olig2 is a marker for all oligodendroglia lineage cells, which includes both OPCs and OLs. In addition, I co-stained the paraffin-embedded sections of 6month-old EMXMBP mice with carbonic anhydrase II (CAII) that is specifically expressed by mature, myelinating OLs. I quantified cells that were Olig2-positive and CAll-negative as OPCs and Olig2-positive, CAll-positive cells as OLs, in the CC (Figure 17 A) and cortex (Figure $17 \mathrm{D}$ ) of EMXMBP mice. I found that there was a trend towards an increase in cell density of OLs but not OPCs, in the CC (Figure $17 \mathbf{B}$ and $\mathbf{C}$ ). There was no trend of that sort in the cortex (Figure $17 \mathbf{E}$ and F), however. No statistical analysis could be performed due to the low sample number of the cKO. 


\title{
Results
}

These data suggest that more OPCs are recruited, especially in the WM, in the absence of compact myelin. They rapidly differentiate into OLs, perhaps to ameliorate the dysmyelination phenotype. However, they fail to do so. Moreover, the underpinning mechanism that recruits these OPCs is yet to be elucidated.

A
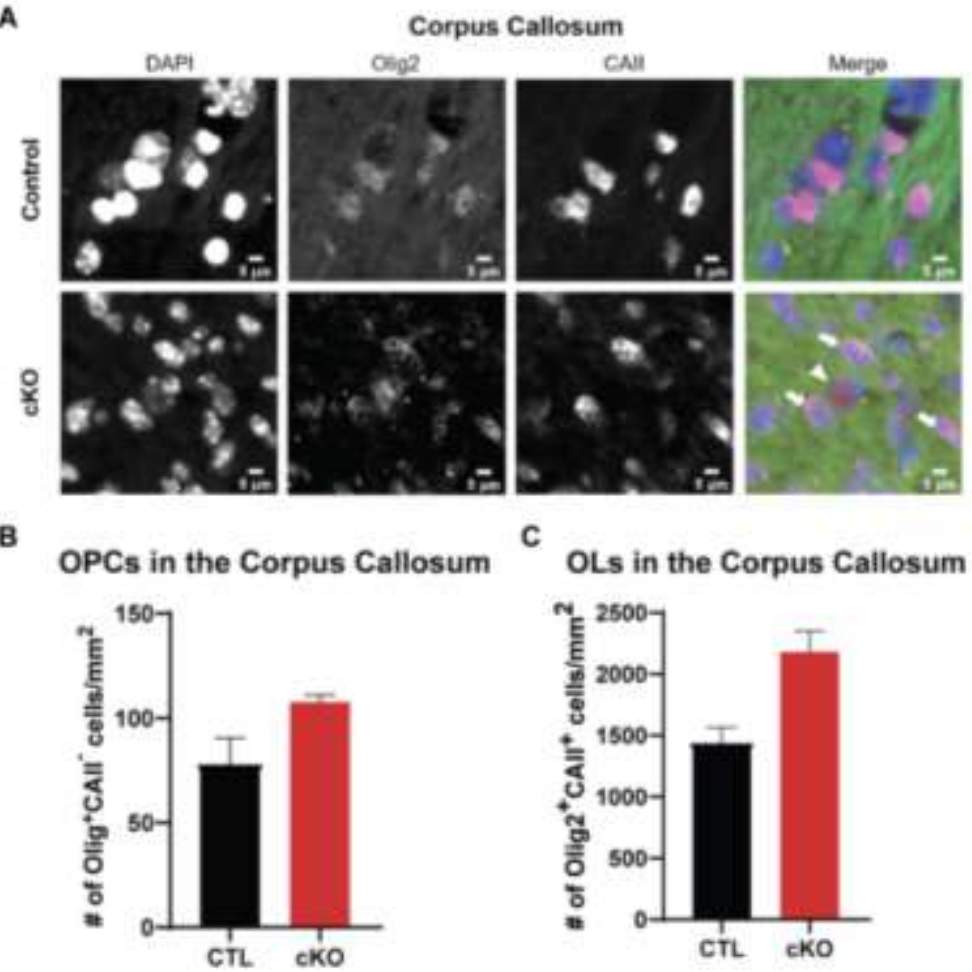

D
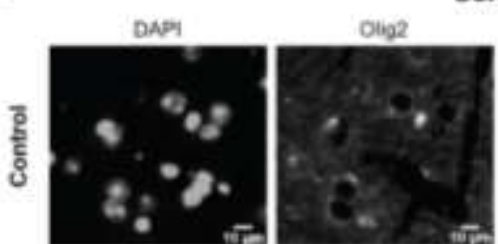

Cortex
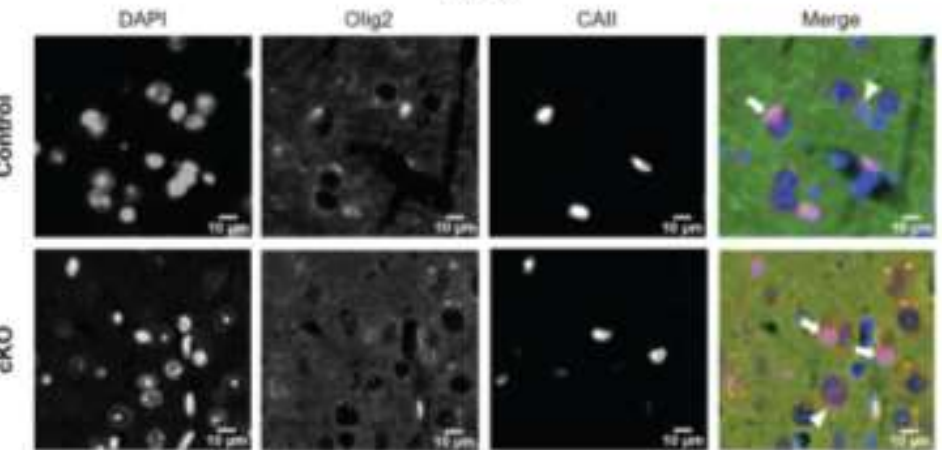

E
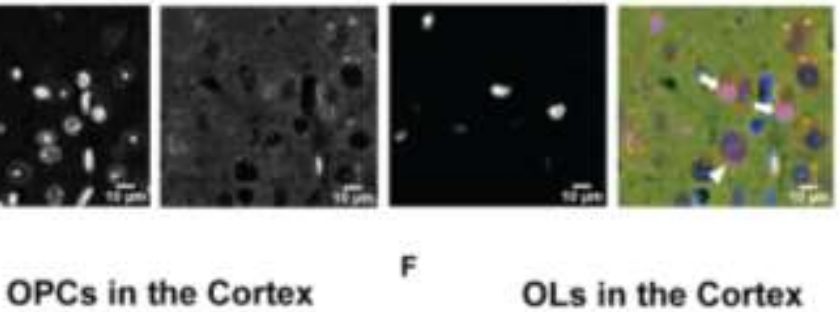

F

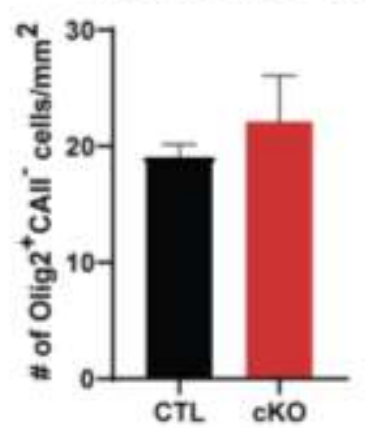

\begin{abstract}
OLs in the Cortex
\end{abstract}

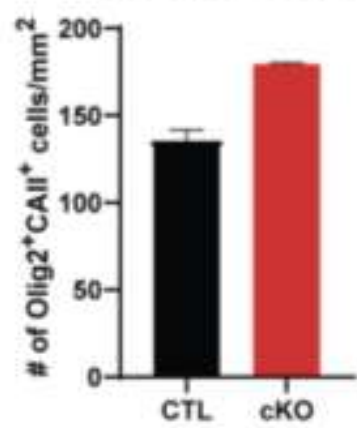


Figure 17: EMXMBP mice appear to have more OLs in the CC and cortex.

A) and D) Representative images of OPCs and OLs in CC and cortex of 6-month-old EMXMBP cKO and control mice. B) and C) There is a slight trend towards an increase in the number of OPCs and OLs in the CC of cKO EMXMBP mice (red) compared to controls (black). E) and F) No increase in the number of OPCs and OLs in the cortex is observed in the cKO EMXMBP mice compared to controls, $n=3$ control and $2 \mathrm{cKO}$, Mean \pm S.E.M., scale bar $5 \mu \mathrm{m}$ (CC), $10 \mu \mathrm{m}$ (cortex).

\subsubsection{Larger calibre axons are preferentially myelinated compared to smaller calibre axons in the rostral corpus callosum of EMXMBP mice}

The ultrastructure of the shiverer CNS has been extensively studied since the 70s (Bird et al., 1978; Dentinger et al., 1982; Inoue et al., 1981; Privat et al., 1979). To assess whether the EMXMBP mice have a "shiverer phenotype", which is characterised by the appearance of non-compact myelin wraps around axons and an increase in unmyelinated axons, in the forebrain, the CC of 4-month-old EMXMBP mice were examined with EM. Compared to control mice, the rCC of EMXMBP cKO mice shows a dorsal-ventral disparity with respect to the dysmyelination phenotype. The dorsal CC is dysmyelinated whereas the ventral CC appears to be normally myelinated, like that of control mice (Figure $18 \mathrm{~A}$ ). The EMXMBP show an overall $14 \%$ increase in the appearance of shiverer axons (non-compact myelin around axons, Figure 18 B) compared to controls. Since EMXMBP mice have both normally myelinated and shiverer axons in their $\mathrm{CC}$, the absolute number of compact and shivereraxons per $180 \mu \mathrm{m}^{2}$ was quantified. While the absolute number of axons with compact myelin remain comparable between control and cKO mice ( $p$-value $=0.35$ ), there is a significant increase in the number of shiverer axons in the EMXMBP cKO mice ( $p$-value $=0.05$, Figure $18 \mathrm{C}$ ). To examine what kind of axons were myelinated in the EMXMBP mice; the axon calibres from randomly sampled EM images were determined and binned into 200 $\mathrm{nm}$ sized bins. The relative frequency distribution of the axon calibres was generated (Figure $18 \mathrm{D}$ ). This allowed one to see not only what percentage of axons were normally myelinated in both control and cKO mice, but also how many axons were shiverer-like axons (in the cKO mice). A calibre-shift in the cKO mice is observed with respect to normally myelinated axons compared to control mice 


\section{Results}

$(p<1 e-10)$. This means, the large-calibre axons were normally myelinated in EMXMBP mice. In addition, small-calibre axons in cKO mice were more likely to be shiverer-like in appearance than in controls ( $p$-value $=0.0003)$. Moreover, there were significantly more shiverer-axons than compact axons in the cKO mice $(p<1 e-10)$. G-ratio analysis of normally myelinated axons in cKO and control mice showed that while the g-ratio of large-calibre axons was comparable in both groups, the small-calibre axons in the cKO mice tended to be hypomyelinated (Figure $18 \mathrm{E}$ ). 


\section{Results}
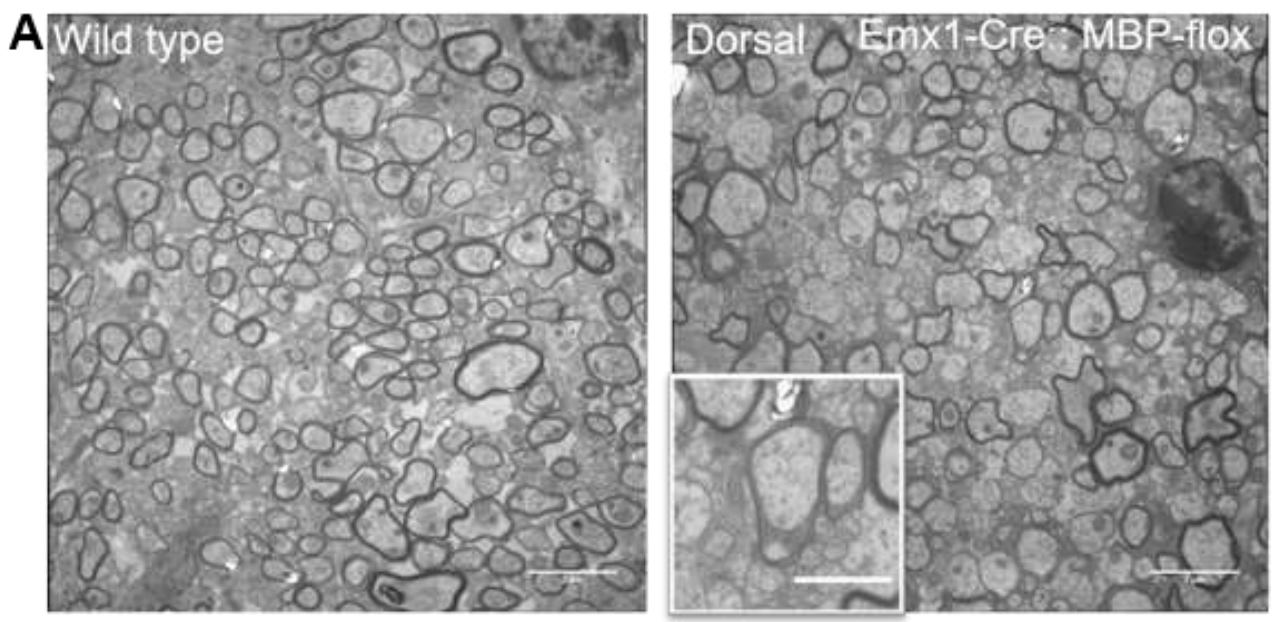

B
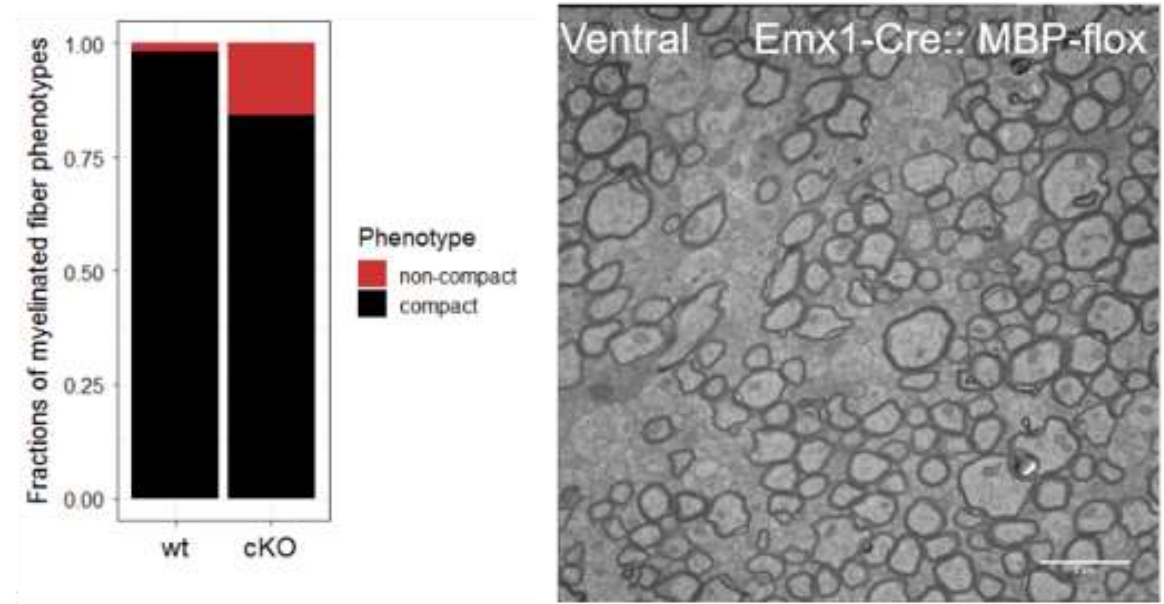

C

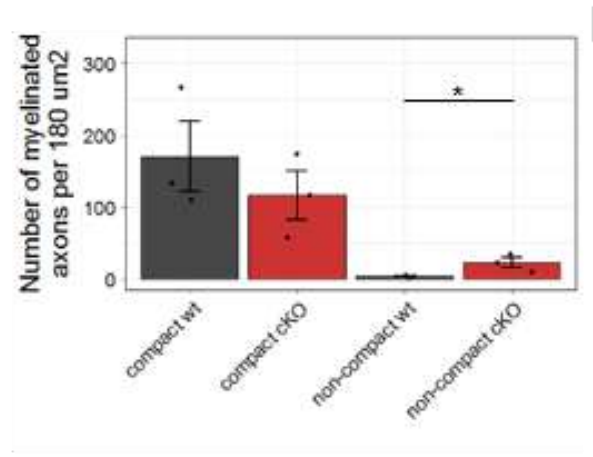

D
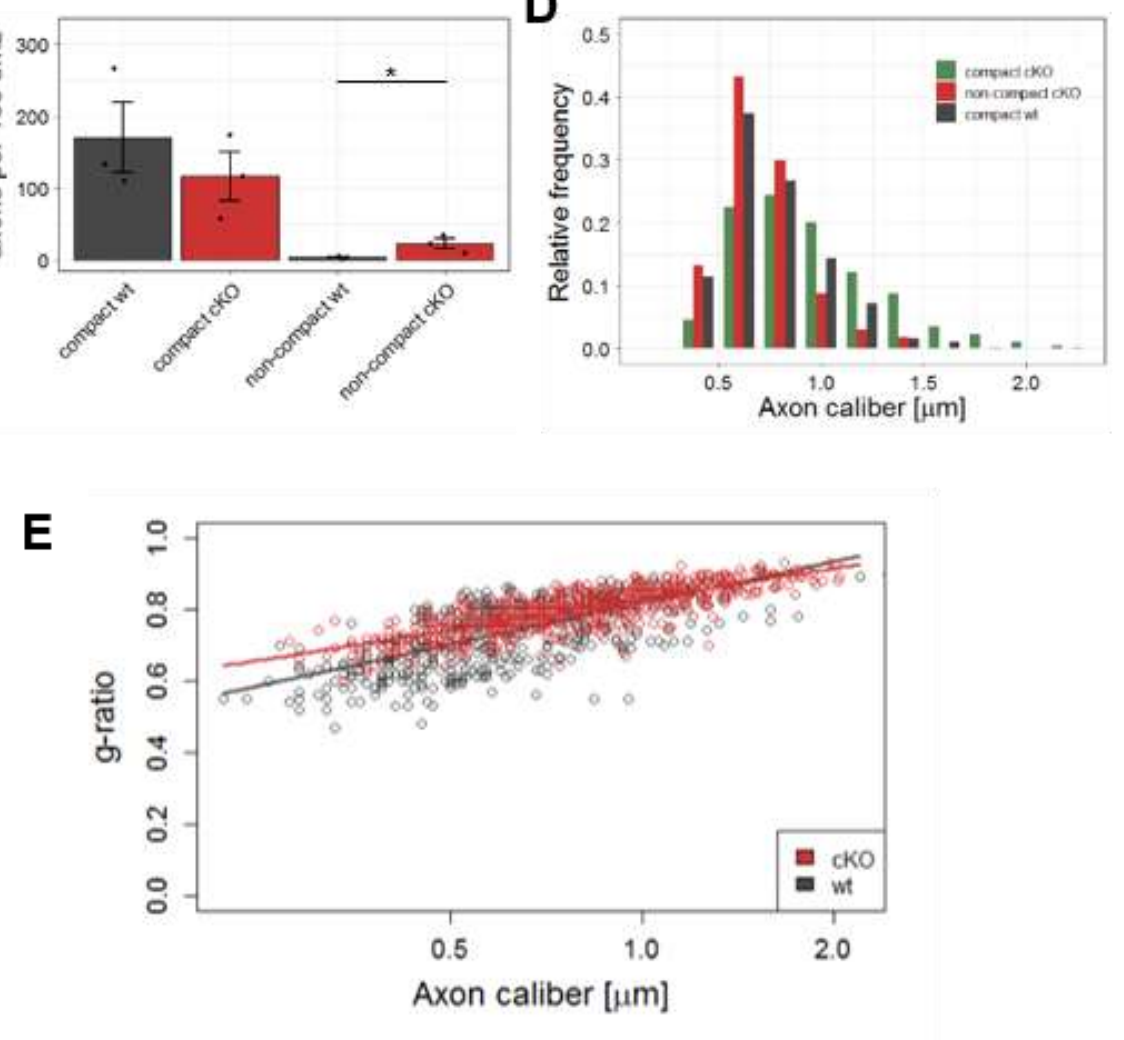
Figure 18: Ultrastructural analysis of the rCC of EMXMBP mice shows a dorsalventral disparity with respect to the dysmyelination phenotype.

A) Representative images of 4-month-old control and cKO EMXMBP mice shows that the ventral $\mathrm{rCC}$ is normally myelinated with the bulk of the dysmyelination observed in the dorsal $\mathrm{rCC}$, inset shows an axon with shiverer phenotype and unmyelinated axons. B) Fraction of axons with compact and shiverer (non-compact) myelin in control (black) and cKO (red) EMXMBP mice. C) Absolute number of axons per $180 \mu \mathrm{m}^{2}$ with compact myelin was unchanged in cKO mice compared to controls but that of shiverer myelin increased in cKO mice, $n=3$ per group, n.s. ( $p$-value $=0.35$ ) and * ( $p$-value $=0.05$ ), respectively, one-sided Wilcoxon rank-sum test. D) Frequency distribution of myelinated axons (both compact and shiverer) in cKO and compact ones in control mice shows a shift in calibre with respect to compactly myelinated axons in the cKO mice (green). In addition, there is a significant increase in smaller calibre axons being shiverer-like in cKO mice (red) compared to control (black) mice. Compact wt: $n=3$, axons $=597$; compact cKO: $n=3$, axons $=616$; non-compact $\mathrm{cKO}: \mathrm{n}=3$, axons $=441$, Pairwise comparisons with two-sided Kolmogorov-Smirnov test, compact wt vs non-compact $c K O: p=0.0003$, compact wt vs compact cKO: $p<1$ e-10, non-compact $c K O$ vs compact $c K O: p<1 e-10$, bin size $=200$ $\mathrm{nm}$. E) G-ratio analysis of myelinated axons in cKO (red) and control (black) mice with respect to axon calibre reveals that small-calibre axons are hypomyelinated in the cKO mice and larger calibre axons are normally myelinated compared to control mice, scale bar $2 \mu \mathrm{m}$, inset $1 \mu \mathrm{m}$. Data acquired and analysed by Jonas Hemesath, Master's student

According to these data, it is clear that there is a successful but partial removal of compact myelin from the corpus callosum. More to the point, the large-calibre axons seem to be normally myelinated whereas the small-calibre axons tend to be more shiverer-like.

Since there is a clear knockout of $M b p$ in the forebrain, I wanted to investigate if this results in any behavioural change.

\subsection{EMXMBP mice show signs of catatonia at 12 months of age}

To investigate whether cortical dysmyelination resulted in behavioural changes (both loss and gain-of-function), female and male EMXMBP mice were exposed to a complex array of behavioural tests that are used to assess many different behavioural domains such as anxiety, social behaviour, sensory and motor function, spatial memory, catatonia and executive function, etc. Sahab Arinrad and Anja Ronnenberg of the Max Planck Institute for Experimental Medicine conducted these behavioural tests and I analysed the data. Both female and male EMXMBP mice performed comparably to control mice in all tests except for the bar test (detailed account of statistical analyses performed for each test is given in Table 9 and Table 10). The bar test is used to assess signs of catatonia, which is 
described as a 'psychomotor syndrome' reflecting a temporary disruption in executive function despite the absence of any obvious motor dysfunction (Janova et al., 2018). In this test, the forelimbs of mice are placed on a bar and the latency of removal of paws from the bar is estimated as the immobility latency. The longer a mouse takes to remove its paws off the bar, the more pronounced the catatonic phenotype it is believed to exhibit. Mice deficient in Cnp, another myelin protein show strong catatonic symptoms (Janova et al., 2018). Female EMXMBP cKO mice, tested at different ages, showed an increased immobility latency compared to controls only at the 11-month time-point (Figure 19 A). Whereas male EMXMBP cKO mice started showing increased latencies starting at 6 months of age until the 1-year time-point (Figure 19 B). However, both female and male cKO mice showed comparable latencies when re-tested at the 17-month time-point.

A

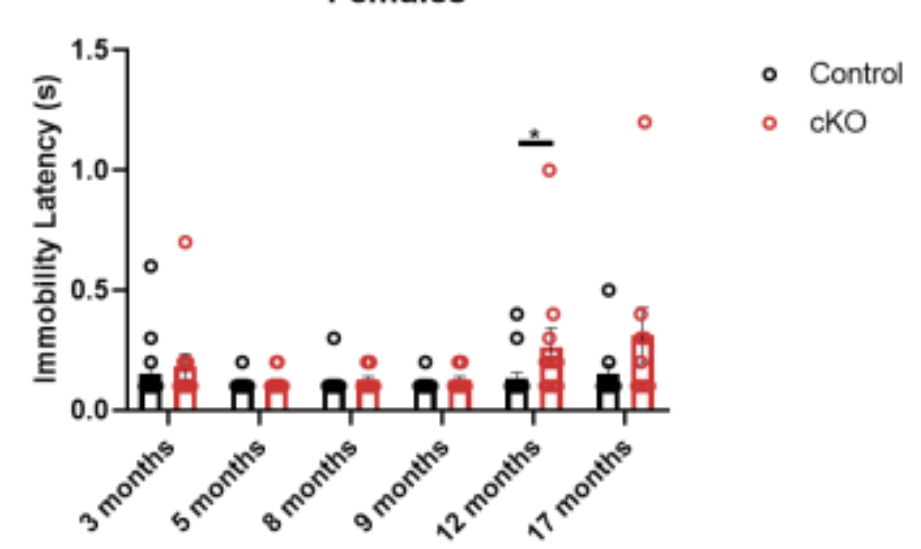

B

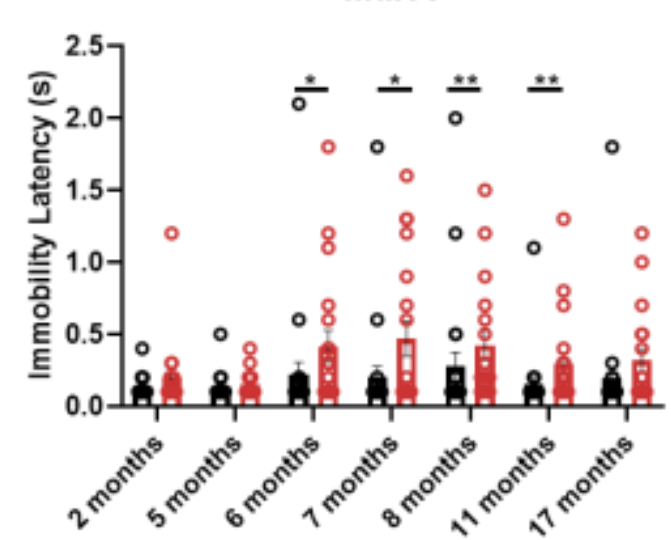

Figure 19: EMXMBP cKO mice spend more time immobile on the bar in the bar test indicative of a catatonia-like symptom.

A) Female EMXMBP mice tested at various time-points. cKO (red) mice exhibit an increased immobility latency at the 1 year time-point compared to control mice (black), * ( $p$-value $=0.0220$, Mann-Whitney test, $n=15$ control and $n=11 \mathrm{cKO})$. B) Male EMXMBP mice tested at various time-points. cKO mice (red) show increased immobility latency starting at 6 months of age until the 11-month time-point, compared to controls (black), ${ }^{*}$ ( $p$-value $(11$ months $)=0.0049$, Mann-Whitney test, $n=23$ control and $n=19 \mathrm{cKO})$. For other $p$-values see Table 9 and 10. Mean \pm S.E.M. data acquisition by Sahab Arinrad and Anja Ronnenberg.

In their study, Janova and colleagues argue that catatonic symptoms, observed in Cnp deficient mice, are ameliorated by ablation of microglia. This means that Cnp deficiency leads to exacerbated inflammation that causes catatonic symptoms. Since I observed an increase in Iba1-positive microglia in the CC of 4-month-old 
EMXMBP mice, it could explain the increased catatonic phenotype. Perhaps the sustained shiverer-like system in the forebrain results in inflammation with time which causes catatonia in these mice. However, this does not explain why there is no catatonia observed in these mice, both male and female, at the 17-month timepoint.

The fact that the ventral CC of the EMXMBP mice is normally myelinated, could potentially account for the lack of behavioural changes seen in the cKO mice. In addition, despite a significant reduction MBP both at the immunohistochemical and at protein levels, some MBP positive OLs could be seen in the CC and the cortex of cKO mice. I postulated that this could be either due to a recombination inefficiency, or infiltration of healthy OPCs from neighbouring regions which differentiated into healthy, myelinating OLs. Hence, we generated the FOXMBP mice. FoxG1 is expressed 4 days prior to Emx1. Hence, we hoped that, as a Cre recombinase-driver, it would be more successful in dysmyelinating the entire CC and cortex.

\subsection{Chracterization of the FOXMBP mice}

\subsubsection{FOXMBP mice have reduced levels of MBP in the forebrain}

As was done previously for the EMXMBP, I validated the knockout of Mbp by quantifying MBP protein levels in the WM, cortex and cerebellum of 6-month-old male cKO and control mice. I found a significant reduction in MBP in the white matter of cKO FOXMBP mice ( $p$-value $=0.0212$, Figure $20 \mathrm{~A}$ ). MBP levels in the cortex were reduced but not significantly compared to controls ( $p$-value $=0.0556$, Figure $20 \mathrm{~B}$ ). This could be possibly due to a high inter-sample variability within the control group. As expected, the cerebellum of both $\mathrm{cKO}$ and control FOXMBP mice has comparable levels of MBP ( $p$-value $=0.5496$, Figure $20 \mathrm{C}$ ).

When I quantified paraffin-embedded sections of 4-month-old male FOXMBP mice, immunostained for MBP, I found a forebrain-specific reduction in MBP intensity in cKO mice compared to controls (Figure $21 \mathrm{~A}$ ). Although there is a reduction in the percentage of area positive for MBP in the CC of cKO mice, it was not significant upon quantification ( $p$-value $=0.0522$, Figure $21 \mathrm{~B}$ ). This could be in part because of inter-sample variability in the control group. There was however, 


\section{Results}

a significant reduction in MBP levels in the cortex of FOXMBP cKO mice ( $p$-value $=0.0027$, Figure $21 \mathrm{C}$ ).

These data confirm that FOXMBP have a reduction in MBP protein in the forebrain.

A

White Matter Lysate

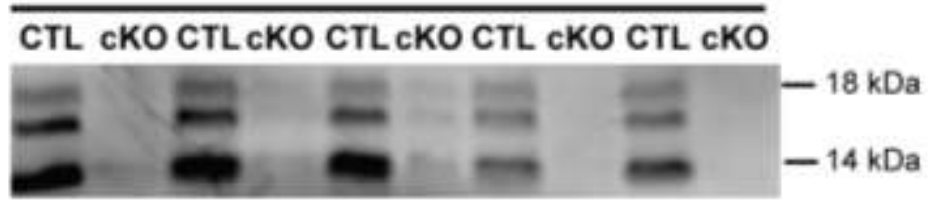

B

Cortex Lysate

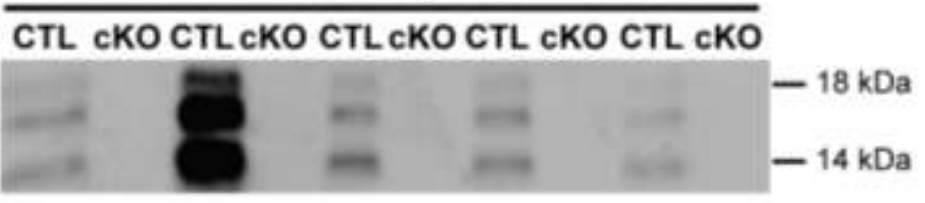

C

Cerebellum Lysate

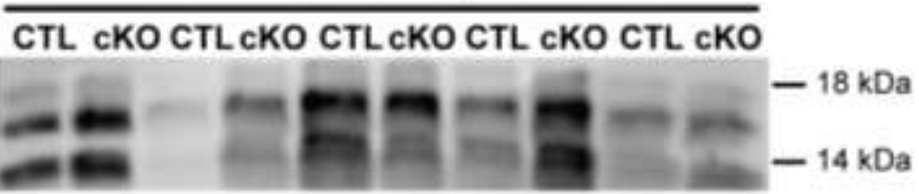

White Matter

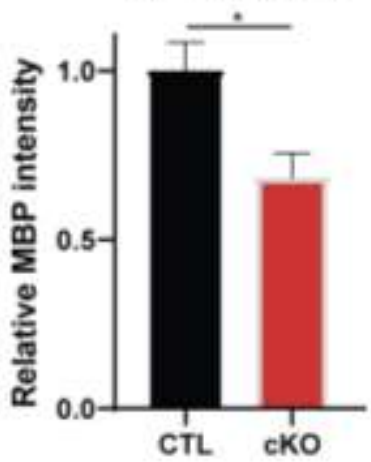

Cortex

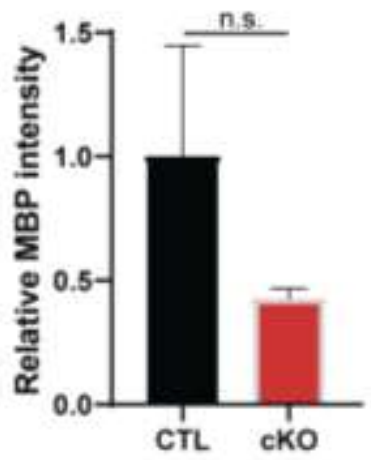

Cerebellum

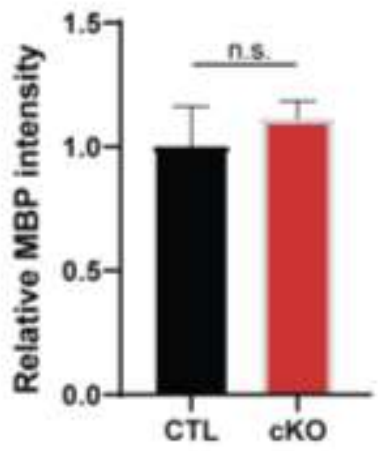

Figure 20: Protein estimation of MBP in FOXMBP shows a reduction in protein levels in the forebrain.

A) MBP levels in the white matter lysate of male cKO FOXMBP (red) mice are significantly less compared to controls (black), ${ }^{*}(p$-value $=0.0212)$. B) MBP levels in the cortex of CKO FOXMBP mice are reduced but not significantly compared to control, n.s. ( $p$-value $=0.0556$ ). C) MBP levels in the cerebellum of cKO FOXMBP mice remain the same compared to control, n.s. ( $p$-value $=0.5496)$. Two-tailed student's t-test with Welch's correction, $n=5$ for both groups. Mean \pm S.E.M. 
A
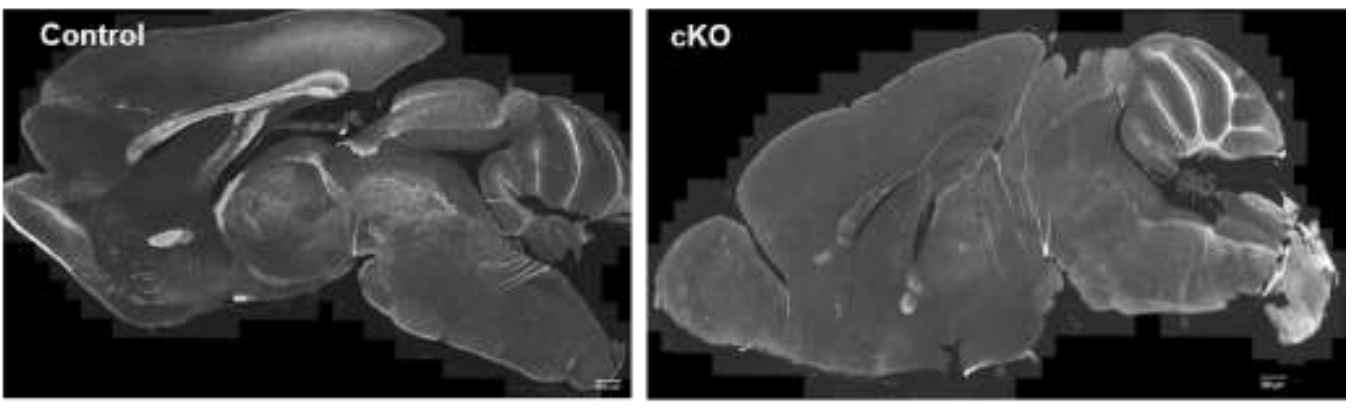

B

Corpus Callosum

C

Cortex
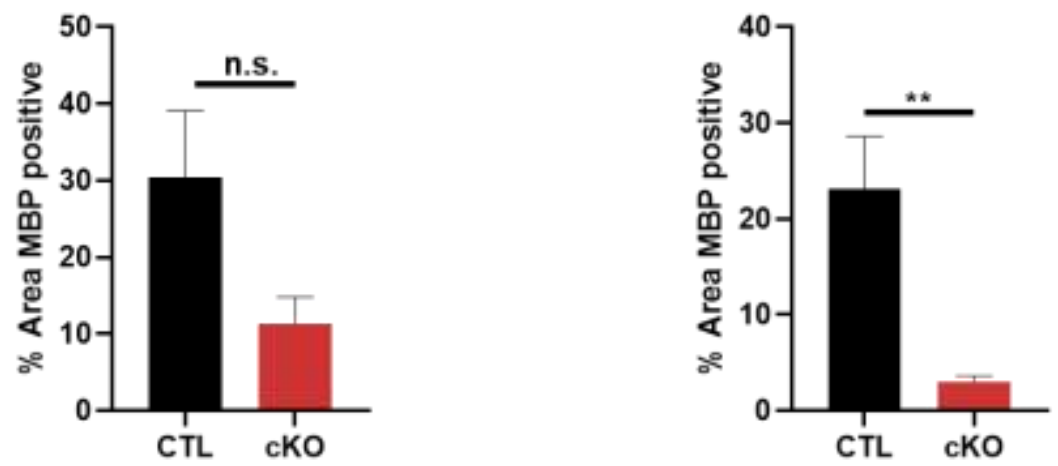

Figure 21: Immunohistochemical quantification of MBP shows a reduction in FOXMBP mice.

A) representative images of sigittal sections $(5 \mu \mathrm{m})$ of 4 -month-old male control and cKO mice stained for MBP shows a remarkable reduction in MBP only in the cKO forebrain. Other structures are well preserved with respect to MBP expression. B) Quantification of the percentage of area positive for MBP in the CC shows a reduction, but not a significant one, of MBP in the cKO mice (red) comapred to control mice (black), n.s. ( $p$-value $=0.0522$ ). C) Quantification of percentage of area positive for MBP in the cortex shows a significant reduction in MBP in the cKO mice compared to control mice, ${ }^{* *}(p=0.0027)$. Unpaired two-tailed student's t-test, $n=3$ control and $n=$ $5 \mathrm{cKO}$, Mean \pm S.E.M., scale bar $500 \mu \mathrm{m}$.

\subsubsection{FOXMBP mice show a mild form of gliosis in the corpus callosum}

To check for gliosis in the CC and cortex of FOXMBP mice, paffin-embedded brain slices of 4-month-old mice were stained for GFAP, Iba1, Mac3, CD3 and APP (Figure 22 F). There was a significant increase in astrogliosis in both CC and cortex of cKO FOXMBP mice compared to controls ( $p$-value $=0.0095$, CC; $p$-value $=0.0238$, cortex, Figure $22 \mathrm{~A}$ ). There were also more microglia in the cKO CC compared to controls ( $p$-value $=0.0065)$ but not in their cortex ( $p$-value $=0.2790$ )

(Figure 22 B). These microglia seemed to be significantly more reactive in the CC $(p$-value $=0.0317)$, but not in the cortex $(p$-value $=0.2058)$ of the cKO mice 


\section{Results}

(Figure $22 \mathrm{C}$ ). Number of CD3 positive cells were comparable both in the CC ( $\mathrm{p}$ value $=0.8553)$ and cortex ( $p$-value $=0.8264)$ of the cKO mice suggesting an intact and functional bloood-brain barrier (Figure $22 \mathrm{D}$ ). No axonal degeneration was observed in the CC ( $p$-value $=0.3659)$ or the cortex $(p$-value $=0.3748)$ of cKO mice, visualised by counting the number of APP swellings in these regions (Figure $22 \mathrm{E})$.

Despite showing mild gliosis in the CC and cortex at 4 months of age, I concluded that overall the neuropathology in the FOXMBP mice is normal. However here, like the EMXMBP mice, I saw an increased number of cells independent of microglia and astrocytes. To investigate whether the FOXMBP also have increased OPCs and OLs in the CC and cortex, I quantified their numbers. 
A
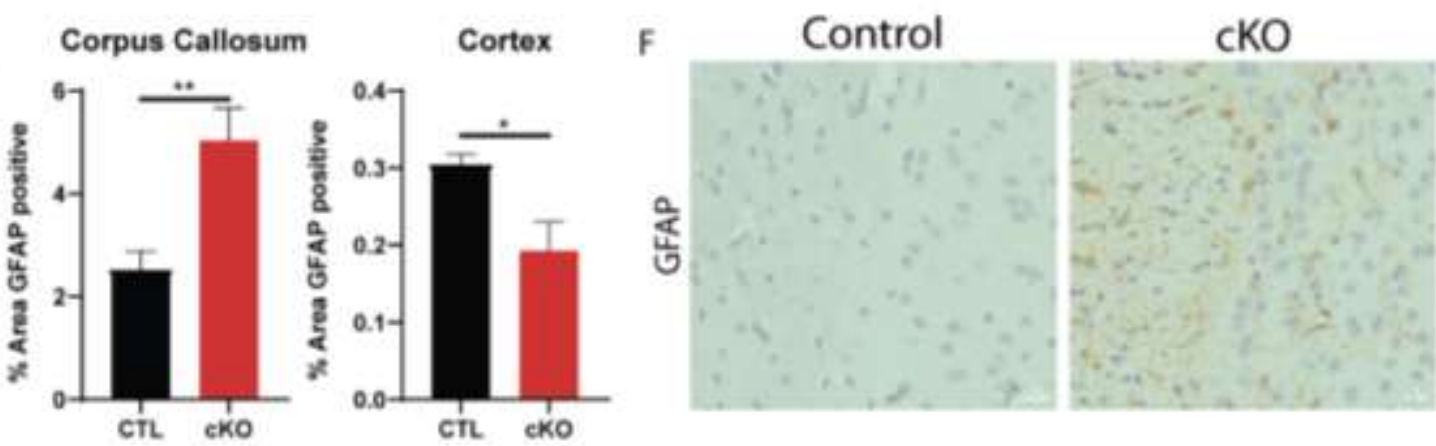

B
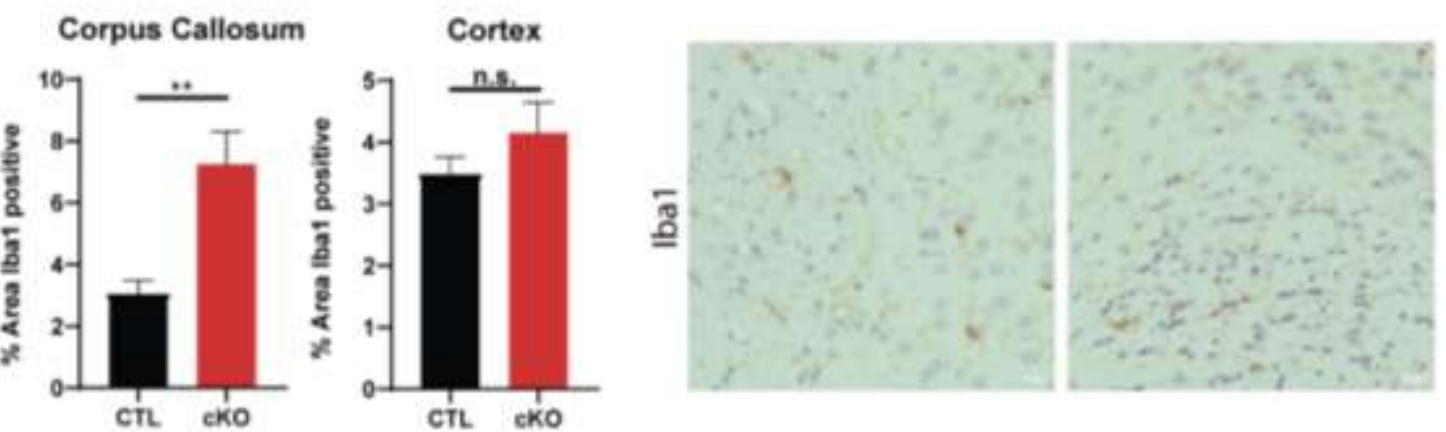

C

Corpus Callosum

Cortex
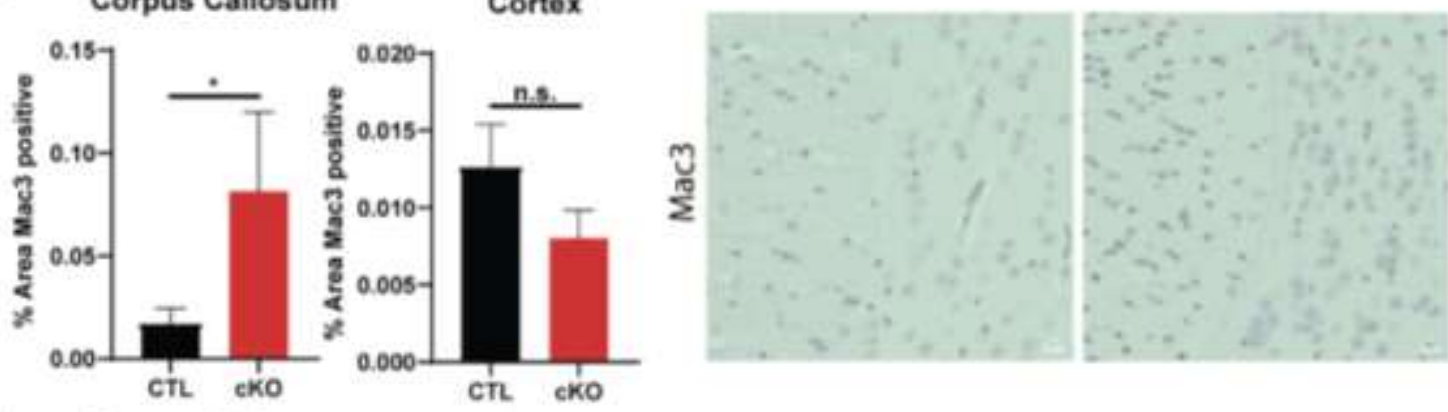

D

Corpus Callosum

Cortex
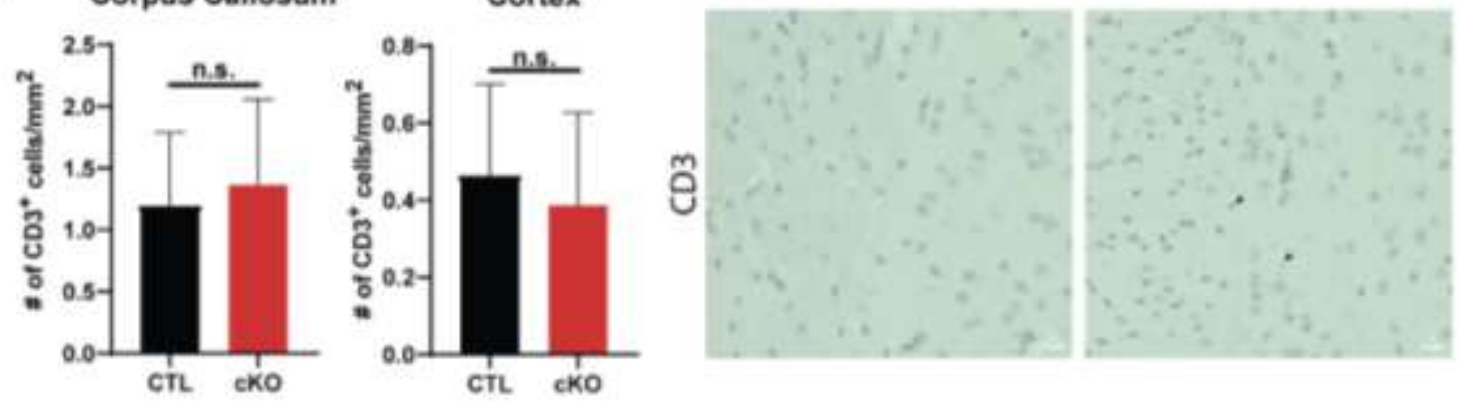

E
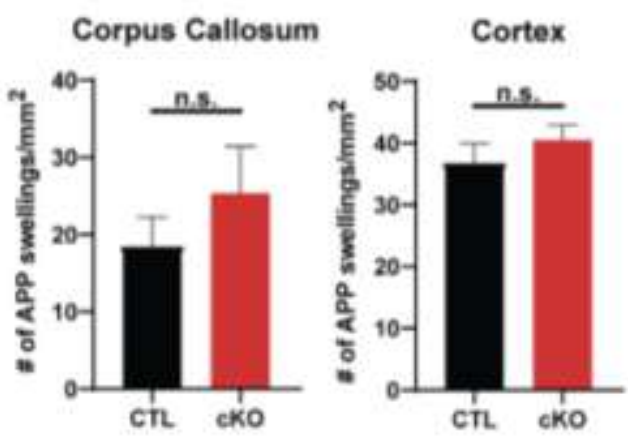

$\frac{0}{4}$

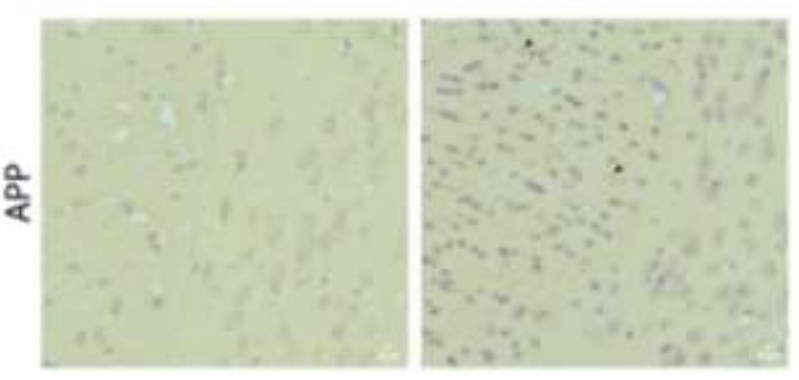


Figure 22: FOXMBP mice show a mild form of gliosis in the corpus callosum at 4 months of age.

A) Percentage of area positive GFAP was slightly elevated in the CKO CC and cortex, ** $(p$-value $=0.0095, C C),{ }^{*}(p$-value $=0.0238$, cortex $\left.) . B\right)$ Percentage area positive for lba1 was increased in cKO CC but not in the cortex, ${ }^{* *}$ ( $p$-value $\left.=0.0065, C C\right)$, n.s. $(p$-value $=$ 0.2790 , cortex). C) Percentage area positive for Mac3 was was slightly elevated in the CC but unchanged in the cortex of cKO mice, ${ }^{*}$ ( $p$-value $\left.=0.0317, C C\right)$, n.s. ( $p$-value $=0.2058$, cortex). D) Number of CD3 positive cells was unchanged in both CC and cortex of the cKO mice, n.s. ( $p$-value $=0.8553, \mathrm{CC} ; 0.8264$, cortex). E) Number of APP swellings were comparable in both CC and cortex of cKO mice, n.s. ( $p$-value $=0.3659, C C ; 0.3748$, cortex). F) Respresentative images of the $\mathrm{CC}$ and cortex of 4-month-old $\mathrm{cKO}$ and control FOXMBP mice stained for GFAP, Iba1, Mac3, CD3 (arrowheads indicate CD3 positive cells) and APP (arrowheads indicate APP swellings), respectively. Unpaired two-tailed student's t-test or Mann-Whitney test, $n=5$ for both groups Mean \pm S.E.M., scale bar 5 $\mu \mathrm{m}$. Data acquired and analysed by Jonas Hemesath, Master's student.

\subsubsection{There are more OPCs and OLs in the corpus callosum of FOXMBP mice}

I stained paraffin-embedded slices of 6-month-old FOXMPB mice for Olig2 and CAll to quantify the density of OPCs and OLs in the CC and cortex (Figure 23 A and $D$, respectively). I found a significant increase in the number of OPCs and OLs in the CC of cKO mice compared to controls ( $p$-value $=0.0409$, OPCs; 0.0415 , OLs, Figure $23 \mathbf{B}$ and $\mathbf{C}$ ). However, there was no significant increase in the number of OPCs and OLs in the cortex ( $p$-value $=0.5062$, OPCs; 0.2752 , OLs, Figure $23 \mathrm{E}$ and $\mathrm{F}$ ).

These data suggest that there is an increase in the number of OPCs and OLs in the CC of FOXMBP mice but not in their cortex. Since the CC is most extensively myelinated in the CNS, it makes sense that more OPCs are recruited in the absence of compact myelin to ameliorate its deficiency. However, the lack of compact myelin in the cortex does not seem to trigger the same, perhaps due to the heterogeneous nature of myelination there. 
A

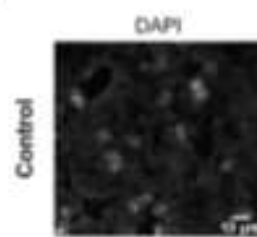

9
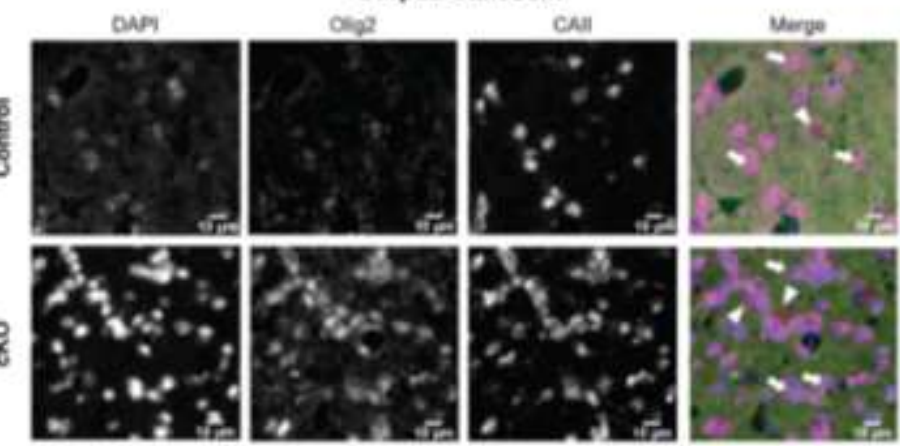

B

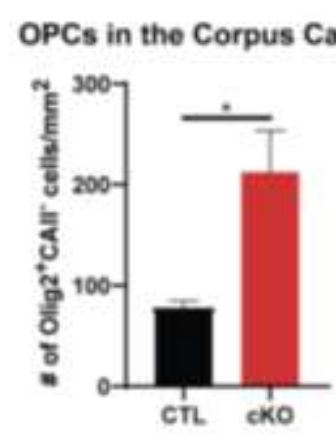

c

OLs in the Corpus Callosum

D
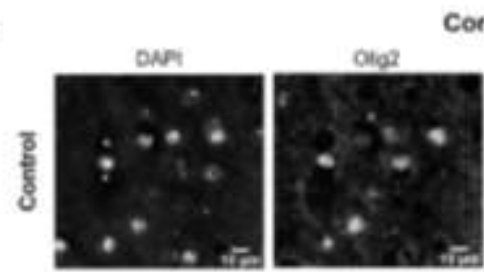

Cortex
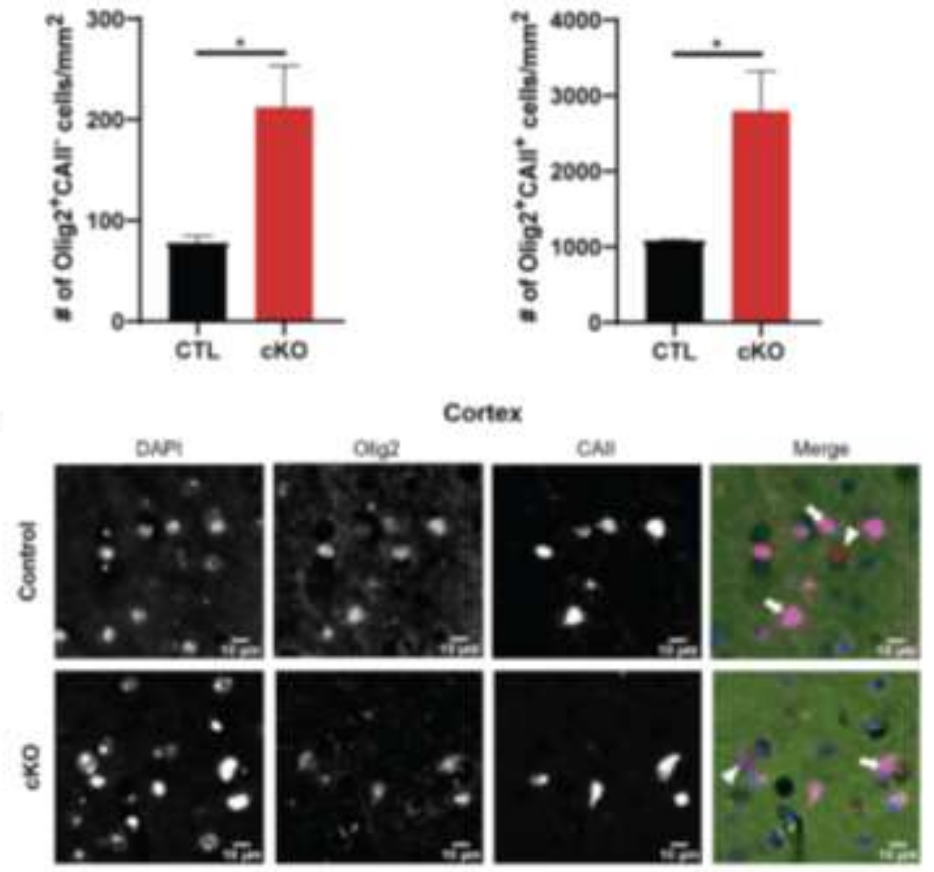

E

OPCs in the Cortex

F

OLs in the Cortex
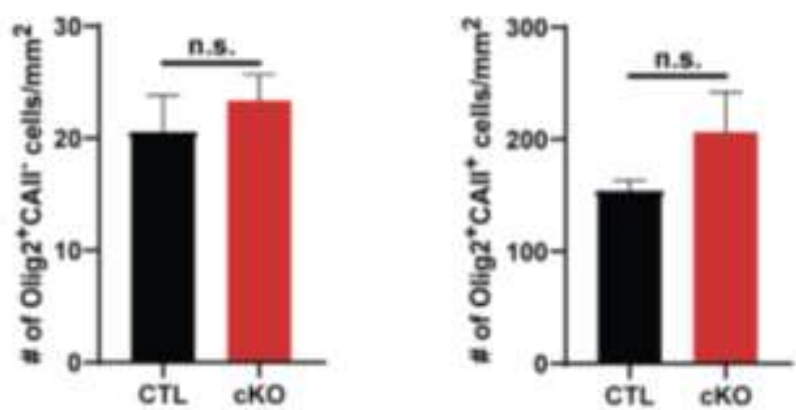

Figure 23: FOXMBP mice have more OLs in the CC.

A) and D) Representative images of OPCs and OLs in CC and cortex of 6-month-old FOXMBP cKO and control mice. B) FOXMBP cKO mice have significantly more OPCs in the CC, ${ }^{*}(p$-value $=0.0409)$. C) The CC of FOXMBP cKO mice (red) has significantly more OLS compared to controls (black), ${ }^{*}(p$-value $=0.0415)$. E) Number of OPCs in the cortex of FOXMBP cKO mice is comparable to controls, n.s. (p-value $=0.5062)$. F) No increase in the number of OLs in the cortex is observed in the cKO FOXXMBP mice compared to controls, n.s. ( $p$-value $=0.2752$ ). Unpaired two-tailed student's t-test, $n=3$ control and $4 \mathrm{cKO}$, Mean \pm S.E.M., scale bar $10 \mu \mathrm{m}$. 


\subsubsection{The rostral corpus callosum of FOXMBP mice is almost entirely dysmyelinated}

On assessing the ultrastructure of the CC of FOXMBP mice, it seemed that the rCC of CKO mice was almost entirely Dysmyelinated. Whereas, the caudal CC (cCC) was normally myelinated similar to control mice (Figure $24 \mathrm{~A}$ ). Hence, all analysis was done in the rCC. On comparing the fraction of axons exhibiting normal compact and shiverer-like myelin phenotype, it appeared that almost the entire rCC of cKO mice consisted of shiverer-like axons, compared to control mice (Figure 24 B). Looking at the absolute number of myelinated axons per $180 \mu \mathrm{m} 2$, it was even more apparent that there is a significant reduction in normally myelinated fibres $(p$ $=0.02487)$ and an increase in fibres with shiverer-like, non-compact myelin ( $p=$ 0.02857 ) between the rCC of control and cKO mice (Figure $24 \mathrm{C}$ ). In addition, the relative distribution of myelinated axon calibres is slightly but significantly shifted, in the rCC of CKO mice, with more large-calibre axons possessing shiverer-like myelin ( $p$-value $=0.007802$, Figure $24 \mathrm{D}$ ). Since there are not many axons with compact myelin in the rCC of cKO mice, calculation of g-ratio was not possible for them (Figure $24 \mathrm{E}$ ).

These data indicate that while the extent of dysmyelination is more pronounced in the FOXMBP mice, a complete knockout of $\mathrm{Mbp}$ was not achieved since the $\mathrm{CCC}$ is normally myelinated in the cKO mice. 

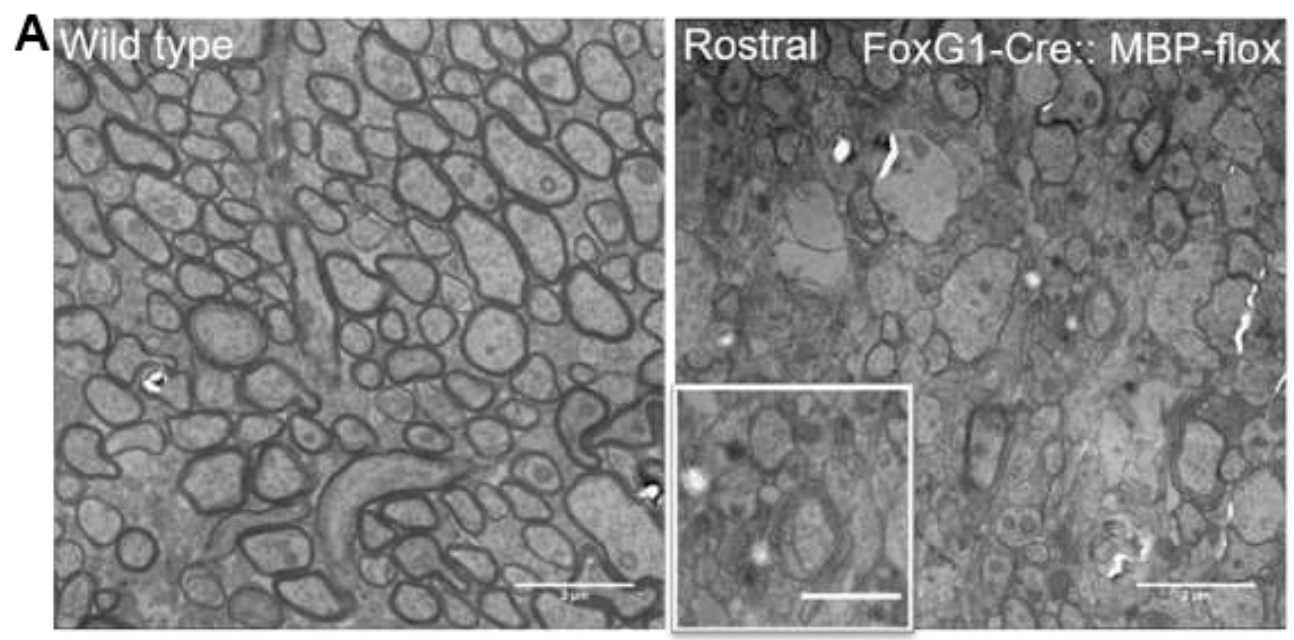

B
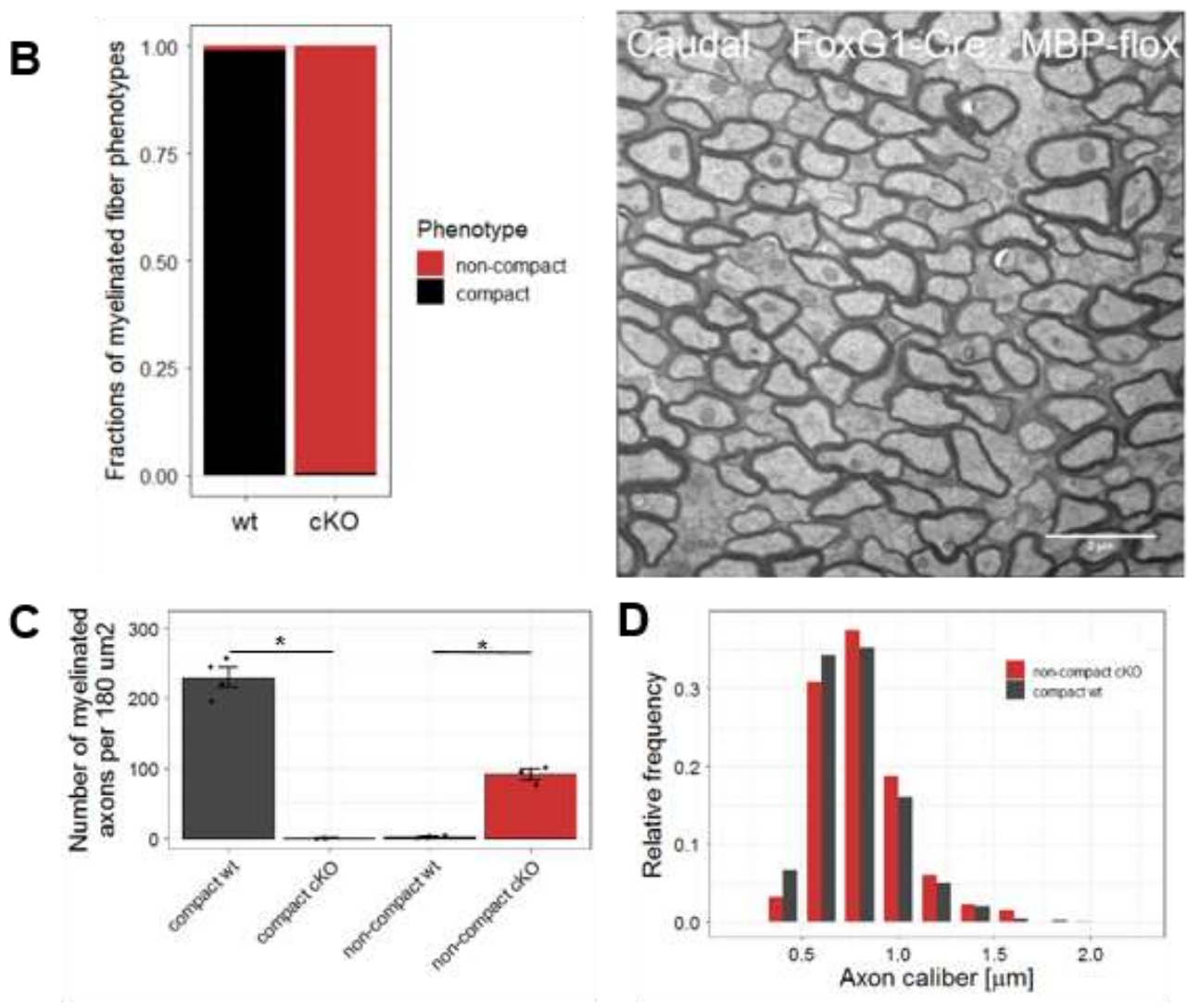

E

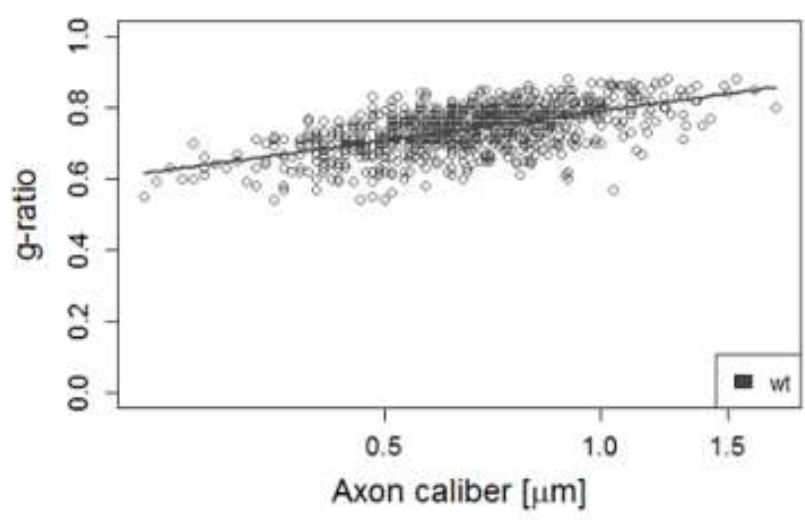


Figure 24: The rCC in FOXMBP mice is almost entirely dysmyelinated whereas the $\mathrm{CCC}$ is comparable to control mice.

A) Representative EM images of the CC of 4-month-old FOXMBP mice shows a rostral-caudal disparity with respect to myelination. The $\mathrm{CCC}$ is normally myelinated in cKO mice. Due to this only the rCC was compared in cKO and control mice, inset shows shiverer-like axons. B) Comparison of the fraction of myelinated axons in the $\mathrm{rCC}$ of control (black) and cKO (red) mice shows a remarkable reduction in compact myelin in the cKO. C) Absolute number of axons per $180 \mu^{2} \mathrm{~m}^{2}$ with compact myelin was significantly reduced and that of shiverer myelin was increased in in cKO mice compared to controls, $n=4$ control, $n=3 \mathrm{cKO},{ }^{*}(p=0.02487$, compact myelin and $p$ $=0.02857$, shiverer myelin), one-sided Wilcoxon rank-sum test. D) Frequency distribution of axon-calibres with compact and shiverer myelin in control (compact-wt) and cKO (non-compact cKO) mice, respectively, reveals a slight shift with respect to cKO mice. ${ }^{* *}$ ( $p$-value $=0.007802$, compact wt: $n=4$, axons $=827$; non-compact cKO: $\mathrm{n}=3$, axons $=583$, two-sided Kolmogorov-Smirnov test). E) g-ratio distribution with respect to axon calibre in FOXMBP mice $(n=4$, axons $=827$, control). Since the cKO rCC has only shiverer axons, g-ratio estimation was not possible. Bin size: $200 \mathrm{~nm}$, scale bar $2 \mu \mathrm{m}$, inset $1 \mu \mathrm{m}$. Data acquired and analysed by Jonas Hemesath, Master's student.

\subsubsection{Mice lacking compact myelin the in forebrain show no behavioural lateralization impairment}

The role of CC in establishment of lateralized functions is long disputed among researchers. While some studies support and hence, propose that the CC is crucial for the development of functional asymmetry (Cassells et al., 1990), others argue on the mechanism underpinning this theory (van der Knaap and van der Ham, 2011).

Since the decision-making and execution of using a forelimb preferentially requires interaction between different specialized regions of the cortex, it is crucial to address the role cortical myelination plays in establishment of handedness. Moreover, it is not only the interactions between cortical regions within the same hemispheres, that determine handedness, but also interhemispheric transfer across the CC that may be important.

To address this, I tested both female and male FOXMBP mice at 10 weeks of age for two consecutive days ('training' and 'test' day). The 'test' day was used for statistical analysis. The conditional knockout (cKO) mice are normally lateralized at 10 weeks ( $p$-value $=0.6214$, Figure $25 \mathrm{~A}$ ). In addition, they exhibit a U-shaped distribution curve with respect to handedness at this time-point with a slightly higher tendency towards being right-handed on the population level (Figure 25 B). 
Previously, data from the lab also showed that 13-week old EMXMBP mice, also tested once, show no impairment in behavioural lateraliazation. Morphological characterization of this line shows that the ventral CC of these mice is normally myelinated. This suggested that perhaps partial myelination of the $C C$ is sufficient for the normal establishment of handedness. Since the FOXMBP mice, in turn, have a normally myelinated caudal CC and show unimpaired behavioural lateralization as well, I concluded that I could not address the specific role of CC in establishment of paw preference with these mouse lines. In addition, the heterogeneity of cortical myelination further calls into question whether cortical dysmyelination severely affects behavioural lateralization.

A Strength of Lateralization

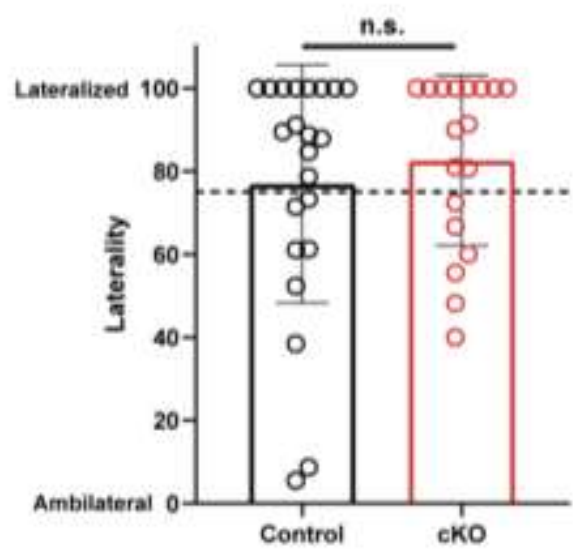

B

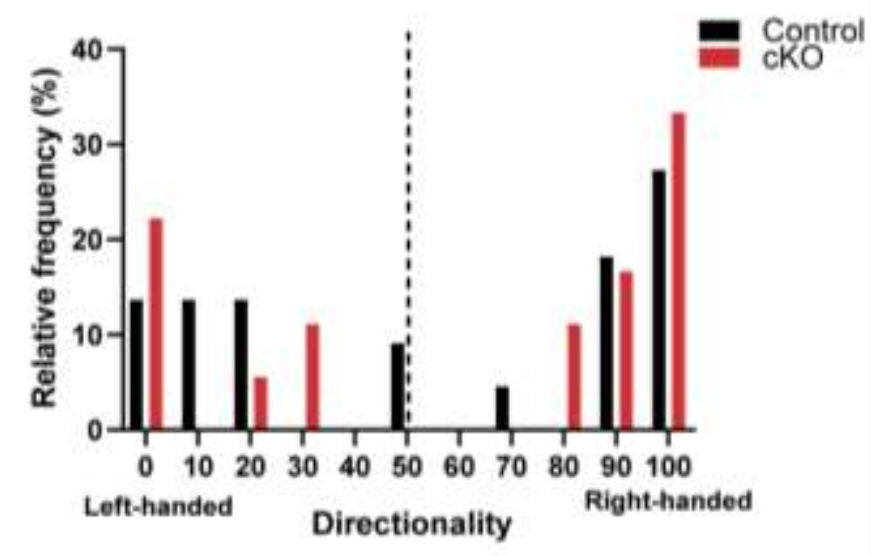

Figure 25: Mice lacking compact myelin in the forebrain do not show impairment of behavioral laterality.

A) FoxG1Cre::Mbpflox mice were tested at 10 weeks of age and were normally lateralized compared to control mice, $\mathrm{n}=22$ control (black) and $\mathrm{n}=18 \mathrm{cKO}$ (red), n.s. ( $p$-value $=$ 0.6214), Mann-Whitney test. B) Relative frequency distribution of handedness of FoxG1Cre::Mbpflox at 10 weeks of age shows the U-shaped distribution of both cKO and control mice. The cKO mice show a tendency towards being right-handed. Bin size $=10$. 


\section{Discussion And Conclusions}

\section{Discussion and Conclusions}

\subsection{Is there a critical period for the establishment of handedness and does myelin play a role in it?}

Critical periods have been described for many systems such as the visual system (Hubel and Wiesel, 1970), the auditory system (Kral, 2013; Sharma et al., 2002), language learning in humans (Francis, 2005). Recent studies have implicated the role of myelin in critical period closure (McGee et al., 2005; Stephany et al., 2014). In humans, handedness is often unstable in infancy, although it has been observed in foetuses at gestation week 15 with an acute preference for thumb-sucking (Hepper, 2013; Hepper et al., 1991). Indeed a study, later, in 2005 found that children tested for handedness at 10-12 years of age showed a bias similar to the one they showed in the womb with respect to thumb-sucking (Hepper et al., 2005). In mice handedness depends on an intact corpus callosum (Preuss et al., 2002), a fibre path that is only fully myelinated in 8-month old adult mice (Sturrock, 1980). Drawing on these observations, I wanted to address the question of when handedness develops and whether a critical period for this establishment can be outlined. For this, I turned to the paw preference paradigm of behavioural laterality, developed in the 1960s by Robert Collins (Collins, 1968, 1969b, 1975). I assessed paw preference in wild type C57BL/6J mice starting at 4 weeks of age and tested them on a weekly basis until 8 weeks of age. I found that wild type mice tested weekly progressively established a paw preference by 8 weeks of age (Figure 7) and showed a typical U-shaped population distribution with respect to the direction of handedness (Figure 8). This led me to conclude that the critical period for the establishment of paw laterality, in wild type mice, is between 4 and 8 weeks of age. Since peak of myelination also coincides with this time-period, I decided to address the role myelination plays in this establishment. I adopted the shiverer mouse model of overall dysmyelination. Based on pervious data from the lab (Moore et al., in preparation), it has been shown that shiverer mice, tested above the age of 8 weeks, exhibit a reduced paw lateralization compared to wild type mice. Hence, I tested shiverer mice along with littermate control mice on a weekly basis between 4 and 9 weeks of age. I called this cohort the 'weekly' cohort and was intrigued to 


\section{Discussion And Conclusions}

find out that young shiverer mice, tested repetitively, were comparably lateralized to control mice by 9 weeks of age (Figure 9). In addition, they showed a U-shaped population distribution of handedness similar to wild type mice. However, this handedness was unstable and prone to changing between tests, especially for shiverer mice (Figure 10).

I postulated that this could either be due to practice, a consequence of repetitive testing, or dysmyelination or developmental plasticity. To address these hypotheses, I tested shiverer mice once at 4 weeks of age, did not test them until 8 weeks and then tested them repetitively until 10 weeks of age. I named this cohort the 'spaced' cohort. My line of thinking was as follows: If the shiverer mice tested, again, at 8 weeks of age continued to show a reduction in paw lateralization that increased with repetitive testing until 10 weeks, the effect I see in the weekly cohort may be predominantly due to repetition. Moreover, an extended plasticity for learning due to dysmyelination allows shiverer mice to become lateralized with practice, irrespective of age. Shiverer and control mice from the spaced cohort are comparably lateralized at the 8-week time-point (Figure 11). In addition, these shiverer mice tend to be less unstable compared to shiverer mice from the weekly cohort and mice that are considered unstable are more likely show the switch in handedness between the 4- and 8-week time-points rather than the later timepoints (Figure 12). I also compared the strength of lateralization of both cohorts at the 4- and 8- week time-points and found no difference between the 8-week timepoints of both cohorts (Figure 26). At this point, it is important to point out that, wild-type littermates from both shiverer cohorts also seemed to be more lateralized at the 4-week time-point compared to the pure wild-type mice (Figure 7). Even though the mice exhibited no abnormal behaviours, it is not possible to account for any effect that could be due to the inheritance of the wild-type allele from parents that carried the mutation. I could not find a sufficient justification that would explain the increased lateralization. However, this argues in favour of the requirement of such high sample numbers. This helps weed out anomalies that may go unnoticed in small cohort numbers. Even if, here, it is in the case of wild-type littermate mice. 


\section{Discussion And Conclusions}

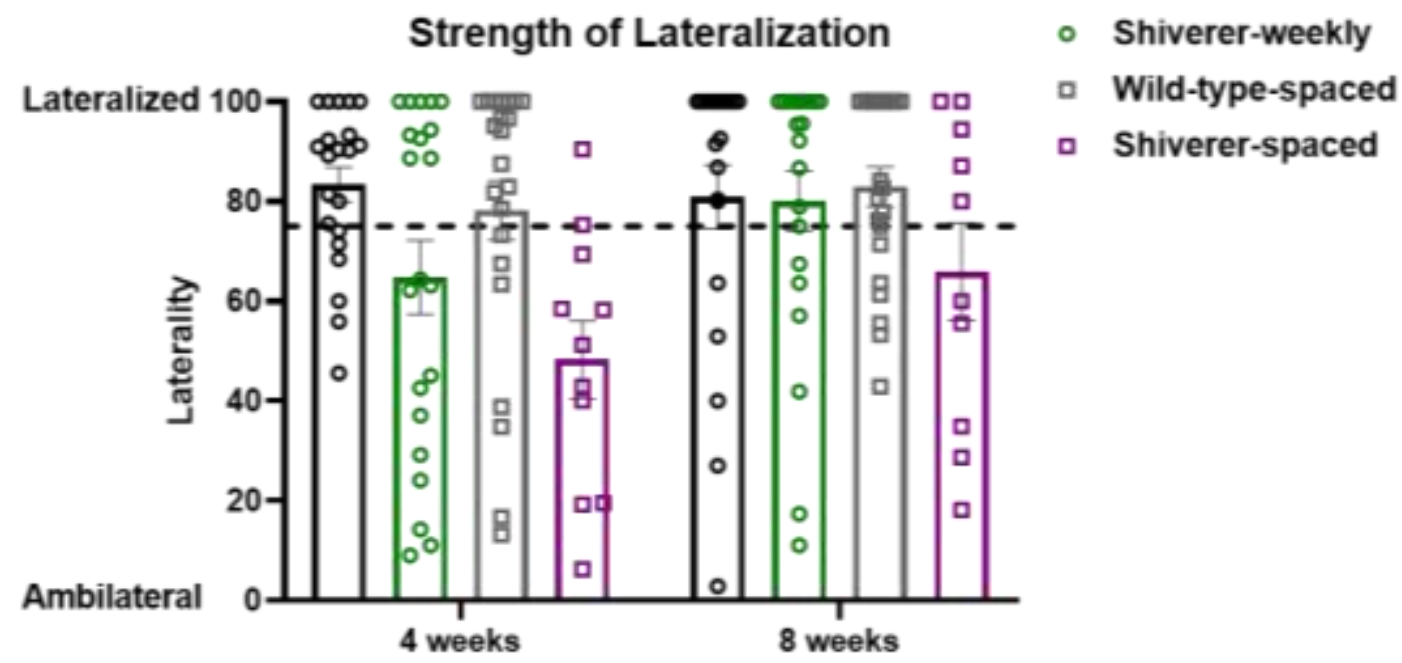

Figure 26: Strength of lateralization at 4 and 8 weeks of age for both weekly and spaced cohorts of shiverer mice.

Comparison between the laterality of shiverer and control mice at the 4- and 8-week time-points blonging to the weekly and spaced cohorts reveals that there is no difference between shiverer and control mice from the two cohorts at 8 weeks of age, n.s., mixed effect two-way ANOVA with Tukey's multiple comparison test, $n=21$ both shiverer and wildtype - weekly, $\mathrm{n}=22$ control and $\mathrm{n}=12$ shiverer - spaced.

With these data, I concluded that the instability observed in both shiverer and wild type mice from both cohorts may be a consequence of developmental plasticity. The effect of practice is apparent in both weekly and spaced cohorts, with a reduction in lateralization being observed only at the 4-week time-point. It has been previously reported that repetition of the paw preference task has an effect on the laterality (Bulman-Fleming et al., 1997; Collins, 1975). Bulman-Fleming and colleagues in particular, assessed the importance of the order to which mice were introduced to the paradigm. They proposed that mice first tested in a session consisting of multiple reaches and then exposed to sessions that were shorter (consisting of a single reach) but occurred on a weekly basis, tended to show a significantly greater increase in behavioural laterality compared to those exposed to the reverse order. This means, naivety with respect to this behavioural paradigm strongly influences the outcome of paw lateralization in mice and that repetition reinforces the lateralization, thereby increasing paw laterality scores in these mice. 


\section{Discussion And Conclusions}

To control for the apparent issue of naivety, I would have had to test a separate, naïve cohort of shiverer and control mice at each time-point. Since to get a reliable readout of behavioural lateralization, I require at least 20 mice per group, this would not have been feasible.

Lateralization in wild type adult mice is fixed once established and does not change over time (Moore et al., in preparation). My data in young wild type mice reflects a period during which mice progressively develop a paw preference and that by 8 weeks of age they are lateralized. I tested young shiverer mice to elucidate the role of myelination in the establishment of paw lateralization. I found that young shiverer mice, when tested repetitively, become lateralized compared to shiverer mice trained only in adulthood. Moreover, the direction of handedess exhibited by these young shiverer mice is unstable and prone to changing between tests. These data suggest that in the absence of compact myelin, paw preference is established albeit unstable in mice.

A couple of recent studies conducted to determine the handedness of marsupial animals showed interesting results. Marsupial animals are of relevance here because they lack a corpus callosum. Giljov and colleagues conducted two separate studies where they assessed the paw preference of bipedal marsupial animals such as kangaroos and wallabies (Giljov et al., 2015) and quadrupedal marsupial animals such as grey short-tailed opossum and sugar gliders (Giljov et al., 2013) with respect to some unimanual tasks such as feeding. They found that bipedal marsupials show a strong manual lateralization, interestingly leftwards, compared to quadrupedal marsupials in most unimanual tasks. They argued that bipedal marsupial exhibit 'true handedness' which may be a product of their postural evolution, leading to the specialization of their forelimb and its eventual lateralization. I decided to compare my data from mice and the marsupial data. I found that my mice show a similar distribution to that exhibited by sugar gliders (Figure 27). Interestingly, in our hands (Moore et al, in preparation) adult shiverer mice are rarely lateralized but when they are, they tend to be left handed. Shiverer mice, therefore, show a pattern that is a mixture of bipedal and quadrupedal marsupials, consistent with the absence of a functional CC. This begs the question if one accepts the claim that 'true handedness' is exhibited by species that evolved 


\section{Discussion And Conclusions}

to specialize their forelimbs for unimanual tasks and whether mice that use both paws to retrieve food for the most part in the wild even require a paw preference. Since marsupial animals belong to the noneuthrian group, they too lack a CC (Suárez et al., 2018). In this light, it is intriguing to postulate what the role of interhemispheric transfer of information, via the $\mathrm{CC}$, really is in the development of handedness. Did the evolution of the CC contribute to the lateralization of handedness? Alternatively, was there a concomitant effect of humans and other non-human primates adapting bipedal postures and the increased interhemispheric transmission, which led to the kind of handedness observed today?

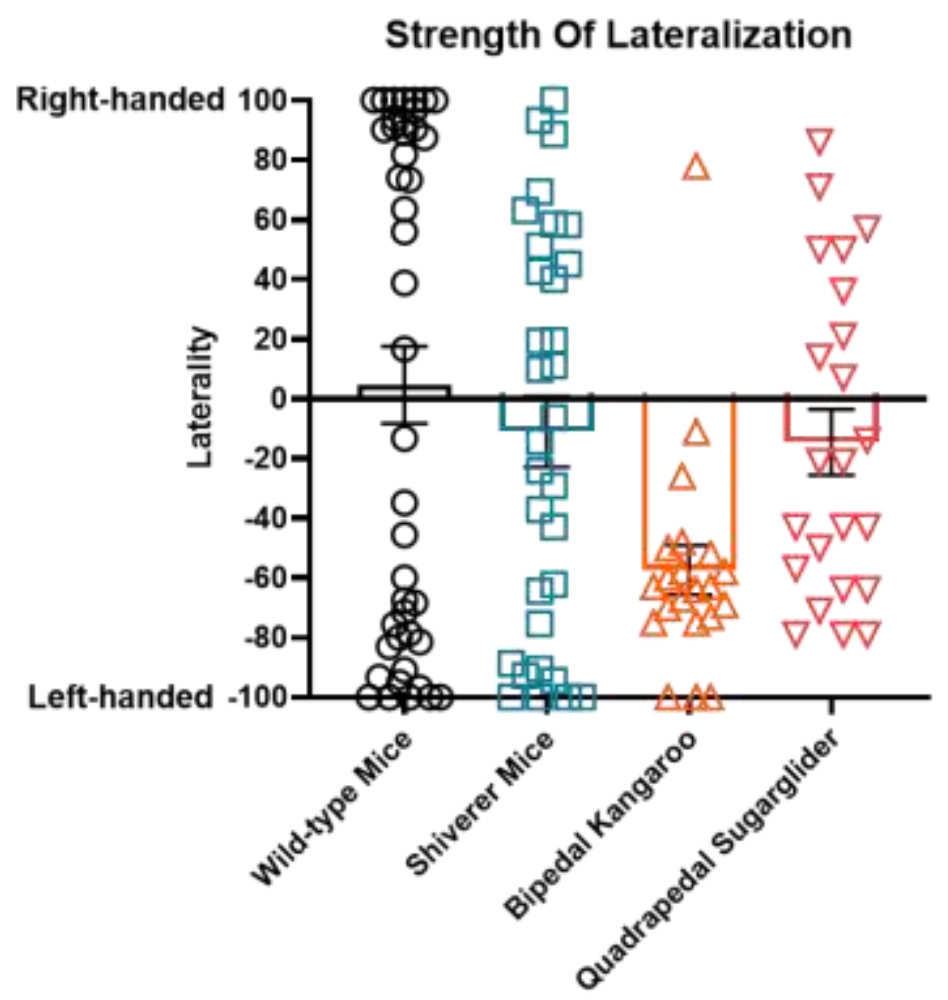

Figure 27: Strength of lateralization of mice and bipedal and quadrupedal marsupial animals.

Comparison of laterality of shiverer, control mice, bipedal and quadrapedal marsupials such as the red kangaroo (Macropus Osphranter rufus) and the sugra glider (Petaurus breviceps), respectively. It reveals that red kangaroos show a strong leftward bias with respect to handedness. The food eating behaviours of all animals were compared. *** ( $p$-value $=0.0003)$, Welch's ANOVA W(7.376) with Holm-Sidak's multiple comparison test, $n=43$ wild type mice, $n=33$ shiverer, $n=21$ red kangaroos and $n=23$ sugar gliders. Sugar glider data collected from Giljov et al., 2013 and red kangaroo data collected from Giljov et al., 2015. 


\section{Discussion And Conclusions}

\subsection{Characterization of mice lacking compact myelin in the forebrain}

Shiverer mice lack compact myelin in their entire CNS, which on one side result in motor deficits that complicate cognitive tests and, on the other hand, make it difficult to draw conclusions about the role of myelin in higher cognitive processes. To study the role of cortical myelination in higher brain functions it was necessary to generate a mouse model, which could help pinpoint the role of cortical myelination alone and not show secondary behavioural deficits due to dysmyelination in other regions of the CNS. Hence, two mouse models were generated, to this effect, showing variable extents of dysmyelination. The Emx1IRES-Cre:: Mbp flox (EMXMBP) mice show a forebrain-specific knockout of Mbp via the Cre/lox system, with a knock-in sequence of Cre being expressed under the Emx1 promoter. As a result, Mbp is knocked out in the cortex and CC of the cKO mice. In addition, another mouse line was generated to achieve a greater extent of cortical dysmyelination. The FoxG1-IRES-Cre:: Mbp flox (FOXMBP) mice show dysmyelination in the cortex and $\mathrm{CC}$ due to a Cre knock-in inder the FoxG1 promoter, since FoxG1 is expressed by neurons and glial cells of the forebrain 4 days prior to Emx1 in the mouse embryo. With this, I aimed to target the knockout of $M b p$, in the forebrain, to a greater extent.

\subsubsection{EMXMBP and FOXMBP show reduced MBP levels in the cortex and CC}

To validate the successful knockout of Mbp in the forebrain of the two cKO mouse lines, I estimated the relative amount of MBP via SDS PAGE and Immunoblotting. In case of the EMXMBP cKO mice I found a reduction in MBP in the WM (consisting of the CC and fimbria) and cortex. However, while this reduction was not statistically significant in the WM (Figure 14 A), it was in the cortex (Figure 14 B). As an internal control for all samples, I included the cerebellum, which should not be affected by the Cre recombinase activity. As expected, the MBP levels in the cerebellum of cKO mice were normal compared to controls (Figure $14 \mathrm{C}$ ). Similarly, in case of the FOXMBP cKO mice, I found a significant reduction in MBP levels in the WM (Figure $20 \mathrm{~A}$ ) but not in the cortex (Figure $20 \mathrm{~B}$ ), with the 


\section{Discussion And Conclusions}

cerebellum also showing comparable levels of MBP (Figure $20 \mathrm{C}$ ). This can be due to human error caused during either tissue dissection or sample preparation. Due to the inherent limitations of the immunoblotting technique furthered by human error, the immunohistochemical characterization of the two mouse lines was paramount. I stained both EMXMBP and FOXMBP mice to visualise the forebrainspecific knockout of Mbp. The representative images for both EMXMBP (Figure $15 \mathrm{~A}$ ) and FOXMBP (Figure $21 \mathrm{~A}$ ) showed a clear reduction in MBP intensities in the cortex and CC of the CKO mice but not in the rest of the brain. I quantified the percentage of area positive for MBP and in case of EMXMBP cKO mice and found a remarkable reduction in both the CC (Figure $15 \mathrm{~B}$ ) and cortex (Figure $15 \mathrm{C}$ ) of the cKO mice compared to controls. However, the percentage of area positive for MBP in the CC of FOXMBP cKO mice was not significantly reduced (Figure 21 B), while the cortex showed a significant reduction in MBP (Figure 21 C). In addition, the striatum, a subcortical structure, of FOXMBP cKO mice also appeared to be dysmyelinated. In part, this can explain the starker reduction in myelin in the CC and cortex of this line compared to the EMXMBP mice. Since the striatum, a region in close proximity to the CC is also dysmyelinated; infiltration of healthy OPCs may be reduced thereby preventing myelination of forebrain-specific regions close by. To estimate MBP intensity in fluorescently labelled slices, the percentage of area positive for MBP is calculated by setting the threshold for the calculated intensity in the ImageJ software. Since the CC of control mice is so heavily myelinated, the intensity threshold may not representative of the true intensity of MBP and results in lowered values. When these are compared to cKO mice, the reduction in MBP is not as apparent as in representative images. With the advent of new techniques such as light sheet fluorescent microscopy (LSFM) (Santi, 2011) and tissue clearing techniques (Ertürk et al., 2012; Pan et al., 2016; Stefaniuk et al., 2016), one can image the entire brain volume instead of quantifying individual slices. This allows one to investigate not only region-specific changes but also axis-dependent gradients and positions of cells within the brain. However, since tissue clearing techniques rely on removing the lipid from the tissue, imaging MBP becomes challenging. Recent development of reflective microscopy, which relies on light deflected by the lipid rich myelinated regions, in combination with LSFM (Wu et al., 


\section{Discussion And Conclusions}

2017) provide promising avenues to visualise the cortical dysmyelination in the two mouse models.

By quantifying MBP levels, using both biochemical and immunohistochemical techniques, I validated the successful knockout of $\mathrm{Mbp}$ from the forebrain of both EMXMBP and FOXMBP cKO mice. Taking into account the limitations both techniques afford, I can say with certainty that both EMXMBP and FOXMBP mice lack MBP, and therefore compact myelin, in the forebrain while the rest of the CNS is normally myelinated.

\subsubsection{EMXMBP and FOXMBP exhibit mild gliosis and have more oligodendrocytes in regions lacking compact myelin}

Although the shiverer mouse model is central to the field of myelin-related research and was discovered nearly 50 years ago, very little focus has been put on investigating the health status of other glial cell types. Nomura and colleagues reported in 2013, an increase in Iba1 positive microglia in the shiverer spinal cord. However, this was not reflected on the transcriptomic level (Nomura et al., 2013). What's fascinating about this mouse model is that despite a severe dysmyelinating phonotype that results in a short lifespan, it shows no signs of axonal degeneration (Griffiths et al., 1998), neuroinflammation (Joshi et al., 2015) and the oligodendrocytes appear to be morphologically normal (Kwiecien et al., 1998).

Since, we generated two mouse models that mimic the shiverer environment in a forebrain-specific manner, it was crucial to investigate the neuropathology in these regions. Moreover, I was curious to see how the shiverer-like environment survives with age, something not documented in the shiverer mice due to their early demise. Thus, 4-month old EMXMBP and FOXMBP mice were assessed for wellestablished markers of gliosis. I observed mild yet significant increase in astrocytes (Figure $16 \mathrm{~A}$ ) and microglia (Figure $16 \mathrm{~B}$ ) in the CC but not in the cortex of EMXMBP cKO mice. Although there was an increase in microglial numbers, they did not seem to be activated (Figure $16 \mathrm{C}$ ). There was a slight increase in Tlymphocyte infiltration in the CC of cKO mice but not in the cortex (Figure 16 D). EMXMBP cKO mice show no axonal degeneration in both the $\mathrm{CC}$ and cortex (Figure $16 \mathrm{E}$ ). In case of the FOXMBP mice, a remarkable increase in astrogliosis 


\section{Discussion And Conclusions}

was seen in both the CC and cortex (Figure 22 A). Moreover, there were more microglia in the CC but not in the cortex (Figure 22 B), and they seemed more phagocytically active in the CC than cortex (Figure $22 \mathrm{C}$ ). The FOXMBP cKO mice, however, showed no signs of T-lymphocyte infiltration in the $\mathrm{CC}$ or the cortex (Figure 22 D). Neither do they show signs of axonal degeneration (Figure 22 E). These data suggest that there is a mild form of gliosis in the CC, for the most part, of both EMXMBP and FOXMBP cKO mice. It is possible that the increase in microglia and astrocytes is a by-product of there being less myelin and hence, more space to accommodate more cells. However, no axonal degeneration is observed at this age in these mice, consistent with data published on shiverer mice (Nomura et al., 2013). The slight increase in the number of T-lymphocytes in the CC of EMXMBP mice may suggest a compromised blood-brain barrier. It appears that even in a prolonged state of dysmyelination, only a mild form of neuropathology is observed in both mouse models. I observed an increase in the number of nuclei within the forebrain, especially the CC of both EMXMBP and FOXMBP mice. Very little is commented upon this in case of shiverer mice with researchers believing this increase is due to an increase in astrogliosis and microgliosis (Tyszka et al., 2006). However, the mild increase in astrocyte and microglia numbers that I observed in my mice, could not account for such a stark increase in the number of nuclei.

To investigate if any of these cells are OPCs or OLs, I stained sections of 6-month old mice for Olig2 and CAII. I counted the density of OPCs and OLs in the CC and cortex. Unfortunately, in case of EMXMBP mice, I could not conduct statistical analysis due to low sample numbers of cKO mice. However, the trend alone suggests a marked increase in the density of OLs in the CC of cKO mice (Figure 17 B). For the FOXMBP mice, I noted a significant increase in OPCs as well as OLs in the CC (Figure 23 B and C). The cortex in both mouse lines showed slightly more numbers of OLS and OPCs but not significantly high (Figure 17 and 23 E and $\mathbf{F}$ ).

One thing of note here was that the Olig2 primary antibody was not very strong as can be seen from the representative images (Figure 17 and 23). This could have caused a gross underestimation the real density of OPCs especially in case of EMXMBP mice. The fact that there is an increase in the OL density within the CC 


\section{Discussion And Conclusions}

raises questions about where so many OLs come from. One hypothesis is that a subpopulation of OLs reside within the grey matter. They are known as satellite OLs. They are believed to quiescent but react to demyelinating events cased due to aging or injury and begin myelinating exposed axons (Szuchet et al., 2011). However, the signals that mediate their transition into myelin-forming OLs is not known. Furthermore, how and whether these OLs migrate from the grey matter into the WM is yet to be elucidated. One other hypothesis of mine, is that the OLs wrapping axons of the CC recruit OPCs in hopes of rescuing the dysmyelination. However, the recruited OLs are also deficient in MBP and cannot successfully myelinate the axons. In addition, in the absence of MBP, the resident OLs don not retract their processes. They continue to attempt myelinating axons even when they are incapable of forming compact myelin. Perhaps, even if healthy OLs can be recruited to the $\mathrm{CC}$ of these mice, they may not necessarily be able to myelinate axons that are still wrapped by the resident OL's processes. Hence they remain nearby waiting to myelinate. At this point, this is pure conjecture as we do not know whether the resident OLs even recruit new OLs and if so, what kind of signalling mechanisms underpin this recruitment. These mice could prove very useful to study these questions in the near future.

Based on the data from both mouse lines, it seems likely that in the absence of compact myelin, there is more area in these regions that can be inhabited by other resident cells such as microglia, astrocytes, OPCs and OLs. However, it does not suggest that maladaptive mechanisms are under way.

\subsubsection{Ultrastructural analysis of EMXMBP and FOXMBP mice shows region-dependent disparity with regards to dysmyelination}

Ultrastructural analysis of shiverer mice shows the extent to which myelin is either loosely packed or completely absent around axons within the entire CNS (Bird et al., 1978; Dentinger et al., 1982; Inoue et al., 1981; Privat et al., 1979). To assess the extent to which the CC is dysmyelinated in my mouse models, EM was conducted on ultrathin sections of the CC of 4-month old EMXMBP and FOXMBP mice. The $\mathrm{rCC}$ of EMXMBP cKO mice showed a dorsal-ventral disparity with respect to dysmyelination with the dorsal CC containing more shiverer-like 


\section{Discussion And Conclusions}

myelination and the ventral CC appeared to be normally myelinated (Figure $18 \mathrm{~A}$ ). The dorsal CC of cKO mice possessed a mixture of myelinated, loosely myelinated and unmyelinated fibres (Figure $18 \mathrm{D}$ ). Interestingly, large calibre axons tended to be more compactly myelinated whereas small calibre axons has shiverer-like myelin. In addition, a larger number of small calibre axons appeared to be loosely myelinated. This suggests that large calibre axons are more likely to be preferentially myelinated as compared to small calibre axons in the EMXMBP cKO mice. This could be, perhaps, due to the capability of large calibre axons to recruit health, non-recombined OLs. The g-ratio analysis of the myelinated axons in both cKO and control mice further confirmed that although large calibre axons are comparably myelinated in both cKO and control mice, the small calibre axons tended to be hypomyelinated (Figure $18 \mathrm{E}$ ). This suggests that healthy OLs may infiltrate the ventral CC, which is closer to the normally myelinated regions such as the striatum and myelinate axons properly. Whereas, the recombined OLs tend to reside in the dorsal $\mathrm{CC}$ and preferentially myelinated small calibre axons. OPCs arising from the dorsal domains express Emx1 in the forebrain (Kessaris et al., 2006). This explains the dorsal-ventral disparity with regards to recombination and hence, dysmyelination observed in the EMXMBP mice. The FOXMBP mice, however, showed a dysmyeliantion disparity along a different axis. In these mice, the $\mathrm{CCC}$ was normally myelinated, almost indistinguishable from that of a control mouse. The rCC, however, was almost entirely dysmyelinated with shiverer-like axons (Figure 24 A). For this reason, only the rCC was compared in both mouse lines. There was a significant reduction in compactly myelinated axons and an increase in the number of axons with shiverer-like myelin (Figure $24 \mathbf{C}$ ). the relative frequency distribution of axons with both compact and shiverer-like myelin was slightly but significantly skewed in the rCC of cKO mice compared to controls (Figure 24 D). G-ratios could not be compared between control and cKO mice because of the absence of compactly myelinated axons in the $\mathrm{rCC}$ of the cKO mice. Since the striatum is also affected in these mice, it stands to argue that the extent of dysmyelination is more pronounced in these mice compared to EMXMBP mice. Moreover, myelination in the forebrain begins caudally and progresses in a rostral fashion (Downes and Mullins, 2014). FoxG1, which is expressed during embryonic development, has been shown to mediate the expansion of the 


\section{Discussion And Conclusions}

telencephalon at the rostral end (Kumamoto and Hanashima, 2017). This also explains why the rCC showed a greater degree of dysmyelination than the $\mathrm{CCC}$ in these mice.

\subsection{What exhibiting sign of catatonia entails for the EMXMBP mice}

To assess the role of cortical myelination in higher brain functions, the EMXMBP mice were exposed to an array of behavioural tests. These tests are commonly used to examine a variety of behavioural domains such as anxiety, social behaviour, motor performance, sensorimotor gating, vision, hearing, spatial memory, and executive function. These experiments were conducted by Sahab Arinrad and Anja Ronnenberg of the Max Planck Institute for Experimental Medicine. I was provided the data for analysis. EMXMBP cKO mice showed no behavioural abnormalities or deficits as summarized in Table 9 and Table 10 except for increased signs of catatonia. Catatonia is a 'psychomotor syndrome' that leads to a temporary disruption in executive function in the absence of any typical motor deficits (Janova et al., 2018). Catatonia as evaluated via the bar test, which consists of placing a mouse with its front paws on a bar and counting the amount of time it takes to withdraw its paws off the bar. The larger the immobility latency, the stronger the signs of catatonia are. Both female and male EMXMBP mice were tested at different ages for signs of catatonia. On average, a wild type mouse spends less than 0.5 seconds immobile on the bar. In case of female cKO mice, they showed significantly higher latencies compare to control mice at the 1-year time-point, whereas male cKO mice started showing higher latencies at 6 months of age. They continued to show progressing signs of catatonia with age until the 11-month time-point. However, both female and male mice tested at 17 months of age (Figure 19). The immobility latencies of individual male cKO mice was highly variable, more so than that of female mice. In their study, Janova and colleagues argue that Cnp deficient mice exhibit higher signs of catatonia due to increased inflammation, evident by increased microgliosis. When the microglia are ablated pharmacologically, they report an amelioration of the catatonic symptom. In case of the EMXMBP mice, since I observed a mild increase in microglia in the CC (Figure 16 B), it could signal an increase in microglia with age and perhaps explain 


\section{Discussion And Conclusions}

the increase in catatonic phenotype in these mice. However, the lack of deficits with regards to other tests for executive function, such as the hurdle test and puzzle box begs to question if the increased catatonia-like signs are truly conducive to an executive dysfunction.

With these data, I conclude that either cortical dysmyelination has a very subtle effect on behaviour or that more behavioural tests need to be conducted that specifically target the output of cortical regions and do not include subcortical stations.

\subsection{Does cortical dysmyelination, which extends into the corpus callosum, affect behavioural lateralization?}

Since the decision to use a paw for retrieval and carrying out the action are mediated by different regions of the cortex, I wondered if improper myelination in the cortex would hinder the successful transfer off information between hemispheres and regions. Moreover, to investigate the specific role of $\mathrm{CC}$ in the establishment of handedness, I needed a mouse model that was not devoid of MBP in the entire CNS, but the forebrain, and more importantly the CC. Hence, I tested 10-week old FOXMBP mice to assess their paw preference. The FOXMBP mice do not show any impairment in lateralization (Figure 25). Further characterization of the mouse line revealed that the $\mathrm{cCC}$ of these mice is normally myelinated (Figure 24). I concluded that perhaps the fact that the $\mathrm{CCC}$ is normally myelinated is sufficient for normal development of behavioural lateralization. Or perhaps, the CC axons alone are sufficient to establish laterality. Since it is estimated that only $30-70 \%$ of the CC is myelinated (Foster et al., 2019; Sturrock, 1980), it is likely that lateralization of handedness can still occur in the absence of myelin. Since cortical myelination is so heterogeneous, the effect of cortical dysmyelination is too subtle when considering a behavioural output in the form of paw laterality. 


\section{Bibliography}

Aggarwal, S., Snaidero, N., Pähler, G., Frey, S., Sánchez, P., Zweckstetter, M., Janshoff, A., Schneider, A., Weil, M.-T., Schaap, I.A.T., et al. (2013). Myelin membrane assembly is driven by a phase transition of myelin basic proteins into a cohesive protein meshwork. PLoS Biol. 11, e1001577.

Allen, N.J., and Barres, B.A. (2009). Neuroscience: Glia - more than just brain glue. Nature 457, 675-677.

Almeida, R.G., and Lyons, D.A. (2017). On myelinated axon plasticity and neuronal circuit formation and function. J. Neurosci. 37, 10023-10034.

Amunts, K. (2010). Structural indices of asymmetry. In The two halves of the brain: information processing in the cerebral hemispheres, $\mathrm{K}$. Hugdahl, and $\mathrm{R}$. Westerhausen, eds. (The MIT Press), pp. 145-176.

Baumann, N., and Pham-Dinh, D. (2001). Biology of oligodendrocyte and myelin in the mammalian central nervous system. Physiol. Rev. 81, 871-927.

Beattie, R., and Hippenmeyer, S. (2017). Mechanisms of radial glia progenitor cell lineage progression. FEBS Lett. 591, 3993-4008.

Bengtsson, S.L., Nagy, Z., Skare, S., Forsman, L., Forssberg, H., and Ullén, F. (2005).

Extensive piano practicing has regionally specific effects on white matter development. Nat. Neurosci. 8, 1148-1150.

Bergles, D.E., and Richardson, W.D. (2015). Oligodendrocyte development and plasticity. Cold Spring Harb. Perspect. Biol. 8, a020453.

Bin, J.M., Leong, S.Y., Bull, S.-J., Antel, J.P., and Kennedy, T.E. (2012). Oligodendrocyte precursor cell transplantation into organotypic cerebellar shiverer slices: a model to study myelination and myelin maintenance. PLoS One 7, e41237.

Bird, T.D., Farrell, D.F., and Sumi, S.M. (1978). Brain lipid composition of the shiverer mouse: (genetic defect in myelin development). J. Neurochem. 31, 387-391.

Boggs, J.M. (2006). Myelin basic protein: a multifunctional protein. Cell Mol. Life Sci. 63, 1945-1961.

Broca, P. (1865). Sur le siège de la faculté du langage articulé. bmsap 6, 377-393. Brooks, J.L., Della Sala, S., and Darling, S. (2014). Representational pseudoneglect: a review. Neuropsychol Rev 24, 148-165. 


\section{Bibliography}

Bulman-Fleming, M.B., Bryden, M.P., and Rogers, T.T. (1997). Mouse paw preference: effects of variations in testing protocol. Behav. Brain Res. 86, 79-87.

Cabeza, R. (2002). Hemispheric asymmetry reduction in older adults: The HAROLD model. Psychol. Aging 17, 85-100.

Carey, D.P., Smith, G., Smith, D.T., Shepherd, J.W., Skriver, J., Ord, L., and Rutland, A. (2001). Footedness in world soccer: an analysis of France' ' '98. J. Sports Sci. 19, 855-864.

Cassells, B., Collins, R.L., and Wahlsten, D. (1990). Path analysis of sex difference, forebrain commissure area and brain size in relation to degree of laterality in selectively bred mice. Brain Res. 529, 50-56.

Chernoff, G. (1981). Shiverer: an autosomal' ' recessive mutant mouse with myelin deficiency .J. Hered.

Choi, E.H., Blasiak, A., Lee, J., and Yang, I.H. (2019). Modulation of neural activity for myelination in the central nervous system. Front. Neurosci. 13, 952.

Collins, R.L. (1968). On the inheritance of handedness. I. Laterality in inbred mice. J. Hered. 59, 9-12.

Collins, R.L. (1969a). On the inheritance of handedness. J. Hered 60, 117-119.

Collins, R.L. (1969b). On the inheritance of handedness. II. Selection for sinistrality in mice. J. Hered. 60, 117-119.

Collins, R.L. (1975). When left-handed mice live in right-handed worlds. Science 187, 181-184.

Corballis, M.C. (2012). Lateralization of the human brain. Prog. Brain Res. 195, 103121.

Coren, S., and Porac, C. (1977). Fifty centuries of right-handedness: the historical record. Science 198, 631-632.

Czopka, T., Ffrench-Constant, C., and Lyons, D.A. (2013). Individual oligodendrocytes have only a few hours in which to generate new myelin sheaths in vivo. Dev. Cell 25, 599-609.

de Ferra, F., Engh, H., Hudson, L., Kamholz, J., Puckett, C., Molineaux, S., and Lazzarini, R.A. (1985). Alternative splicing accounts for the four forms of myelin basic protein. Cell 43, 721-727. 
de Villers-Sidani, E., Chang, E.F., Bao, S., and Merzenich, M.M. (2007). Critical period window for spectral tuning defined in the primary auditory cortex (A1) in the rat. J. Neurosci. 27, 180-189.

Demerens, C., Stankoff, B., Logak, M., Anglade, P., Allinquant, B., Couraud, F., Zalc, B., and Lubetzki, C. (1996). Induction of myelination in the central nervous system by electrical activity. Proc. Natl. Acad. Sci. USA 93, 9887-9892.

Dentinger, M.P., Barron, K.D., and Csiza, C.K. (1982). Ultrastructure of the central nervous system in a myelin deficient rat. J Neurocytol 11, 671-691.

Dere, E., Dahm, L., Lu, D., Hammerschmidt, K., Ju, A., Tantra, M., Kästner, A., Chowdhury, K., and Ehrenreich, H. (2014). Heterozygous ambra1 deficiency in mice: a genetic trait with autism-like behavior restricted to the female gender. Front. Behav. Neurosci. 8, 181.

Downes, N., and Mullins, P. (2014). The development of myelin in the brain of the juvenile rat. Toxicol. Pathol. 42, 913-922.

Emery, B., Agalliu, D., Cahoy, J.D., Watkins, T.A., Dugas, J.C., Mulinyawe, S.B., Ibrahim, A., Ligon, K.L., Rowitch, D.H., and Barres, B.A. (2009). Myelin gene regulatory factor is a critical transcriptional regulator required for CNS myelination. Cell $138,172-$ 185.

Ertürk, A., Becker, K., Jährling, N., Mauch, C.P., Hojer, C.D., Egen, J.G., Hellal, F., Bradke, F., Sheng, M., and Dodt, H.-U. (2012). Three-dimensional imaging of solventcleared organs using 3DISCO. Nat. Protoc. 7, 1983-1995.

Foster, A.Y., Bujalka, H., and Emery, B. (2019). Axoglial interactions in myelin plasticity: Evaluating the relationship between neuronal activity and oligodendrocyte dynamics. Glia 67, 2038-2049.

Francis, N. (2005). Research findings on early first language attrition: implications for the discussion on critical periods in language acquisition. Lang Learn 55, 491-531.

Fünfschilling, U., Supplie, L.M., Mahad, D., Boretius, S., Saab, A.S., Edgar, J., Brinkmann, B.G., Kassmann, C.M., Tzvetanova, I.D., Möbius, W., et al. (2012). Glycolytic oligodendrocytes maintain myelin and long-term axonal integrity. Nature 485, 517-521.

Gazzaniga, M.S. (2000). Cerebral specialization and interhemispheric communication: does the corpus callosum enable the human condition? Brain 123 (Pt 7), 1293-1326. 
Gibson, E.M., Purger, D., Mount, C.W., Goldstein, A.K., Lin, G.L., Wood, L.S., Inema, I., Miller, S.E., Bieri, G., Zuchero, J.B., et al. (2014). Neuronal activity promotes oligodendrogenesis and adaptive myelination in the mammalian brain. Science 344 , 1252304.

Giljov, A., Karenina, K., and Malashichev, Y. (2013). Forelimb preferences in quadrupedal marsupials and their implications for laterality evolution in mammals. BMC Evol. Biol. 13, 61.

Giljov, A., Karenina, K., Ingram, J., and Malashichev, Y. (2015). Parallel emergence of true handedness in the evolution of marsupials and placentals. Curr. Biol. 25, 18781884.

Ginhoux, F., and Prinz, M. (2015). Origin of microglia: current concepts and past controversies. Cold Spring Harb. Perspect. Biol. 7, a020537.

Goodale, M.A. (1988). Hemispheric differences in motor control. Behav. Brain Res. 30, 203-214.

Gordon, J.A., and Stryker, M.P. (1996). Experience-dependent plasticity of binocular responses in the primary visual cortex of the mouse. J. Neurosci. 16, 3274-3286.

Gorski, J.A., Talley, T., Qiu, M., Puelles, L., Rubenstein, J.L.R., and Jones, K.R. (2002). Cortical excitatory neurons and glia, but not GABAergic neurons, are produced in the Emx1-expressing lineage. J. Neurosci. 22, 6309-6314.

Griffiths, I., Klugmann, M., Anderson, T., Yool, D., Thomson, C., Schwab, M.H., Schneider, A., Zimmermann, F., McCulloch, M., Nadon, N., et al. (1998). Axonal swellings and degeneration in mice lacking the major proteolipid of myelin. Science 280, 1610-1613.

Grimshaw, G.M., and Carmel, D. (2014). An asymmetric inhibition model of hemispheric differences in emotional processing. Front. Psychol. 5, 489.

Gruber, D., Waanders, R., Collins, R.L., Wolfer, D.P., and Lipp, H.P. (1991). Weak or missing paw lateralization in a mouse strain (I/LnJ) with congenital absence of the corpus callosum. Behav. Brain Res. 46, 9-16.

Gumpel, M., Baumann, N., Raoul, M., and Jacque, C. (1983). Survival and differentiation of oligodendrocytes from neural tissue transplanted into new-born mouse brain. Neurosci. Lett. 37, 307-311.

Güntürkün, O. (2003). Human behaviour: Adult persistence of head-turning asymmetry. Nature $421,711$. 


\section{Bibliography}

Güntürkün, O., and Ocklenburg, S. (2017). Ontogenesis of Lateralization. Neuron 94, 249-263.

Gyllensten, L., and Malmfors, T. (1963). Myelinization of the optic nerve and its dependence on visual function--a quantitative investigation in mice. J Embryol Exp Morphol 11, 255-266.

Hanashima, C., Li, S.C., Shen, L., Lai, E., and Fishell, G. (2004). Foxg1 suppresses early cortical cell fate. Science 303, 56-59.

Harauz, G., and Boggs, J.M. (2013). Myelin management by the 18.5-kDa and 21.5kDa classic myelin basic protein isoforms. J. Neurochem. 125, 334-361.

Hepper, P.G. (2013). The developmental origins of laterality: fetal handedness. Dev. Psychobiol. 55, 588-595.

Hepper, P.G., Shahidullah, S., and White, R. (1991). Handedness in the human fetus. Neuropsychologia 29, 1107-1111.

Hepper, P.G., Wells, D.L., and Lynch, C. (2005). Prenatal thumb sucking is related to postnatal handedness. Neuropsychologia 43, 313-315.

Holmes, G.L., Milh, M.D.M., and Dulac, O. (2012). Maturation of the human brain and epilepsy. Handb Clin Neurol 107, 135-143.

Hubel, D.H., and Wiesel, T.N. (1970). The period of susceptibility to the physiological effects of unilateral eye closure in kittens. J. Physiol. (Lond.) 206, 419-436.

Hughes, E.G., Orthmann-Murphy, J.L., Langseth, A.J., Bergles, D.E., Hughes, E., Orthmann-Murphy, J., Langseth, A., Bergles, D., Hughes, E., Orthmann-Murphy, J., et al. (2018). Myelin remodeling through experience-dependent oligodendrogenesis in the adult somatosensory cortex. Nat. Neurosci.

Inoue, Y., Nakamura, R., Mikoshiba, K., and Tsukada, Y. (1981). Fine structure of the central myelin sheath in the myelin deficient mutant Shiverer mouse, with special reference to the pattern of myelin formation by oligodendroglia. Brain Res. 219, 8594.

Janova, H., Arinrad, S., Balmuth, E., Mitjans, M., Hertel, J., Habes, M., Bittner, R.A., Pan, H., Goebbels, S., Begemann, M., et al. (2018). Microglia ablation alleviates myelin-associated catatonic signs in mice. J. Clin. Invest. 128, 734-745.

Joshi, D.C., Zhang, C.-L., Lin, T.-M., Gusain, A., Harris, M.G., Tree, E., Yin, Y., Wu, C., Sheng, Z.-H., Dempsey, R.J., et al. (2015). Deletion of mitochondrial anchoring 


\section{Bibliography}

protects dysmyelinating shiverer: implications for progressive MS. J. Neurosci. 35, 5293-5306.

Karlebach, G., and Francks, C. (2015). Lateralization of gene expression in human language cortex. Cortex 67, 30-36.

Katz, L.C. (1999). What's critical for the critical period in visual cortex? Cell 99, 673676.

Kawaguchi, D., Sahara, S., Zembrzycki, A., and O'Leary, D.D.M. (2016). Generation and analysis of an improved Foxg1-IRES-Cre driver mouse line. Dev. Biol. 412, 139147.

Kessaris, N., Fogarty, M., lannarelli, P., Grist, M., Wegner, M., and Richardson, W.D. (2006). Competing waves of oligodendrocytes in the forebrain and postnatal elimination of an embryonic lineage. Nat. Neurosci. 9, 173-179.

Kirschner, D.A., and Ganser, A.L. (1980). Compact myelin exists in the absence of basic protein in the shiverer mutant mouse. Nature 283, 207-210.

Koch, J.D., Miles, D.K., Gilley, J.A., Yang, C.-P., and Kernie, S.G. (2008). Brief exposure to hyperoxia depletes the glial progenitor pool and impairs functional recovery after hypoxic-ischemic brain injury. J. Cereb. Blood Flow Metab. 28, 12941306.

Kral, A. (2013). Auditory critical periods: a review from system's perspective. Neuroscience 247, 117-133.

Kumamoto, T., and Hanashima, C. (2017). Evolutionary conservation and conversion of Foxg1 function in brain development. Dev. Growth Differ. 59, 258-269.

Kwiecien, J.M., O’Connor, L.T., Goetz, B.D., Delaney, K.H., Fletch, A.L., and Duncan, I.D. (1998). Morphological and morphometric studies of the dysmyelinating mutant, the Long Evans shaker rat. J Neurocytol 27, 581-591.

Lasiene, J., Matsui, A., Sawa, Y., Wong, F., and Horner, P.J. (2009). Age-related myelin dynamics revealed by increased oligodendrogenesis and short internodes. Aging Cell 8, 201-213.

Levelt, C.N., and Hübener, M. (2012). Critical-period plasticity in the visual cortex. Annu. Rev. Neurosci. 35, 309-330.

Lo, S.Q., Sng, J.C.G., and Augustine, G.J. (2017). Defining a critical period for inhibitory circuits within the somatosensory cortex. Sci. Rep. 7, 7271. 


\section{Bibliography}

Makinodan, M., Rosen, K.M., Ito, S., and Corfas, G. (2012). A critical period for social experience-dependent oligodendrocyte maturation and myelination. Science 337, 1357-1360.

McGee, A.W., Yang, Y., Fischer, Q.S., Daw, N.W., and Strittmatter, S.M. (2005). Experience-driven plasticity of visual cortex limited by myelin and Nogo receptor. Science 309, 2222-2226.

McKenzie, I.A., Ohayon, D., Li, H., de Faria, J.P., Emery, B., Tohyama, K., and Richardson, W.D. (2014). Motor skill learning requires active central myelination. Science 346, 318-322.

Meschkat, M., Steyer, A.M., Weil, M.-T., Kusch, K., Jahn, O., Piepkorn, L., AgüiGonzalez, P., Ngoc Phan, N.T., Ruhwedel, T., Sadowski, B., et al. (2020). White matter integrity requires continuous myelin synthesis at the inner tongue. BioRxiv.

Micheva, K.D., Wolman, D., Mensh, B.D., Pax, E., Buchanan, J., Smith, S.J., and Bock, D.D. (2016). A large fraction of neocortical myelin ensheathes axons of local inhibitory neurons. Elife 5 .

Molineaux, S.M., Engh, H., de Ferra, F., Hudson, L., and Lazzarini, R.A. (1986). Recombination within the myelin basic protein gene created the dysmyelinating shiverer mouse mutation. Proc. Natl. Acad. Sci. USA 83, 7542-7546.

Nakamura, M., Valerio, P., Bhumika, S., and Barkat, T.R. (2020). Sequential organization of critical periods in the mouse auditory system. Cell Rep. 32, 108070.

Nave, K.-A., and Trapp, B.D. (2008). Axon-glial signaling and the glial support of axon function. Annu. Rev. Neurosci. 31, 535-561.

Nave, K.-A., and Werner, H.B. (2014). Myelination of the nervous system: mechanisms and functions. Annu. Rev. Cell Dev. Biol. 30, 503-533.

Nomura, T., Bando, Y., Bochimoto, H., Koga, D., Watanabe, T., and Yoshida, S. (2013). Three-dimensional ultra-structures of myelin and the axons in the spinal cord: application of SEM with the osmium maceration method to the central nervous system in two mouse models. Neurosci. Res. 75, 190-197.

Pan, C., Cai, R., Quacquarelli, F.P., Ghasemigharagoz, A., Lourbopoulos, A., Matryba, P., Plesnila, N., Dichgans, M., Hellal, F., and Ertürk, A. (2016). Shrinkage-mediated imaging of entire organs and organisms using uDISCO. Nat. Methods 13, 859-867. 


\section{Bibliography}

Papadatou-Pastou, M., Martin, M., Munafò, M.R., and Jones, G.V. (2008). Sex differences in left-handedness: a meta-analysis of 144 studies. Psychol. Bull. 134, 677-699.

Peters, A., and Kemper, T. (2012). A review of the structural alterations in the cerebral hemispheres of the aging rhesus monkey. Neurobiol. Aging 33, 2357-2372.

Popescu, M.V., and Polley, D.B. (2010). Monaural deprivation disrupts development of binaural selectivity in auditory midbrain and cortex. Neuron 65, 718-731.

Preuss, U.W., Meisenzahl, E.M., Frodl, T., Zetzsche, T., Holder, J., Leinsinger, G., Hegerl, U., Hahn, K., and Möller, H.-J. (2002). Handedness and corpus callosum morphology. Psychiatry Res. 116, 33-42.

Privat, A., Jacque, C., Bourre, J.M., Dupouey, P., and Baumann, N. (1979). Absence of the major dense line in myelin of the mutant mouse "shiverer". Neurosci. Lett. 12, 107-112.

Purger, D., Gibson, E.M., and Monje, M. (2016). Myelin plasticity in the central nervous system. Neuropharmacology 110, 563-573.

Raff, M.C., Miller, R.H., and Noble, M. (1983). A glial progenitor cell that develops in vitro into an astrocyte or an oligodendrocyte depending on culture medium. Nature 303, 390-396.

Rakic, P. (2009). Evolution of the neocortex: a perspective from developmental biology. Nat. Rev. Neurosci. 10, 724-735.

Rasband, M.N., and Trimmer, J.S. (2001). Developmental clustering of ion channels at and near the node of Ranvier. Dev. Biol. 236, 5-16.

Roach, A., Takahashi, N., Pravtcheva, D., Ruddle, F., and Hood, L. (1985). Chromosomal mapping of mouse myelin basic protein gene and structure and transcription of the partially deleted gene in shiverer mutant mice. Cell 42, 149-155.

Rosenbluth, J. (1980). Central myelin in the mouse mutant shiverer. J. Comp. Neurol. 194, 639-648.

Saab, A.S., Tzvetavona, I.D., Trevisiol, A., Baltan, S., Dibaj, P., Kusch, K., Möbius, W., Goetze, B., Jahn, H.M., Huang, W., et al. (2016). Oligodendroglial NMDA receptors regulate glucose import and axonal energy metabolism. Neuron 91, 119-132.

Santi, P.A. (2011). Light sheet fluorescence microscopy: a review. J. Histochem. Cytochem. 59, 129-138. 


\section{Bibliography}

Sauer, B. (1987). Functional expression of the cre-lox site-specific recombination system in the yeast Saccharomyces cerevisiae. Mol. Cell. Biol. 7, 2087-2096.

Sauer, B., and Henderson, N. (1988). Site-specific DNA recombination in mammalian cells by the Cre recombinase of bacteriophage P1. Proc. Natl. Acad. Sci. USA 85, 5166-5170.

Sauvageot, C.M., and Stiles, C.D. (2002). Molecular mechanisms controlling cortical gliogenesis. Curr. Opin. Neurobiol. 12, 244-249.

Scharoun, S.M., and Bryden, P.J. (2014). Hand preference, performance abilities, and hand selection in children. Front. Psychol. 5, 82.

Scholz, J., Klein, M.C., Behrens, T.E.J., and Johansen-Berg, H. (2009). Training induces changes in white-matter architecture. Nat. Neurosci. 12, 1370-1371.

Sharma, A., Dorman, M.F., and Spahr, A.J. (2002). Rapid development of cortical auditory evoked potentials after early cochlear implantation. Neuroreport 13, 13651368.

Simeone, A., Gulisano, M., Acampora, D., Stornaiuolo, A., Rambaldi, M., and Boncinelli, E. (1992). Two vertebrate homeobox genes related to the Drosophila empty spiracles gene are expressed in the embryonic cerebral cortex. EMBO J. 11, 25412550.

Simons, M., and Nave, K.-A. (2015). Oligodendrocytes: myelination and axonal support. Cold Spring Harb. Perspect. Biol. 8, a020479.

Sinha, K., Karimi-Abdolrezaee, S., Velumian, A.A., and Fehlings, M.G. (2006). Functional changes in genetically dysmyelinated spinal cord axons of shiverer mice: role of juxtaparanodal Kv1 family K+ channels. J. Neurophysiol. 95, 1683-1695.

Stefaniuk, M., Gualda, E.J., Pawlowska, M., Legutko, D., Matryba, P., Koza, P., Konopka, W., Owczarek, D., Wawrzyniak, M., Loza-Alvarez, P., et al. (2016). Lightsheet microscopy imaging of a whole cleared rat brain with Thy1-GFP transgene. Sci. Rep. 6, 28209.

Stephany, C.-É., Chan, L.L.H., Parivash, S.N., Dorton, H.M., Piechowicz, M., Qiu, S., and McGee, A.W. (2014). Plasticity of binocularity and visual acuity are differentially limited by nogo receptor. J. Neurosci. 34, 11631-11640.

Stephany, C.-É., Ikrar, T., Nguyen, C., Xu, X., and McGee, A.W. (2016). Nogo receptor 1 confines a disinhibitory microcircuit to the critical period in visual cortex. J. Neurosci. $36,11006-11012$. 


\section{Bibliography}

Stiles, J., and Jernigan, T.L. (2010). The basics of brain development. Neuropsychol Rev 20, 327-348.

Sturrock, R.R. (1980). Myelination of the mouse corpus callosum. Neuropathol Appl Neurobiol 6, 415-420.

Suárez, R., Paolino, A., Fenlon, L.R., Morcom, L.R., Kozulin, P., Kurniawan, N.D., and Richards, L.J. (2018). A pan-mammalian map of interhemispheric brain connections predates the evolution of the corpus callosum. Proc. Natl. Acad. Sci. USA 115, 96229627.

Szuchet, S., Nielsen, J.A., Lovas, G., Domowicz, M.S., de Velasco, J.M., Maric, D., and Hudson, L.D. (2011). The genetic signature of perineuronal oligodendrocytes reveals their unique phenotype. Eur. J. Neurosci. 34, 1906-1922.

Tau, G.Z., and Peterson, B.S. (2010). Normal development of brain circuits. Neuropsychopharmacology 35, 147-168.

Tervaniemi, M., and Hugdahl, K. (2003). Lateralization of auditory-cortex functions. Brain Res Brain Res Rev 43, 231-246.

Tomassy, G.S., Berger, D.R., Chen, H.-H., Kasthuri, N., Hayworth, K.J., Vercelli, A., Seung, H.S., Lichtman, J.W., and Arlotta, P. (2014). Distinct profiles of myelin distribution along single axons of pyramidal neurons in the neocortex. Science 344, 319-324.

Tyszka, J.M., Readhead, C., Bearer, E.L., Pautler, R.G., and Jacobs, R.E. (2006). Statistical diffusion tensor histology reveals regional dysmyelination effects in the shiverer mouse mutant. Neuroimage 29, 1058-1065.

van der Knaap, L.J., and van der Ham, I.J.M. (2011). How does the corpus callosum mediate interhemispheric transfer? A review. Behav. Brain Res. 223, 211-221.

Vogel, J.J., Bowers, C.A., and Vogel, D.S. (2003). Cerebral lateralization of spatial abilities: a meta-analysis. Brain Cogn. 52, 197-204.

von Bartheld, C.S., Bahney, J., and Herculano-Houzel, S. (2016). The search for true numbers of neurons and glial cells in the human brain: A review of 150 years of cell counting. J. Comp. Neurol. 524, 3865-3895.

Walz, W. (2000). Role of astrocytes in the clearance of excess extracellular potassium. Neurochem. Int. 36, 291-300. 


\section{Bibliography}

Wright, J., Zhang, G., Yu, T.-S., and Kernie, S.G. (2010). Age-related changes in the oligodendrocyte progenitor pool influence brain remodeling after injury. Dev Neurosci 32, 499-509.

Wu, Y., Kumar, A., Smith, C., Ardiel, E., Chandris, P., Christensen, R., Rey-Suarez, I., Guo, M., Vishwasrao, H.D., Chen, J., et al. (2017). Reflective imaging improves spatiotemporal resolution and collection efficiency in light sheet microscopy. Nat. Commun. 8, 1452.

Xiao, L., Ohayon, D., McKenzie, I.A., Sinclair-Wilson, A., Wright, J.L., Fudge, A.D., Emery, B., Li, H., and Richardson, W.D. (2016). Rapid production of new oligodendrocytes is required in the earliest stages of motor-skill learning. Nat. Neurosci. 19, 1210-1217.

Yandava, B.D., Billinghurst, L.L., and Snyder, E.Y. (1999). Global" cell replacement is feasible via neural stem cell transplantation: evidence from the dysmyelinated shiverer mouse brain. Proc. Natl. Acad. Sci. USA 96, 7029-7034.

Yiu, G., and He, Z. (2006). Glial inhibition of CNS axon regeneration. Nat. Rev. Neurosci. 7, 617-627.

Young, K.M., Psachoulia, K., Tripathi, R.B., Dunn, S.-J., Cossell, L., Attwell, D., Tohyama, K., and Richardson, W.D. (2013). Oligodendrocyte dynamics in the healthy adult CNS: evidence for myelin remodeling. Neuron 77, 873-885.

Zhang, L.I., Bao, S., and Merzenich, M.M. (2001). Persistent and specific influences of early acoustic environments on primary auditory cortex. Nat. Neurosci. 4, 1123-1130. Zhao, Y.-Y., Shi, X.-Y., Qiu, X., Lu, W., Yang, S., Li, C., Chen, L., Zhang, L., Cheng, G.-H., and Tang, Y. (2012). Enriched environment increases the myelinated nerve fibers of aged rat corpus callosum. Anat Rec (Hoboken) 295, 999-1005. 


\section{Appendix}

\subsection{List of Figures}

Figure 1: Schematic diagram showing the development of the brain from a single set of stem cells...

Figure 2: Schematic representation of an oligodendrocyte (OL) myelinating an axonal segment.

Figure 3: The role of MBP in forming compact myelin................................. 6

Figure 4: A timeline of different brain development events in a human............... 8

Figure 5: Evolution of interhemispheric connections. .................................. 11

Figure 6: The modified paw preference paradigm..................................... 32

Figure 7: Establishment of behavioral laterality in young C57BL/6J mice. ......... 45

Figure 8: Lateralized wild-type mice show a U-shaped population distribution of handedness.

Figure 9: Young shiverer mice become lateralized by 9 weeks of age when tested repetitively.

Figure 10: Repetitively tested shiverer mice tend to show more instability with respect to their directionality between tests.

Figure 11: Shiverer mice not tested repetitively during development are still lateralized by 8 weeks of age.

Figure 12: Young shiverer mice not tested repetitively are more stable in the handedness they exhibit.

Figure 13: Schematic showing how the two mouse lines for cortical dysmyelination were generated. 56

Figure 14: Protein estimation of MBP in EMXMBP shows a reduction in protein levels in the forebrain. 58

Figure 15: Immunohistochemical quantification of MBP shows a reduction in EMXMBP mice. 59

Figure 16: EMXMBP mice show a mild form of gliosis at 4 months of age. 62

Figure 17: EMXMBP mice appear to have more OLs in the CC and cortex 64

Figure 18: Ultrastructural analysis of the rCC of EMXMBP mice shows a dorsalventral disparity with respect to the dysmyelination phenotype. 
Figure 19: EMXMBP cKO mice spend more time immobile on the bar in the bar test indicative of a catatonia-like symptom. 68

Figure 20: Protein estimation of MBP in FOXMBP shows a reduction in protein levels in the forebrain. 70

Figure 21: Immunohistochemical quantification of MBP shows a reduction in FOXMBP mice

Figure 22: FOXMBP mice show a mild form of gliosis in the corpus callosum at 4 months of age. 74

Figure 23: FOXMBP mice have more OLs in the CC. 75

Figure 24: The rCC in FOXMBP mice is almost entirely dysmyelinated whereas the $\mathrm{CCC}$ is comparable to control mice. 78

Figure 25: Mice lacking compact myelin in the forebrain do not show impairment of behavioral laterality.

Figure 26: Strength of lateralization at 4 and 8 weeks of age for both weekly and spaced cohorts of shiverer mice.

Figure 27: Strength of lateralization of mice and bipedal and quadrupedal marsupial animals. 84

\subsection{List of Tables}

Table 1: List of commercial assays and kits used 17

Table 2: List of primary antibodies used for immunohistochemistry and western blots.

Table 3: List of secondary antibodies used for immunohistochemistry and western blots. 18

Table 4: Buffers and solutions used for tissue collection.................................. 18

Table 5: Buffers and solutions used for SDS PAGE..................................... 19

Table 6: List of buffers and solutions used for Immunoblot ............................. 20

Table 7: Habituation and retrieval times for the paw preference paradigm ........ 32

Table 8: Summary of different behavioural domains and the corresponding tests conducted to evaluate Emx1Cre/Mbp flox mice ................................................. 35

Table 9: Behavioural analysis of female EMXCre/MBP flox.......................... 108

Table 10: Behavioural analysis of male EMXCre/MBP flox............................. 109 


\subsection{List of Equations}

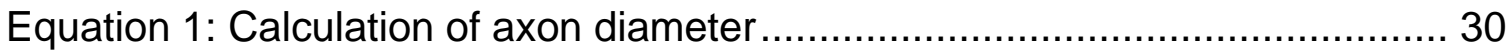

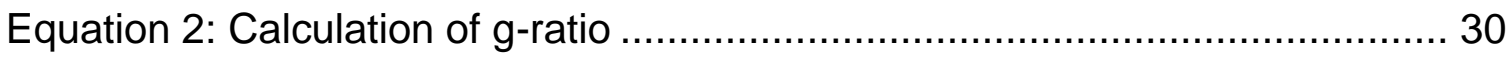

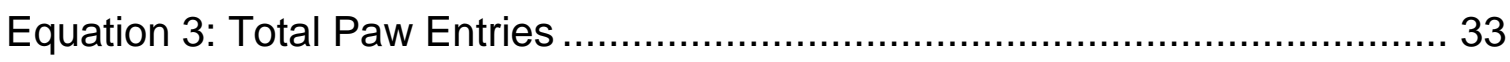

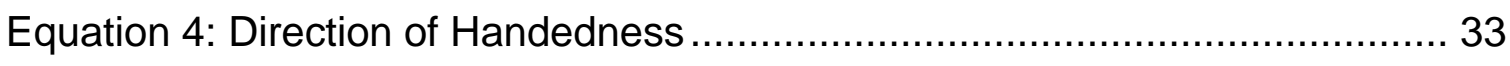

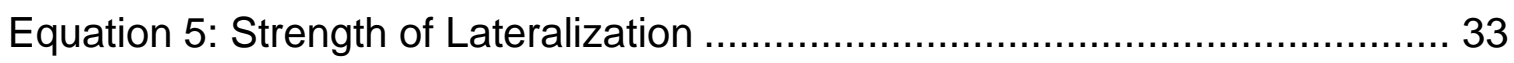

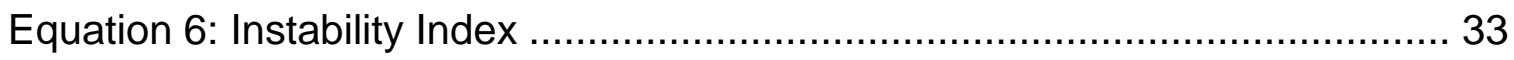

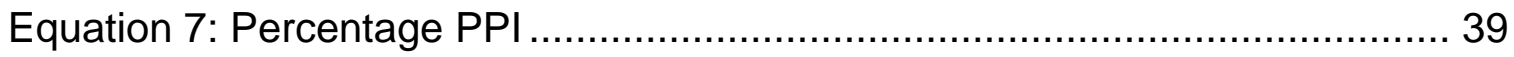

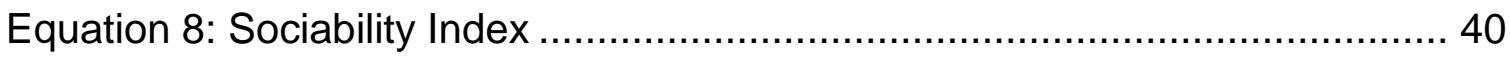

Equation 9: Social Memory Index................................................................... 40

\subsection{List of Abbreviations}

\begin{tabular}{|c|c|}
\hline CNS & Central Nervous System \\
\hline NSCs & Neural Stem Cells \\
\hline VZ & Ventricular Zone \\
\hline NPCs & Neuronal Progenitor Cells \\
\hline RGPs & Radial Glial Progenitors \\
\hline OLs & Oligodendrocytes \\
\hline OPC & Oligodendrocyte Progenitor Cells \\
\hline WM & White Matter \\
\hline Myrf & Myelin Regulatory Factor \\
\hline MBP & Myelin Basic Protein \\
\hline PIP2 & Phosphatidylinositol 4, 5 \\
\hline PNS & Perisphosphate \\
\hline
\end{tabular}




\begin{tabular}{|c|c|}
\hline ODP & Ocular Dominance Plasticity \\
\hline $\mathrm{NgR}$ & Nogo-66 receptor \\
\hline $\mathrm{CC}$ & Corpus Callosum \\
\hline $\mathrm{MRI}$ & Magnetic Resonance Imaging \\
\hline TBST & Tris Buffered Saline-Tween 20 \\
\hline PBS & magnetic resonance imaging \\
\hline PVDF & Polyvinylidene Fluoride \\
\hline PFA & Paraformaldehyde \\
\hline $\mathrm{rCC}$ & rostral Corpus Callosum \\
\hline \#RPE & Right Paw Entries \\
\hline \#LPE & Left Paw Entries \\
\hline \#TPE & Total Paw Entries \\
\hline $\mathrm{PPI}$ & Pre-Pulse Inhibition \\
\hline ANOVA & Analysis of Variance \\
\hline GFAP & Glial Fibrillary Acidic Protein \\
\hline APP & Amyloid Precursor Protein \\
\hline LSFM & Light Sheet Fluorescent Microscopy \\
\hline
\end{tabular}

\subsection{Supplemental Data}


Table 9: Behavioural analysis of female EMXCre/MBP flox.

\begin{tabular}{|c|c|c|c|c|c|c|}
\hline \multirow[b]{3}{*}{ Behavioral paradigms } & \multicolumn{3}{|l|}{ Females } & \multirow{2}{*}{\multicolumn{2}{|c|}{$\mathrm{Em} \mathrm{x}^{\mathrm{Cre}}{ }^{\mathrm{C}} \mathrm{Mbp}^{\mathrm{flox} / \text { flox }}$}} & \multirow[b]{3}{*}{$\mathrm{p}$-value } \\
\hline & \multicolumn{3}{|c|}{ Mbp $^{\text {flox/flox }}$} & & & \\
\hline & Age (months) & $(\mathrm{N})$ & Mean \pm S.E.M. & \multicolumn{2}{|c|}{ 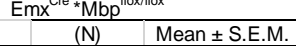 } & \\
\hline \multicolumn{7}{|l|}{ Anxiety - like behavior } \\
\hline Elevated plus maze ( $\%$ time spent in open arm) & 3 & 17 & $30.51 \pm 6.05$ & 11 & $34.91 \pm 8.64$ & 0.67 \\
\hline Elevated plus maze (\% visits to open arm) & 3 & 17 & $21.55 \pm 2.35$ & 11 & $20.00 \pm 2.34$ & 0.66 \\
\hline Open Field (latency to periphery, s) & 3 & 17 & $15.82 \pm 3.09$ & 11 & $9.27 \pm 1.51$ & 0.014 \\
\hline Open Field (visits periphery, \#) & 3 & 17 & $34.41 \pm 3.87$ & 11 & $33.36 \pm 5.93$ & 0.57 \\
\hline Open Field (visits intermediate, \#) & 3 & 17 & $18.29 \pm 1.44$ & 11 & $20.82 \pm 1.81$ & 0.28 \\
\hline Open Field (visits center, \#) & 3 & 17 & $4.65 \pm 0.56$ & 11 & $5.82 \pm 0.63$ & 0.19 \\
\hline Light-Dark Box (latency light, s) & 3 & 17 & $33.59 \pm 9.68$ & 11 & $36.91 \pm 15.26$ & 0.48 \\
\hline Light-Dark Box (visits light, \#) & 3 & 17 & $3.06 \pm 0.70$ & 11 & $7.82 \pm 1.52$ & 0.013 \\
\hline Light-Dark Box (visits dark, \#) & 3 & 17 & $3.47 \pm 0.78$ & 11 & $8.18 \pm 1.35$ & 0.0045 \\
\hline \multicolumn{7}{|l|}{ Motor performance and exploration } \\
\hline Hole board (nose-pokes, \#) & 3 & 17 & $30.12 \pm 3.04$ & 11 & $30.36 \pm 3.78$ & 0.96 \\
\hline & & & & & & \\
\hline Rotarod day 1 (latency to fall, $\mathrm{s}$ ) & 3 & 17 & $98.59 \pm 13.4$ & 11 & $116.8 \pm 17.37$ & 0.41 \\
\hline Rotarod day 2 (latency to fall, s) & 3 & 17 & $125.2 \pm 9.97$ & 11 & $129.7 \pm 22.54$ & 0.84 \\
\hline Rotarod day 1 (latency to fall, s) & 5 & 17 & $201.4 \pm 11.24$ & 11 & $163.7 \pm 16.76$ & 0.06 \\
\hline Rotarod day 2 (latency to fall, s) & 5 & 17 & $211.9 \pm 11.18$ & 11 & $186.6 \pm 21.91$ & 0.27 \\
\hline Grip strength (au) & 3 & 17 & $106.0 \pm 3.09$ & 11 & $101.5 \pm 3.99$ & 0.38 \\
\hline & 5 & 17 & $88.18 \pm 4.39$ & 11 & $11.3 \pm 4.27$ & 0.07 \\
\hline Hearing & & & & & & \\
\hline Acoustic startle at $65 \mathrm{~dB}(\mathrm{au})$ & 4 & 17 & $0.61 \pm 0.05$ & 11 & $0.58 \pm 0.05$ & 0.79 \\
\hline Acoustic startle at $120 \mathrm{~dB}(\mathrm{au})$ & 4 & 17 & $4.1 \pm 0.74$ & 11 & $4.19 \pm 0.96$ & 0.94 \\
\hline Acoustic startle at $65 \mathrm{~dB}(\mathrm{au})$ & 12 & 15 & $2.42 \pm 0.48$ & 11 & $0.74 \pm 0.06$ & 0.0067 \\
\hline Acoustic startle at $120 \mathrm{~dB}(\mathrm{au})$ & 12 & 15 & $2.42 \pm 0.48$ & 11 & $2.16 \pm 0.34$ & 0.68 \\
\hline Acoustic startle at $65 \mathrm{~dB}(\mathrm{au})$ & 19 & 9 & $1.03 \pm 0.15$ & 8 & $0.89 \pm 0.11$ & 0.96 \\
\hline Acoustic startle at $120 \mathrm{~dB}$ (au) & 19 & 9 & $2.48 \pm 0.68$ & 8 & $2.46 \pm 0.51$ & 0.62 \\
\hline & & & & & & \\
\hline Vision & & & & & & \\
\hline Cued platform training day 1 (escape latency, s) & 3 & 17 & $23.15 \pm 2.85$ & 10 & $27.69 \pm 4.80$ & 0.54 \\
\hline Cued platform trianing day 2 (escape latency, s) & 3 & 17 & $9.58 \pm 1.54$ & 10 & $8.35 \pm 1.28$ & 0.90 \\
\hline Sensorimotor gating & & & & & & \\
\hline Pre-pulse inhibition $(\varnothing, \%)$ & 4 & 17 & $26.62 \pm 5.4$ & 11 & $17.96 \pm 5.92$ & 0.30 \\
\hline & 12 & 15 & $26.37 \pm 5.53$ & 11 & $32.61 \pm 6.26$ & 0.46 \\
\hline & 19 & 9 & $-9.01 \pm 13.84$ & 8 & $13.24 \pm 5.17$ & 0.17 \\
\hline Spatial memory and reversal (Morris water maze) & & & & & & \\
\hline Probe: total distance $(\mathrm{cm})$ & 3 & 17 & $1937 \pm 142.5$ & 11 & $2152 \pm 101.1$ & 0.47 \\
\hline Probe: average velocity $(\mathrm{cm} / \mathrm{s})$ & 3 & 17 & $21.53 \pm 1.58$ & 11 & $23.92 \pm 1.12$ & 0.47 \\
\hline Probe: time in target zone (s) & 3 & 17 & $32.35 \pm 3.42$ & 11 & $36.9 \pm 4.07$ & 0.41 \\
\hline Probe: visits to target zone (\#) & 3 & 17 & $10.88 \pm 1.04$ & 11 & $13.00 \pm 1.20$ & 0.20 \\
\hline Probe: latency to target zone (s) & 3 & 17 & $5.46 \pm 1.04$ & 11 & $3.89 \pm 0.92$ & 0.50 \\
\hline Reversal: total distance $(\mathrm{cm})$ & 13 & 17 & $2071 \pm 137.1$ & 11 & $1922 \pm 126.6$ & 0.26 \\
\hline Reversal: average velocity $(\mathrm{cm} / \mathrm{s})$ & 13 & 17 & $23.026 \pm 1.52$ & 11 & $21.36 \pm 1.41$ & 0.26 \\
\hline Reversal: time in target zone (s) & 13 & 17 & $25.49 \pm 1.54$ & 11 & $29.48 \pm 3.79$ & 0.27 \\
\hline Reversal: visits to target zone (\#) & 13 & 17 & $10.82 \pm 0.87$ & 11 & $12.80 \pm 1.65$ & 0.25 \\
\hline Reversal: latency to target zone (s) & 13 & 17 & $2.60 \pm 0.63$ & 11 & $2.56 \pm 0.55$ & 0.85 \\
\hline Catatonic signs & & & & & & \\
\hline Bar test (time immobile on the bar, $s$ ) & 3 & 17 & $0.52 \pm 0.37$ & 11 & $0.18 \pm 0.05$ & 0.64 \\
\hline & 5 & 17 & $0.11 \pm 0.006$ & 11 & $0.12 \pm 0.01$ & 0.54 \\
\hline & 8 & 17 & $0.11 \pm 0.02$ & 11 & $0.13 \pm 0.01$ & 0.27 \\
\hline & 9 & 17 & $0.11 \pm 0.005$ & 11 & $0.13 \pm 0.01$ & 0.37 \\
\hline & 12 & 17 & $0.13 \pm 0.02$ & 11 & $0.26 \pm 0.08$ & 0.022 \\
\hline & 17 & 17 & $0.15 \pm 0.04$ & 11 & $0.53 \pm 0.24$ & 0.07 \\
\hline & & & & & & \\
\hline Executive function & & & & & & \\
\hline Hurdle test trial 1 (ratio, $\mathrm{s} /($ crossings +1$))$ & 3 & 17 & $5.74 \pm 0.64$ & 11 & $6.50 \pm 87$ & 0.40 \\
\hline Hurdle test trial 2 (ratio, $\mathrm{s} /($ crossings +1$))$ & 3 & 17 & $1.70 \pm 0.16$ & 11 & $1.73 \pm 0.13$ & 0.58 \\
\hline Hurdle test trial 1 (ratio, $\mathrm{s} /($ crossings +1$))$ & 8 & 17 & $2.39 \pm 0.16$ & 11 & $4.91 \pm 1.15$ & 0.0123 \\
\hline Hurdle test trial 2 (ratio, $\mathrm{s} /($ crossings +1$))$ & 8 & 17 & $1.48 \pm 0.15$ & 11 & $2.07 \pm 0.37$ & 0.25 \\
\hline Hurdle test trial 1 (ratio, $\mathrm{s} /($ crossings +1$))$ & 13 & 15 & $5.14 \pm 1.38$ & 11 & $10.38 \pm 6.48$ & 0.56 \\
\hline Hurdle test trial 2 (ratio, $\mathrm{s} /($ crossings +1$)$ ) & 13 & 15 & $3.05 \pm 0.41$ & 11 & $2.76 \pm 0.31$ & 0.83 \\
\hline Hurdle test trial 1 (ratio, $\mathrm{s} /($ crossings +1$))$ & 17 & 10 & $8.42 \pm 2.46$ & 9 & $15.54 \pm 10.59$ & 0.60 \\
\hline Hurdle test trial 2 (ratio, $\mathrm{s} /($ crossings +1$)$ ) & 17 & 10 & $4.37 \pm 1.10$ & 9 & $8.23 \pm 5.27$ & 0.72 \\
\hline Hurdle test trial 1 (ratio, $\mathrm{s} /($ crossings +1$))$ & & & & & & \\
\hline Hurdle test trial 2 (ratio, $\mathrm{s} /($ crossings +1$))$ & & & & & & \\
\hline Hurdle test trial 1 (ratio, $\mathrm{s} /($ crossings +1$))$ & & & & & & \\
\hline Hurdle test trial 2 (ratio, $\mathrm{s} /($ crossings +1$))$ & & & & & & \\
\hline & & & & & & \\
\hline Puzzle box ( $\varnothing$ escape latency, s): open & 18 & 10 & $17.65 \pm 2.5$ & 9 & $52.94 \pm 24.79$ & 0.24 \\
\hline Puzzle box (Ø escape latency, s): gateway & 18 & 10 & $9.87 \pm 1.50$ & 9 & $36.62 \pm 25.52$ & 0.78 \\
\hline Puzzle box ( $\varnothing$ escape latency, s): gateway + bedding & 18 & 10 & $58.29 \pm 11.41$ & 9 & $67.04 \pm 17.11$ & 0.96 \\
\hline Puzzle box ( $\varnothing$ escape latency, s): paper tissue & 18 & 10 & $99.18 \pm 15.10$ & 9 & $99.47 \pm 40.45$ & 0.35 \\
\hline Puzzle box ( $\varnothing$ escape latency, $s$ ): nesting material & 18 & 10 & $174.3 \pm 22.23$ & 9 & $151.8 \pm 31.72$ & 0.45 \\
\hline
\end{tabular}




\section{Curriculum vitae}

Table 10: Behavioural analysis of male EMXCre/MBP flox.

\begin{tabular}{|c|c|c|c|c|c|c|c|}
\hline \multirow[b]{3}{*}{ Behavioral paradigms } & \multicolumn{3}{|l|}{ Males } & & \multirow{2}{*}{ flox/filox } & \multirow[b]{3}{*}{ p-value } \\
\hline & \multicolumn{3}{|c|}{ Mbp $p^{\text {flox/flox }}$} & \multicolumn{2}{|c|}{ 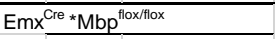 } & & \\
\hline & Age (months) & $(\mathrm{N})$ & Mean \pm S.E.M. & & (N) & Mean \pm S.E.M. & \\
\hline & & & & & & & \\
\hline Anxiety -like behavior & & & & & & & \\
\hline Elevated plus maze (\% time spent in open arm) & 3 & 23 & $16.17 \pm 2.33$ & & 19 & $12.94 \pm 2.03$ & 0.31 \\
\hline Elevated plus maze (\% visits to open arm) & 3 & 23 & $19.22 \pm 1.16$ & & 19 & $16.52 \pm 1.22$ & 0.12 \\
\hline Open Field (latency to periphery, s) & 3 & 23 & $30.57 \pm 8.14$ & & 19 & $23.37 \pm 8.81$ & 0.55 \\
\hline Open Field (visits periphery, \#) & 3 & 23 & $11.35 \pm 1.06$ & & 19 & $10.74 \pm 1.35$ & 0.56 \\
\hline Open Field (visits intermediate, \#) & 3 & 23 & $15.04 \pm 1.27$ & & 19 & $13.63 \pm 1.36$ & 0.45 \\
\hline Open Field (visits center, \#) & 3 & 23 & $4.13 \pm 0.43$ & & 19 & $3.32 \pm 0.42$ & 0.19 \\
\hline Light-Dark Box (latency light, s) & 2 & 23 & $70.10 \pm 14.67$ & & 19 & $58.11 \pm 13.25$ & 0.99 \\
\hline Light-Dark Box (visits light, \#) & 2 & 23 & $5.17 \pm 0.76$ & & 19 & $4.42 \pm 0.78$ & 0.48 \\
\hline Light-Dark Box (visits dark, \#) & 2 & 23 & $5.74 \pm 0.76$ & & 19 & $5 \pm 0.8$ & 0.48 \\
\hline Motor performance and exploration & & & & & & & \\
\hline Hole board (nose-pokes, \#) & 3 & 23 & $21.13 \pm 2.05$ & & 19 & $17.79 \pm 2.45$ & 0.3 \\
\hline & & & & & & & \\
\hline Rotarod day 1 (latency to fall, $s$ ) & 3 & 23 & $136.3 \pm 9.48$ & & 19 & $113.3 \pm 12.58$ & 0.14 \\
\hline Rotarod day 2 (latency to fall, $s$ ) & 3 & 23 & $159.5 \pm 12.66$ & & 19 & $167.1 \pm 15.4$ & 0.70 \\
\hline Rotarod day 1 (latency to fall, $s$ ) & 5 & 23 & $130.7 \pm 10.96$ & & 19 & $142.3 \pm 11.62$ & 0.47 \\
\hline Rotarod day 2 (latency to fall, $s$ ) & 5 & 23 & $159.7 \pm 12.77$ & & 19 & $161.8 \pm 11.33$ & 0.90 \\
\hline Grip strength (au) & 3 & 23 & $98.8 \pm 4.77$ & & 19 & $110.6 \pm 4.35$ & 0.08 \\
\hline & 5 & 23 & $98.28 \pm 3.06$ & & 19 & $102.8 \pm 2.54$ & 0.27 \\
\hline Hearing & & & & & & & \\
\hline Acoustic startle at $65 \mathrm{~dB}(\mathrm{au})$ & 4 & 23 & $0.53 \pm 0.04$ & & 19 & $0.55 \pm 0.05$ & 0.97 \\
\hline Acoustic startle at $120 \mathrm{~dB}(\mathrm{au})$ & 4 & 23 & $5.68 \pm 0.82$ & & 19 & $6.89 \pm 0.96$ & 0.34 \\
\hline Acoustic startle at $65 \mathrm{~dB}(\mathrm{au})$ & 12 & 23 & $0.77 \pm 0.04$ & & 19 & $0.77 \pm 0.04$ & 0.98 \\
\hline Acoustic startle at $120 \mathrm{~dB}(\mathrm{au})$ & 12 & 23 & $6.35 \pm 0.92$ & & 19 & $5.65 \pm 0.80$ & 0.58 \\
\hline Acoustic startle at $65 \mathrm{~dB}(\mathrm{au})$ & 19 & 23 & $1.57 \pm 0.37$ & & 19 & $0.98 \pm 0.12$ & 0.39 \\
\hline Acoustic startle at $120 \mathrm{~dB}(\mathrm{au})$ & 19 & 23 & $4.55 \pm 0.61$ & & 19 & $5.11 \pm 0.85$ & 0.67 \\
\hline & & & & & & & \\
\hline Vision & & & & & & & \\
\hline Cued platform training day 1 (escape latency, s) & 3 & 23 & $22.21 \pm 3.83$ & & 19 & $14.58 \pm 2.41$ & 0.22 \\
\hline Cued platform trianing day 2 (escape latency, $s$ ) & 3 & 23 & $11.98 \pm 2.15$ & & 19 & $8.37 \pm 1.39$ & 0.22 \\
\hline Sensorimotor gating & & & & & & & \\
\hline Pre-pulse inhibition $(\varnothing, \%)$ & 4 & 23 & $37.85 \pm 4.55$ & & 19 & $36.84 \pm 4.51$ & 0.88 \\
\hline & 12 & 23 & $39.71 \pm 4.17$ & & 19 & $35.08 \pm 6.53$ & 0.54 \\
\hline & 19 & 23 & $9.44 \pm 6.25$ & & 18 & $18.15 \pm 7.00$ & 0.36 \\
\hline Spatial memory and reversal (Morris water maze) & & & & & & & \\
\hline Probe: total distance $(\mathrm{cm})$ & 3 & 23 & $2107 \pm 57.33$ & & 19 & $2275 \pm 68.27$ & 0.10 \\
\hline Probe: average velocity $(\mathrm{cm} / \mathrm{s})$ & 3 & 23 & $23.42 \pm 0.64$ & & 19 & $25.29 \pm 0.76$ & 0.10 \\
\hline Probe: time in target zone (s) & 3 & 23 & $32.32 \pm 1.78$ & & 19 & $29.87 \pm 2.06$ & 0.37 \\
\hline Probe: visits to target zone (\#) & 3 & 23 & $13.7 \pm 0.79$ & & 19 & $15.05 \pm 0.71$ & 0.21 \\
\hline Probe: latency to target zone (s) & 3 & 23 & $4.28 \pm 0.74$ & & 19 & $4.19 \pm 0.90$ & 0.64 \\
\hline Reversal: total distance (cm) & 3 & 23 & $1528 \pm 67.92$ & & 19 & $1586 \pm 79.97$ & 0.58 \\
\hline Reversal: average velocity $(\mathrm{cm} / \mathrm{s})$ & 3 & 23 & $16.98 \pm 0.72$ & & 19 & $17.63 \pm 0.89$ & 0.59 \\
\hline Reversal: time in target zone (s) & 3 & 23 & $33.51 \pm 2.25$ & & 19 & $36.64 \pm 2.59$ & 0.36 \\
\hline Reversal: visits to target zone (\#) & 3 & 23 & $14.78 \pm 0.87$ & & 19 & $13.32 \pm 0.73$ & 0.21 \\
\hline Reversal: latency to target zone (s) & 3 & 23 & $3.19 \pm 0.59$ & & 19 & $4.30 \pm 0.72$ & 0.16 \\
\hline Catatonic signs & & & & & & & \\
\hline Bar test (time immobile on the bar, s) & 2 & 23 & $0.13 \pm 0.01$ & & 19 & $0.20 \pm 0.01$ & 0.15 \\
\hline & 5 & 23 & $0.13 \pm 0.02$ & & 19 & $0.15 \pm 0.02$ & 0.16 \\
\hline & 6 & 23 & $0.21 \pm 0.08$ & & 19 & $0.42 \pm 0.11$ & 0.0135 \\
\hline & 7 & 23 & $0.2 \pm 0.07$ & & 19 & $0.47 \pm 0.12$ & 0.0314 \\
\hline & 8 & 22 & $0.27 \pm 0.1$ & & 19 & $0.89 \pm 0.33$ & 0.0027 \\
\hline & 11 & 23 & $0.15 \pm 0.04$ & & 19 & $0.29 \pm 0.07$ & 0.0049 \\
\hline & 17 & 23 & $0.20 \pm 0.07$ & & 18 & $0.32 \pm 0.08$ & 0.09 \\
\hline & & & & & & & \\
\hline Executive function & & & & & & & \\
\hline Hurdle test trial 1 (ratio, $\mathrm{s} /($ crossings +1$)$ ) & 3 & 23 & $4.90 \pm 0.63$ & & 19 & $4.07 \pm 0.32$ & 0.46 \\
\hline Hurdle test trial 2 (ratio, $\mathrm{s} /($ crossings +1$))$ & 3 & 23 & $1.67 \pm 0.11$ & & 19 & $1.52 \pm 0.12$ & 0.37 \\
\hline Hurdle test trial 1 (ratio, $\mathrm{s} /($ crossings +1$))$ & 7 & 23 & $3.43 \pm 0.62$ & & 19 & $2.65 \pm 0.33$ & 0.81 \\
\hline Hurdle test trial 2 (ratio, $\mathrm{s} /($ crossings +1$))$ & 7 & 23 & $1.50 \pm 0.08$ & & 19 & $1.37 \pm 0.09$ & 0.23 \\
\hline Hurdle test trial 1 (ratio, $\mathrm{s} /($ crossings +1$))$ & 8 & 23 & $2.16 \pm 0.32$ & & 19 & $2.30 \pm 0.54$ & 0.80 \\
\hline Hurdle test trial 2 (ratio, $\mathrm{s} /($ crossings +1$))$ & 8 & 23 & $1.55 \pm 0.15$ & & 19 & $1.52 \pm 0.14$ & 0.81 \\
\hline Hurdle test trial 1 (ratio, $\mathrm{s} /($ crossings +1$))$ & 9 & 23 & $9.40 \pm 2.16$ & & 19 & $5.28 \pm 1.21$ & 0.45 \\
\hline Hurdle test trial 2 (ratio, $\mathrm{s} /($ crossings +1$))$ & 9 & 23 & $2.97 \pm 0.71$ & & 19 & $2.80 \pm 0.22$ & 0.057 \\
\hline Hurdle test trial 1 (ratio, $\mathrm{s} /($ crossings +1$))$ & 13 & 23 & $3.57 \pm 0.69$ & & 19 & $6.01 \pm 1.34$ & 0.15 \\
\hline Hurdle test trial 2 (ratio, $\mathrm{s} /($ crossings +1$))$ & 13 & 23 & $2.84 \pm 0.49$ & & 19 & $1.96 \pm 0.20$ & 0.35 \\
\hline Hurdle test trial 1 (ratio, $\mathrm{s} /($ crossings +1$)$ ) & 17 & 23 & $39.29 \pm 15.66$ & & 19 & $8.75 \pm 2.16$ & 0.28 \\
\hline Hurdle test trial 2 (ratio, $s /($ crossings +1$)$ ) & 17 & 23 & $3.06 \pm 0.42$ & & 19 & $2.46 \pm 0.23$ & 0.82 \\
\hline Puzzle box ( $\varnothing$ escape latency, s): open & 18 & 23 & $25.22 \pm 7.46$ & & 18 & $36.18 \pm 13.29$ & 0.91 \\
\hline Puzzle box (Ø escape latency, s): gateway & 18 & 23 & $22.91 \pm 7.09$ & & 18 & $13.75 \pm 1.86$ & 0.59 \\
\hline Puzzle box ( $\varnothing$ escape latency, s): gateway + bedding & 18 & 23 & $69.86 \pm 14.38$ & & 18 & $77.37 \pm 12.41$ & 0.32 \\
\hline Puzzle box ( $\varnothing$ escape latency, s): paper tissue & 18 & 23 & $101.5 \pm 18.32$ & & 18 & $5501 \pm 11.25$ & 0.08 \\
\hline Puzzle box ( $\varnothing$ escape latency, $s$ ): nesting material & 18 & 23 & $186.3 \pm 19.06$ & & 18 & $199.9 \pm 21.3$ & 0.54 \\
\hline
\end{tabular}

